Pacific

Journal of

Mathematics

REPRESENTATION TYPE OF COMMUTATIVE

NOETHERIAN RINGS II: LOCAL TAMENESS

Lee Klingler and Lawrence S. Levy 


\title{
REPRESENTATION TYPE OF COMMUTATIVE NOETHERIAN RINGS II: LOCAL TAMENESS
}

\author{
Lee Klingler and Lawrence S. Levy
}

\begin{abstract}
We describe all isomorphism classes of finitely generated $\Lambda$-modules, where $\Lambda$ is any complete local (commutative noetherian) ring whose category of modules of finite length does not have wild representation type. (There is a possible exception to our results, involving characteristic 2.)
\end{abstract}

\section{Introduction.}

This is the second of a 4-paper series [KL1]-[KL4] whose purpose is to determine all isomorphism classes of finitely generated modules over all commutative noetherian rings that do not have wild representation type. The first two papers of the series consider the complete local case of this problem. If all isomorphism classes of finitely generated modules over a ring $\Lambda$ can be described, we call $\Lambda$ finitely-generated tame.

Let $\Lambda$ be a complete local (commutative, noetherian) ring. In [KL1] we showed that the category of $\Lambda$-modules of finite length has wild representation type unless $\Lambda$ belongs to one of the following quite small classes of rings: (i) a homomorphic image of one of two types of rings of Krull dimension 1 that we call "strictly split Dedekind-like" and "unsplit Dedekind-like," and (ii) a type of artinian ring that we call a "Klein ring." See [KL1, $\S \S 1,2]$ for the definition of "wild representation type" and a very brief introduction to the subject from the point of view of commutative noetherian rings.

The purpose of the present paper is to describe all isomorphism classes of finitely generated modules over the rings of types (i) and (ii) above with a possible exception involving characteristic 2 [see (1.1.3) for the extra hypothesis needed to avoid this exception]. The bulk of the present paper is devoted to establishing finitely-generated tameness of strictly split and unsplit Dedekind-like rings themselves. Extending this to homomorphic images of these rings, and to Klein rings, is then not difficult.

We begin by defining the rings with which the bulk of this paper deals.

Notation 1.1. Throughout this paper, ring means "commutative ring," unless otherwise stated. By a local ring $(\Lambda, \mathfrak{m}, k)$ we mean a (commutative) noetherian local $\operatorname{ring} \Lambda$ with maximal ideal $\mathfrak{m}$ and residue field $k=\Lambda / \mathfrak{m}$. 
We use the abbreviation DVR for discrete valuation ring; we do not consider fields to be DVRs.

Consider a commutative diagram of rings:

$$
\begin{aligned}
& \Lambda \subset \Gamma \quad(\Gamma \text { noetherian }) \\
& \downarrow^{\rho} \rho \\
& k \subset \bar{\Gamma}\left(k \text { a field }, \operatorname{dim}_{k} \bar{\Gamma} \text { finite }\right)
\end{aligned}
$$

where the right-hand map $\rho$ is a surjective ring homomorphism, the lefthand map $\rho$ is the restriction of this map to $\Lambda$, "rad" denotes "Jacobson radical," the conditions listed at the right of the diagram hold, and $\Lambda$ is the pullback of this diagram; that is,

$$
\Lambda=\{x \in \Gamma \mid \rho(x) \in k\} .
$$

In this situation, $\mathfrak{m}=\operatorname{ker}(\rho) \subset \Lambda$, and $\Lambda$ is noetherian. (See Lemma 4.2 for a generalization of this last fact.) Moreover, $\mathfrak{m}=\operatorname{rad}(\Lambda)$, and therefore $(\Lambda, \mathfrak{m}, k)$ is a local ring. The following two specializations of this situation are the principal rings considered in this paper.

We call $(\Lambda, \mathfrak{m}, k)$ an unsplit Dedekind-like ring if $(\Gamma, \mathfrak{m}, \bar{\Gamma})$ is a DVR, $\bar{\Gamma}=F$ is a field such that $\operatorname{dim}_{k}(F)=2$, and:

$$
\text { The 2-dimensional field extension } k \subset F \text { is separable. }
$$

The only reason for including condition (1.1.3) in this definition is that, when it fails, so does our tameness proof, and we do not know whether $\Lambda$ is tame, wild, or neither. However, this exception cannot occur for rings of number-theoretic origin, because finite-dimensional extensions of finite fields are always separable.

For the rest of this paper - when $\Lambda$ is unsplit Dedekind-like — we fix an element $\pi \in \Gamma$ such that $\Gamma \pi=\mathfrak{m}$, and call $\pi$ the standard $\Gamma$-generator of $\mathfrak{m}$.

Returning to diagram (1.1.1), we call $(\Lambda, \mathfrak{m}, k)$ a strictly split Dedekindlike ring if $\Gamma=\Gamma_{1} \oplus \Gamma_{2}$, where $\left(\Gamma_{1}, \mathfrak{m}_{1}, k\right)$ and $\left(\Gamma_{2}, \mathfrak{m}_{2}, k\right)$ are DVRs with the common residue field $k$, and $\mathfrak{m}=\mathfrak{m}_{1} \oplus \mathfrak{m}_{2}$. In this situation, $\bar{\Gamma}=k \oplus k$. We usually identify $k$ with the set of elements $(x, x) \in \bar{\Gamma}=k \oplus k$, and this is the way that we interpret the inclusion $k \subset \bar{\Gamma}$ shown in (1.1.1).

Note that we again have $\operatorname{dim}_{k}(\bar{\Gamma})=2$. For the rest of this paper - when $\Lambda$ is strictly split Dedekind-like — we fix elements $\pi_{i} \in \Gamma_{i}(i=1,2)$ such that $\Gamma_{i} \pi_{i}=\mathfrak{m}_{i}$, and we call $\pi_{i}$ the standard $\Gamma_{i}$-generator of $\mathfrak{m}_{i}$.

In both the unsplit and strictly split case $\Gamma$ is clearly the normalization of $\Lambda$.

Remarks 1.2. (i) Two other types of local Dedekind-like rings are defined in [KL1, Definition 2.5], and will play a role later in this series of papers, when we drop the "complete local" hypothesis: (a) "Nonstrictly split 
Dedekind-like rings." These cannot occur in the complete case [KL1, Definition 2.5] and therefore play no role in the present paper. (b) DVRs are considered to be Dedekind-like in this series of papers, so that "Dedekindlike" generalizes "Dedekind." But this paper has nothing new to say about modules over DVRs, so in this paper DVRs are used only as the starting point for building unsplit and strictly split Dedekind-like rings.

(ii) In the present paper, we do not assume that our unsplit and strictly split Dedekind-like rings $(\Lambda, \mathfrak{m}, k)$ are $\mathfrak{m}$-adically complete. The only property we need, that completeness would provide - the Krull-Schmidt Theorem for finitely generated modules - already holds for these two types of Dedekind-like rings [Lemma 1.3]. Not having to worry about the complications of completions will simplify a few of our proofs.

(iii) For interesting examples of naturally-occurring Dedekind-like rings, see $\S 12$.

(iv) An abstract definition of "Dedekind-like local ring" is given in [KL1, Definition 2.5]. This definition is equivalent to the present one by [KL1, Lemmas 2.14-2.16]. But the present, more constructive definition is better suited to the purposes of the present paper.

Lemma 1.3. The Krull-Schmidt Theorem holds for finitely generated modules over unsplit and strictly split Dedekind-like rings.

Proof. Note that $\Lambda$ has no nonzero nilpotent elements, because $\Lambda$ is contained in the direct sum of one or two integral domains. Therefore the total quotient ring of $\Lambda$ is the localization $\Lambda_{Q}$ that inverts all elements of $\Lambda$ that lie outside of every minimal prime ideal. Also, $\Lambda$ is noetherian, $\Lambda \neq \Gamma$, and the local ring $\Lambda$ is an indecomposable ring. Under these circumstances, a special case of [ $\mathbf{L O}$, Theorem 1.1 and Remark 2.2] is that $\Lambda$ satisfies the Krull-Schmidt Theorem for finitely generated modules if and only if primitive idempotents of $\Gamma$ remain primitive in $\Gamma / \operatorname{rad}(\Gamma)$. This last condition is clearly satisfied by unsplit and strictly split Dedekind-like rings.

Because of the length and complexity of this paper, we now state the contents of each section, and very briefly sketch the main ideas in the proofs leading to our main structure theorems.

$\S 2$ Indecomposable modules, unsplit case. This section is a detailed description of the structure of indecomposable finitely generated $\Lambda$-modules, when $\Lambda$ is unsplit Dedekind-like. The proofs of the theory that yields this structure, together with the analogous structure in the strictly split case, occupy Sections 4-10. In particular, proofs of the specific assertions of $\S 2$ occur in $\S 9$.

$\S 3$ Indecomposable modules, strictly split case. This section is a detailed description of the structure of indecomposable finitely generated $\Lambda$-modules, when $\Lambda$ is strictly split Dedekind-like. Proofs of the specific assertions of the $\S 3$ occur in $\S 10$. 
$\S 4$ Separated covers and almost functorial property. In this section we introduce the basic abstract concept that allows us to deal with module structure over rings that are not necessarily algebras over fields. Let $\Lambda$ be an unsplit or strictly split Dedekind-like ring with normalization $\Gamma$. Since $\Gamma$ is either a DVR or the direct sum of two DVRs, the structure of finitely generated $\Gamma$-modules is well-known. The basic difficulty in dealing with $\Lambda$ modules - a difficulty that does not arise when studying only lattices - is that not all $\Lambda$-modules are contained in $\Gamma$-modules.

To deal with this difficulty, we define a "best approximation" to each finitely generated $\Lambda$-module $M$ by means of a $\Lambda$-submodule $S$ of some finitely generated $\Gamma$-module $X=\Gamma S$, together with a surjective $\Lambda$-homomorphism $S \rightarrow M$. We call this homomorphism $S \rightarrow M$ a "separated cover."

It turns out to be a triviality that separated covers of finitely generated $\Lambda$-modules exist. The main result of this section is that separated covers of $\Lambda$-modules have an "almost functorial" property: Every map $f: M^{\prime} \rightarrow M$ of $\Lambda$-modules can be lifted to a map $\theta: S^{\prime} \rightarrow S$ between their separated covers. Moreover, if $f$ is one-to-one or onto, so is $\theta$. In addition, there is a natural extension of $\theta$ to a $\Gamma$-homomorphism $\theta^{*}: X^{\prime} \rightarrow X$ of the $\Gamma$-modules $X^{\prime}$ and $X$ generated by $S^{\prime}$ and $S$ respectively. Thus we can think of $\theta^{*}$ as the "best approximation" to $f$ by a homomorphism of $\Gamma$-modules.

In this section, $\Lambda$ is a much more general ring than in the rest of the paper. In fact, $\Lambda$ is not necessarily local or commutative. We assume that $\Lambda$ is the pullback of a diagram analogous to (1.1.1), in which all rings are noetherian, $k$ is semisimple artinian, $\Gamma$ and $\bar{\Gamma}$ are finitely generated modules over $\Lambda$ and $k$ respectively, and $\rho$ is a ring homomorphism. We do not assume that $\operatorname{ker} \rho=\operatorname{rad} \Gamma$. (For more detail, see (4.1.1).)

We note that additional hypotheses about commutativity, completeness, or the radical would not make anything easier in this section. Moreover we will need some of the additional generality in the final paper of this series, when we consider the nonlocal situation.

Earlier versions of the results in this section were used to find all finitely generated modules over a class of rings that includes some commutative noetherian rings whose completions at nonsingular maximal ideals are strictly split Dedekind-like $[\mathbf{L} 1, \mathbf{L 2}, \mathbf{L 3}]$, and also includes the integral group ring $\mathbb{Z} G$, where $G$ is a nonabelian group of order $p q$ ( $p, q$ distinct primes) $[\mathbf{K}]$. These earlier versions, however, do not apply to unsplit Dedekind-like rings. But the basic idea of separated covers (called "separated representations" in this earlier work) applies to both nonfinitely generated and finitely generated modules, in this previous work. We do not know whether separated covers of nonfinitely generated modules exist, when $\Lambda$ is unsplit Dedekind-like. See Remarks 4.8.

$\S 5$ Isomorphism as matrix problem, unsplit case. In this section we return to unsplit Dedekind-like rings and use separated covers to reformulate the 
problem of classifying isomorphism classes of finitely generated $\Lambda$-modules as a matrix problem involving $k$ and $F$, the residue fields of $\Lambda$ and its normalization $\Gamma$, respectively. In this matrix problem, each $\Lambda$-module is represented by a $\Gamma$-module and a pair of matrices $(A, B)$ with entries in $F$; and two modules turn out to be isomorphic if and only if they have the same associated $\Gamma$-module and their corresponding matrix pairs can be transformed into each other by means of left multiplication by matrices in $\operatorname{GL}(m, k)$ and right multiplication by matrices in a certain subgroup of $\mathrm{GL}(n, F)$. A precise statement of these matrix operations is the last result of this section.

$\S 6$ Isomorphism as matrix problem, strictly split case. In this section we do the analogous reduction to a matrix problem in the strictly split case. Here - since $\bar{\Gamma}=k \oplus k$, a direct sum of two rings - we deal with matrix 4-tuples $\left(A_{1}, A_{2}, B_{1}, B_{2}\right)$ with entries in the field $k$, instead of pairs as in $\S 5$, and the matrix operations are more complicated. But the matrix problem is easier to solve because all matrix operations involve the single field $k$.

$\S 7$ Solution of matrix problem, strictly split case. In this section we recall the solution of this "strictly split" matrix problem, from [KL0]. The results of $\S 6$ and $\S 7$ were previously obtained in $[\mathbf{L} 1, \mathbf{L 3}]$, so we omit most of the details, concentrating on making the terminology and notation consistent with that of the present paper. But we do note that these theorems can be more cleanly proved by quoting [KL0].

$\S 8$ Solution of matrix problem, unsplit case. In this section we solve the matrix problem in the unsplit case. When $\Lambda$ is a $k$-algebra, we can tensor it by $F$. This converts the $k-F$ matrix problem, in the unsplit case, to the split matrix problem over $F$ alone. It can then be solved by using the main result in the split case - with $F$ in place of $k$. The details of this change of scalars argument use Galois theory, and therefore require our hypothesis that $F$ be separable $k$.

Of course, one of the main purposes of this series of papers is to not require that $\Lambda$ be a $k$-algebra (because we want to deal with rings of algebraic integers). To deal with this more general situation, we take the unsplit $k-F$ matrix problem out of context, allowing us to reinterpret the problem as a problem about modules over $k$-algebras. We then solve the problem by reducing it to the split case, getting exactly the same answer as if $\Lambda$ had been a $k$-algebra. The main results of this section give the answer to this $k-F$ matrix problem.

$\S 9$ Proofs: Indecomposable modules, unsplit case. In this section we use the solution of the unsplit matrix problem, given in $\S 8$, to prove that the structure of indecomposable $\Lambda$-modules is as described in $\S 2$.

$\S 10$ Proofs: Indecomposable modules, split case. In this section we use the solution of the split matrix problem, given in $\S 7$, to prove that the structure of indecomposable $\Lambda$-modules is as described in $\S 3$. As in previous 
sections about the split case, we omit most of the details since these are not new results, and we concentrate on making the terminology and notation consistent with that of the rest of this paper.

$\S 11$ Klein rings and homomorphic images of Dedekind-like rings. In this section we show that, if $\Lambda$ is a Klein ring, then $\Lambda$ is quasi-Frobenius, with a unique minimal ideal modulo which $\Lambda$ becomes a homomorphic image of a strictly split Dedekind-like ring. Thus every module over a Klein ring is the direct sum of a free $\Lambda$-module and a module over a strictly split Dedekindlike ring. It follows immediately that Klein rings are tame.

We also show how to apply the previous results (about modules over Dedekind-like rings) to modules over homomorphic images of Dedekind-like rings. Although these descriptions follow from the previous results in this paper, some work is required, because our description of $\Lambda$-modules involves both $\Lambda$ and $\Gamma$, and translating this description to $\Lambda / I$-modules is complicated by the fact that the ideal $I$ of $\Lambda$ might not be an ideal of $\Gamma$.

$\S 12$ Examples. We review some previously known examples of rings whose completions are Dedekind-like, and establish two new examples: The quadratic order $\mathbb{Z}[\sqrt{n}]$ for every square-free integer $n$, and all subrings of squarefree index in $\mathbb{Z}^{(n)}$, where $n$ is any positive integer. More precisely, we show that all completions of these rings are either DVRs or else unsplit or strictly split Dedekind-like.

$\S 13$ Terminological index. An index of definitions and named theorems.

Remark 1.4 (Typographical error in [L1]). The structure of $\Lambda$-modules of finite length, in the strictly split case, was worked out in $[\mathbf{L 1}]$. Unfortunately, a nonrepeatedness condition was omitted when block cycle indecomposable modules were discussed in the introduction to that paper, [L1, p. 68]. However, the theorem is correctly stated in the paper itself [ $\mathbf{L} \mathbf{1}$, Theorem 8.2 and the preceeding paragraph]. This omission is doubly unfortunate because it was also made in [NR], although subsequently corrected in [NRSB].

Since the rings $\Lambda$ considered in the present paper vary from section to section, each section begins by stating what class of rings $\Lambda$ can represent. When the setting is not left-right symmetric, "module" means "left module" unless otherwise stated.

We extend special thanks to Markus Schmidmeier for pointing out to us a serious oversight in the penultimate version of this paper.

\section{Indecomposable modules, unsplit case.}

In this section we describe (but do not prove) how to construct all indecomposable finitely generated $\Lambda$-modules from indecomposable (necessarily uniserial) finitely generated $\Gamma$-modules, in the unsplit case. We also describe, very briefly, how homomorphisms of $\Lambda$-modules arise from homomorphisms of $\Gamma$-modules. See $\S 9$ for proofs of the main results of this section. 
Notation 2.1. In this section $(\Lambda, \mathfrak{m}, k)$ is unsplit Dedekind-like with normalization $(\Gamma, \mathfrak{m}, F)$. Recall that $\rho$ denotes the natural surjection $\Gamma \rightarrow \Gamma / \mathfrak{m}$ $=F$, and let $\bar{\rho}: \Gamma \rightarrow F$, the conjugate of $\rho$, be the composition of $\rho$ with the nonidentity $k=\Lambda / \mathfrak{m}$-automorphism of the 2-dimensional Galois extension $F$ of $k$. Similarly, for $\alpha \in F$ the notation $\bar{\alpha}$ (the conjugate of $\alpha$ ) denotes the image of $\alpha$ under this automorphism, and for a matrix $A$ over $F, \bar{A}$ denotes the matrix whose entries are the conjugates of those of $A$.

Let $t$ be a positive integer or $\infty$. For each such $t$ let $\rho: \Gamma / \mathfrak{m}^{t} \rightarrow F$ denote the map induced by the map called $\rho$ in the preceeding paragraph. We set $\mathfrak{m}^{\infty}=0$. Thus $\Gamma / \mathfrak{m}^{t}$ is always a uniserial $\Gamma$-module, and has infinite length when $t=\infty$.

Recall that we chose a standard $\Gamma$-generator $\pi$ of $\mathfrak{m}$ in Notation 1.1. When $t$ is finite, note that, once $\pi$ has been chosen, there is a natural $F$ linear isomorphism $\sigma: F \cong \mathfrak{m}^{t-1} / \mathfrak{m}^{t}$ defined by $\sigma(\gamma+\mathfrak{m})=\gamma \pi^{t-1}+\mathfrak{m}^{t}$. We usually regard this map as an identification, in which case we have $\left(\Gamma / \mathfrak{m}^{t}\right) \pi^{t-1}=\mathfrak{m}^{t-1} / \mathfrak{m}^{t}=F$, the standard copy of $F$ in $\Gamma / \mathfrak{m}^{t}$. This copy of $F$ is the $\Gamma$-socle of $\Gamma / \mathfrak{m}^{t}$. The following simple fact will be used many times.

(2.1.1) When $t \neq \infty, \neq 1$, the standard copy of $F$ in $\Gamma / \mathfrak{m}^{t}$ satisfies $\rho(F)=0$.

Notation 2.2 (Diagrams). Our constructions of indecomposable finitely generated $\Lambda$-modules begin with a nonempty direct sum $X$ of nonzero uniserial $\Gamma$-modules and a diagram $\mathcal{D}$ associated with $X$, after which we define a $\Lambda$-module $M(\mathcal{D})$ associated with $\mathcal{D}$. The module $\Gamma$-module $X$ has the form:

$$
X=\left(\Gamma / \mathfrak{m}^{i_{1}}\right)^{(m)} \oplus \Gamma /\left(\mathfrak{m}^{j_{1}}\right)^{(m)} \oplus \ldots \oplus\left(\Gamma / \mathfrak{m}^{i_{d}}\right)^{(m)}\left[\oplus\left(\Gamma / \mathfrak{m}^{j_{d}}\right)^{(m)}\right]
$$

where the superscript $(m)$ denotes "direct sum of $m$ copies of," and where the brackets at the end indicate that, in some cases, the final term is not present. We call $m$ the block size of $\mathcal{D}$ and of $X$. (For examples of the diagrams that we are referring to, see (2.4.1) and (2.6.2).)

We now give the set of rules for forming and interpreting diagrams. Let $X$ be given, as in (2.2.1). Each block of summands $\left(\Gamma / \mathfrak{m}^{t}\right)^{(m)}$ is represented, in our diagrams, by a vertical bar with the length label $t$ written over it, as shown in the diagram (2.2.2). Let $\mathcal{D}_{0}$ denote this diagram.

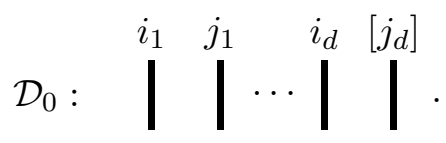

We define $\mathcal{D}$ to be any diagram that can be formed from $\mathcal{D}_{0}$ by a finite number of applications of the following four operations, each of which attaches an "edge" to these vertical bars.

Top-glue. Choose some pair of vertical bars, neither of whose tops has an edge attached to it and connect the tops of these bars by an edge, as shown 
in the two "top-glue" diagrams in (2.2.3). Then label the left or right end of this edge with an invertible $m \times m$ matrix $U$ over $F$, as shown. We view $U$ as being attached to the top of the corresponding vertical bar, as well as to the gluing edge.

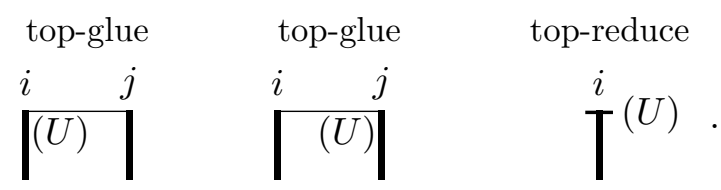

In the case of the first top-glue diagram in (2.2.3), replace the (external) direct sum $\left(\Gamma / \mathfrak{m}^{i}\right)^{(m)} \oplus\left(\Gamma / \mathfrak{m}^{j}\right)^{(m)}$ by the $\Lambda$-submodule given by the following pullback:

$$
\left\{(\boldsymbol{x}, \boldsymbol{y}) \in\left(\Gamma / \mathfrak{m}^{i}\right)^{(m)} \oplus\left(\Gamma / \mathfrak{m}^{j}\right)^{(m)} \mid \rho(\boldsymbol{x})=\bar{\rho}(\boldsymbol{y}) \cdot U\right\} .
$$

(One applies $\rho$ to a tuple by applying $\rho$ to each entry.) The reason for considering $U$ to be attached to the $i$-labeled (rather than $j$-labeled) vertical bar is that, since $U$ is invertible, the set of ordered pairs $(\rho(\boldsymbol{x}), \rho(\boldsymbol{y}))$ that arise from (2.2.4) is the set of all pairs

$$
\left\{(\boldsymbol{\alpha} U, \overline{\boldsymbol{\alpha}}) \in F^{m} \oplus F^{m} \mid \boldsymbol{\alpha} \in F^{m}\right\} .
$$

This will be consistent with our definition of bottom-gluing, below, and with the matrix pairs that will appear when we prove the main theorems of this section.

In the case of the second top-glue diagram, replace the condition $\rho(\boldsymbol{x})=$ $\bar{\rho}(\boldsymbol{y}) \cdot U$ in $(2.2 .4)$ by $\bar{\rho}(\boldsymbol{x}) \cdot U=\rho(\boldsymbol{y})$. The effect of this is to move $U$ to the " $j$ " side of the equal sign in (2.2.5).

If $U=I$ we usually do not explicitly display it.

To explain our view of what has happened in this operation, first note that the pullback in $(2.2 .4)$ contains $\left(\mathfrak{m} / \mathfrak{m}^{i}\right)^{(m)} \oplus\left(\mathfrak{m} / \mathfrak{m}^{j}\right)^{(m)}$; that is, it contains all but the topmost part of $\left(\Gamma / \mathfrak{m}^{i}\right)^{(m)} \oplus\left(\Gamma / \mathfrak{m}^{j}\right)^{(m)}$. We think of the residue module

$$
\frac{\left(\Gamma / \mathfrak{m}^{i}\right)^{(m)} \oplus\left(\Gamma / \mathfrak{m}^{j}\right)^{(m)}}{\left(\mathfrak{m} / \mathfrak{m}^{i}\right)^{(m)} \oplus\left(\mathfrak{m} / \mathfrak{m}^{j}\right)^{(m)}}=F^{(m)} \oplus F^{(m)}
$$

(canonical isomorphism via $\rho$ ) as the "top" of $\left(\Gamma / \mathfrak{m}^{i}\right)^{(m)} \oplus\left(\Gamma / \mathfrak{m}^{j}\right)^{(m)}$.

If $U=I_{m}$, then forming the pullback keeps only half of $F^{(m)} \oplus F^{(m)}$ in (2.2.6), namely the set of ordered pairs of the form $(\boldsymbol{\alpha}, \overline{\boldsymbol{\alpha}})$. A general invertible matrix $U$ "twists" the half of $F^{(m)} \oplus F^{(m)}$ that we are keeping.

Top-reduce. Choose some vertical bar whose top has no attached edge. Say its label is $i$. Attach an edge to this bar - displayed in diagram (2.2.3) by a short thick horizontal line segment - and label the edge with an invertible $m \times m$ matrix $U$ over $F$, as shown. Then replace the corresponding 
summand $\left(\Gamma / \mathfrak{m}^{i}\right)^{(m)}$ of $X$ by its $\Lambda$-submodule

$$
\left\{\boldsymbol{x} \in\left(\Gamma / \mathfrak{m}^{i}\right)^{(m)} \mid \rho(\boldsymbol{x}) \in k^{(m)} U\right\} .
$$

As before, we usually do not display $U$ if $U=I$, and we consider $U$ to be attached to the vertical bar as well as to the reduction edge.

To understand what has happened here, note that the module in (2.2.7) contains the submodule $\left(\mathfrak{m} / \mathfrak{m}^{t}\right)^{(m)}$ of $\left(\Gamma / \mathfrak{m}^{t}\right)^{(m)}$ — all but the top, as before. When $U=I_{m}$ the replacement also keeps the half $k^{(m)}$ of $F^{(m)}=$ $\left(\Gamma / \mathfrak{m}^{t}\right)^{(m)} /\left(\mathfrak{m} / \mathfrak{m}^{t}\right)^{(m)}$, the top of $\left(\Gamma / \mathfrak{m}^{t}\right)^{(m)}$. A general invertible matrix $U$ again "twists" half we are keeping.

Let $S(\mathcal{D})$ be the $\Lambda$-submodule of $X$ that results from whatever top-gluing and top-reductions that have been done. (The various matrix labels $U$ do not need to be the same.) Our discussion of which parts of the original summands of $X$ remain intact by top-gluing and top-reduction shows:

$(2.2 .8) S(\mathcal{D})$ contains the standard copy of $F^{(m)}$ in every summand $\left(\Gamma / \mathfrak{m}^{t}\right)^{(m)}$ of $X$ for which $t \neq \infty, \neq 1$.

Our remaining two operations on diagrams are shown symbolically below. As before we do not usually display $U$ if $U=I$, and we regard $U$ as being attached to the appropriate vertical bar as well as to the gluing or reduction edge.

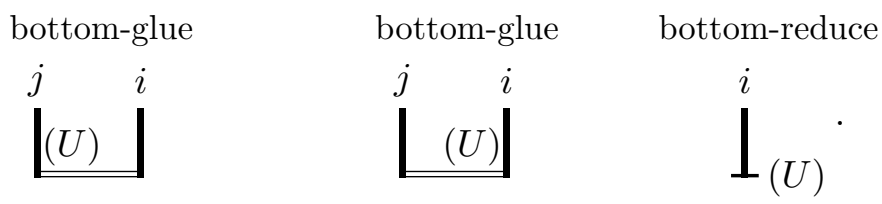

Bottom-glue. Choose some pair of vertical bars whose bottoms have no attached edges and whose length labels $j, i$ are neither $\infty$ nor 1 . Connect the bottoms of these bars with an edge, in the form of an elongated equal sign (as shown), and label the left or right end of this edge with an invertible $m \times m$ matrix $U$ over $F$, as shown in (2.2.9). Since $j \neq \infty$ and $i \neq \infty$, the module $\left(\Gamma / \mathfrak{m}^{j}\right)^{(m)} \oplus\left(\Gamma / \mathfrak{m}^{i}\right)^{(m)}$ contains our standard copy of $F^{(m)} \oplus F^{(m)}$. In the case of the first diagram in (2.2.9), form the $\Lambda$-submodule (2.2.5) of $F^{(m)} \oplus F^{(m)}$, and call this the bottom-gluing module associated with the diagram. In the case of the second diagram in (2.2.9), move $U$ to the " $i$ " side of the comma in (2.2.5), and again call the resulting collection of ordered pairs the bottom-gluing module associated with the diagram.

Bottom-reduce. Choose some vertical bar whose bottom has no attached edge and whose label is neither $\infty$ nor 1 . Attach an edge to the bottom of this bar, displayed as a short thick horizontal line segment, and label this edge with an $m \times m$ invertible matrix $U$ over $F$, as shown. Then form the 
following $\Lambda$-submodule of $F^{(m)}=\left(\mathfrak{m}^{i-1} / \mathfrak{m}^{i}\right)^{(m)}$

$$
k^{(m)} \cdot U
$$

and call it the bottom-reduction module associated with the diagram.

Let $\mathcal{D}$ be the diagram that results from any such bottom and top operations, and let $K(\mathcal{D})$ be the (necessarily direct) sum of the bottom-gluing and the bottom-reduction modules thus formed. Then let

$$
M(\mathcal{D})=S(\mathcal{D}) / K(\mathcal{D})
$$

which we call the $\Lambda$-module associated with $\mathcal{D}$. For (2.2.11) to make sense we need to prove that $K(\mathcal{D}) \subseteq S(\mathcal{D})$, but this follows from (2.2.8).

In order to see what passing modulo $K(\mathcal{D})$ does, first consider the case that every $U$ equals $I_{m}$. Then every bottom-gluing amalgamates one standard copy of $F^{(m)}$ with another; but $\boldsymbol{\alpha}$ in one summand is identified with $-\overline{\boldsymbol{\alpha}}$ in the other. Every bottom-reduction, on the other hand, reduces $S(\mathcal{D})$ modulo the copy of $k^{(m)}$ in the standard copy of $F^{(m)}$. When $U \neq I_{m}$, basically the same thing happens (since $U$ is invertible), but it is twisted by $U$.

The $\Lambda$-modules $M(\mathcal{D})$ thus formed are not always indecomposable. Connectivity of diagram $\mathcal{D}$ is clearly a necessary condition for indecomposability, but is not sufficient.

Reversals. We close these introductory definitions by explicitly noting the effect of moving a matrix label $U$ from one end of a (bottom or top) gluing edge to the other. Top-gluing and bottom-gluing — with $U$ attached to the left-hand end of the edge - involve submodules of $F^{(m)} \oplus F^{(m)}$ consisting of all elements of the form $(\boldsymbol{\alpha} U, \overline{\boldsymbol{\alpha}})$, which can be rewritten in the form $\left(\boldsymbol{\alpha} U,(\overline{\boldsymbol{\alpha}} \bar{U}) \bar{U}^{-1}\right)=\left(\boldsymbol{\beta}, \overline{\boldsymbol{\beta}} \bar{U}^{-1}\right)$. We shall use this in the following form:

(2.2.12) Let $U$ be the matrix label attached to one end of a gluing edge in $\mathcal{D}$. Then moving $U$ to the opposite end of that edge and then replacing $U$ by $\bar{U}^{-1}$ leaves $M(\mathcal{D})$ (not just its isomorphism class!) unchanged.

Notation 2.3 (Sequence manipulation). As in Notation 8.6, let $\mu$ be the mirror image permutation, the permutation that reverses the order of a finite sequence. Thus $\mu\left\{i_{1}, i_{2}, \ldots, i_{d}\right\}=\left\{i_{d}, \ldots, i_{2}, i_{1}\right\}$. Let $\nu$ be the unit forward rotation defined by $\nu\left\{i_{1}, i_{2}, \ldots, i_{d}\right\}=\left\{i_{2}, i_{3}, \ldots, i_{d}, i_{1}\right\}$. A cycle is any cyclic permutation of the form $\nu^{t}$, where $t$ is an integer. If $I$ and $J$ are finite sequences, we let $\{I, J\}$ denote the concatenation of $I$ and $J$, that is, the sequence consisting of the terms of $I$ followed by those of $J$. Thus $\{I, \mu(J)\}$ denotes $I$ followed by the mirror image of $J$, and we have $\mu(\{I, \mu(J)\})=\{J, \mu(I)\}$.

The definitions, below, of our seven "standard diagrams" all make use of the pair of label sequences in decomposition (2.2.1):

$$
I=\left\{i_{1}, i_{2}, \ldots, i_{d}\right\} \quad J=\left\{j_{1}, j_{2}, \ldots, j_{d-1},\left[j_{d}\right]\right\} .
$$


Thus every $i_{\nu}$ and $j_{\nu}$ is a positive integer or $\infty$, and the brackets around $j_{d}$ indicate that $j_{d}$ sometimes does not occur.

We now define some connected diagrams whose associated $\Lambda$-modules turn out to be indecomposable. We reserve the term standard diagram for those diagrams defined in 2.4 and 2.6.

Definitions 2.4 (Standard diagrams, block size 1). The three standard diagrams in this series occur only with block size 1 . The matrix labels $U$ are all one-by-one identity matrices, and are therefore not displayed. (Thus, we must learn from context that the block size is 1.)

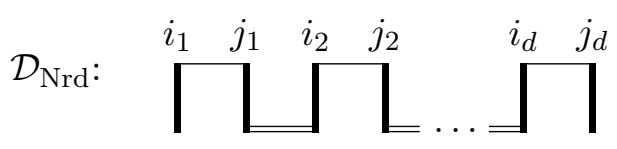

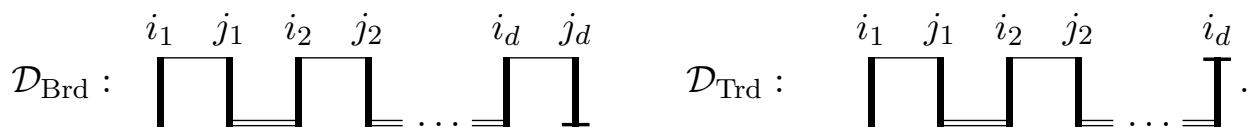

We now complete these definitions.

Nonreduced diagram, $\mathcal{D}_{\mathrm{Nrd}}$. Here the following conditions must be satisfied.

(2.4.2) (i) The block size is $m=1$.

(ii) Only length labels $i_{1}$ and $j_{d}$ can equal $\infty$ or 1 .

(iii) The pair of label sequences $I$ and $J$ must be unsymmetrical in the sense that $J \neq \mu(I)$.

Since the one-by-one identity matrix is its own conjugate inverse, the following is an immediate consequence of (2.2.12):

(2.4.3) Replacing the pair of label sequences $I$ and $J$ in diagram $\mathcal{D}_{\mathrm{Nrd}}$ by $\mu(J)$ and $\mu(I)$, respectively - that is, drawing the left-right mirror image of the diagram - does not change the isomorphism class of $M\left(\mathcal{D}_{\mathrm{Nrd}}\right)$.

In the extreme case $d=1$, no bottom-gluing occurs. When condition (2.4.2)(iii) fails, $M\left(\mathcal{D}_{\mathrm{Nrd}}\right)$ becomes the direct sum of two indecomposable modules [Proposition 9.5].

Bottom-reduced and top-reduced diagram, $\mathcal{D}_{\mathrm{Brd}}$ and $\mathcal{D}_{\mathrm{Trd}}$. The restrictions here are:

(i) The block size is $m=1$.

(ii) Only length label $i_{1}$ can equal $\infty$ or 1 .

Note that $j_{d}$ does not occur in the top-reduced diagram $\mathcal{D}_{\text {Trd }}$. In the extreme case $d=1, \mathcal{D}_{\text {Trd }}$ consists of a single top-reduced vertical bar.

Before proceeding, we note that there are other possibilites for diagrams with block size 1. Some of these [diagrams (2.6.2)] yield indecomposable 
modules in all block sizes, as we shall see, while others are not standard diagrams, even if they are connected. (See, for example, (2.5.1) and Remark 2.10.)

Examples 2.5. The first two diagrams in (2.5.1) are the standard diagrams whose associated $\Lambda$-modules are $\Lambda$ and its residue field $k$, respectively.

$$
\Lambda: \quad \coprod^{\infty} \quad k: \prod^{1} \quad k(\text { illegal }):\left.\right|^{1}
$$

The third diagram in (2.5.1) is illegal because the definition does not allow bottom reduction on a vertical bar with length-label 1. Disregarding this for a moment, note that the $\Lambda$-module associated with a single vertical bar with length-label 1 is $\Gamma / \mathfrak{m}=F$, which is isomorphic to $k \oplus k$ as a $\Lambda$-module. Thus either top-reducing this module or bottom-reducing this module will result in a $\Lambda$-module isomorphic to $k$. Allowing only one of these to be standard associates a unique standard diagram with the $\Lambda$-module $k$. Moreover, this choice is made in such a way that Theorem 2.11 is true for the $\Lambda$-module $k$. We have not investigated whether or not the theorem is true with respect to the second diagram for $k$.

Caution: As just mentioned, the $\Lambda$-module corresponding to single vertical bar with length label 1 and no top or bottom reduction is $\Gamma / \mathfrak{m}=F$. But this is not the standard diagram for $\Gamma / \mathfrak{m}$, because it is not among the diagrams displayed in (2.4.1) and (2.6.2). The standard diagram for $\Gamma / \mathfrak{m}$ is displayed in (2.10.1). Again, the reason for this seeming peculiarity is that Theorem 2.11 holds for this choice of diagrams.

Definitions 2.6 (Standard diagrams, arbitrary block size). The remaining four standard diagrams occur with arbitrary block size. Before stating details, we mention some important differences between these and the standard diagrams of block size 1. (i) None of the length labels is $\infty$ or 1 ; in particular, the associated $\Lambda$-modules $M(\mathcal{D})$ all have finite length. (ii) Both the top and bottom of every vertical bar is either glued or reduced. (iii) The additional conditions that guarantee indecomposability are more complicated. (iv) Each diagram explicitly displays one invertible $m \times m$ matrix label, $U$ or $U^{-1}$ over $F$, which we call the blocking matrix of the diagram. The reason for sometimes using $U$ and sometimes $U^{-1}$ is to achieve uniformity in the uniqueness formulas in Theorem 2.8.

According to our convention, the remaining matrix labels are identity matrices, and are not displayed explicitly. The placement of the blocking matrix in the diagram is somewhat arbitrary. (See Proposition 2.9 about moving $U$.)

We require the following definition. 
(2.6.1) A finite sequence $W$ is repetition-free if there is no strictly shorter sequence $W^{\prime}$ such that $W$ consists of repetitions of $W^{\prime}$.

The following are the standard diagrams associated with arbitrary block size.

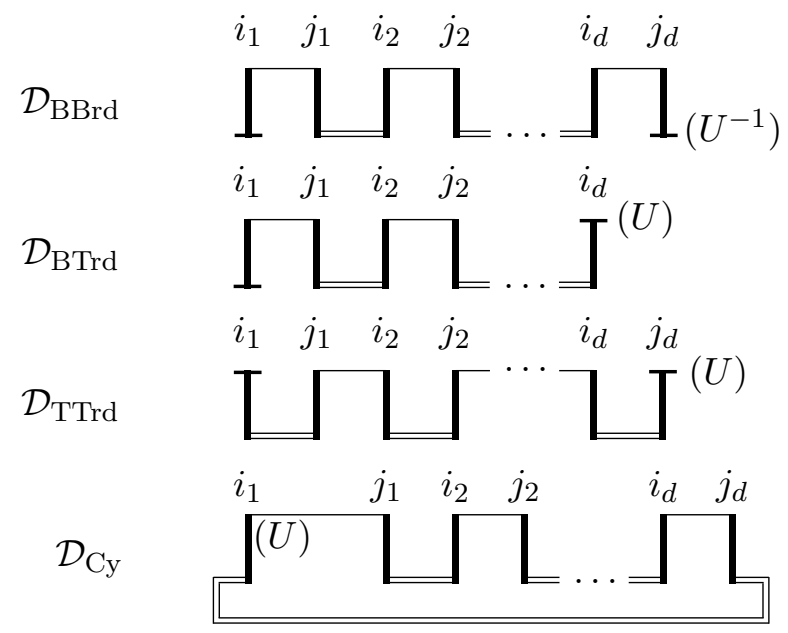

Bottom-bottom-reduced diagrams, $\mathcal{D}_{\mathrm{BBrd}}$. We require the following conditions:

(2.6.3) (i) $U \bar{U}^{-1}$ is indecomposable under similarity.

(ii) None of the length labels is $\infty$ or 1 .

(iii) The concatenated sequence $\{I, \mu(J)\}$ is repetition-free [see (2.6.1)]. Although it is not obvious, for any positive integer $n$, there exist $n \times n$ matrices $U$ satisfying condition (i). (See Remark 2.12.) Note that, when $d=1$, no bottom-gluing occurs.

Bottom-top-reduced diagrams, $\mathcal{D}_{\mathrm{BTrd}}$. We require conditions (2.6.3). Note that $j_{d}$ does not occur in this diagram. When $d=1$ the diagram becomes a single vertical bar, reduced both at the bottom and the top.

Top-top-reduced diagrams, $\mathcal{D}_{\mathrm{TT} \text { Trd }}$. We require conditions (2.6.3) here, too. When $d=1$ no top-gluing occurs.

Cycle diagrams, $\mathcal{D}_{\mathrm{Cy}}$. We require:

(2.6.4) (i) The blocking matrix $U$ is indecomposable under similarity.

(ii) Either the blocking matrix $U$ is not similar to $\bar{U}^{-1}$ or, for all cycles $\nu^{t}, J \neq \nu^{t} \mu(I)$.

(iii) None of the length labels is $\infty$ or 1 .

(iv) The sequence of pairs $\left\{\left(i_{1}, j_{1}\right), \ldots,\left(i_{d}, j_{d}\right)\right\}$ is repetition-free.

When condition (ii) is not satisfied, the resulting module becomes the direct sum of two indecomposable modules [Proposition 9.5]. Unlike the previous types, there is nothing exceptional about the case $d=1$. 
In all four types, unless the repetition-freeness condition holds, the module might be decomposable, and uniqueness of $\mathcal{D}$ can fail [Remark 9.7].

For square matrices $U, V$ over $F$ we write $U \sim V$ for " $U$ is similar to $V$."

Theorem 2.7. Every indecomposable finitely generated $\Lambda$-module is isomorphic to $M(\mathcal{D})$ where $\mathcal{D}$ is one of the standard diagrams in (2.4.1) or (2.6.2), and all such modules are indecomposable. Moreover, every indecomposable finitely generated $\Lambda$-module of infinite length is isomorphic to $M(\mathcal{D})$ for one of the standard diagrams $\mathcal{D}$ in (2.4.1).

Theorem 2.8. Let $\mathcal{D}$ and $\mathcal{D}^{\prime}$ be standard diagrams, with pairs of label sequences $I$ and $J$, and $I^{\prime}$ and $J^{\prime}$ respectively. If these diagrams have blocking matrices, call them $U$ or $U^{-1}$, and $V$ or $V^{-1}$ respectively, and assume that the blocking matrices are located as shown in Diagram (2.6.2).

Then $M(\mathcal{D}) \cong M\left(\mathcal{D}^{\prime}\right)$ if and only if $\mathcal{D}$ and $\mathcal{D}^{\prime}$ are of the same type (i)-(vii) below, and the conditions listed for their type hold.

(i) (Nonreduced) Either $I^{\prime}=I$ and $J^{\prime}=J$, or $I^{\prime}=\mu(J)$ and $J^{\prime}=\mu(I)$.

(ii) (Bottom reduced) $I^{\prime}=I$ and $J^{\prime}=J$.

(iii) (Top reduced) $I^{\prime}=I$ and $J^{\prime}=J$.

(iv) (Bottom-bottom reduced) Either $I^{\prime}=I$ and $J^{\prime}=J$, or $I^{\prime}=\mu(J)$ and $J^{\prime}=\mu(I) ;$ and $V \bar{V}^{-1} \sim U \bar{U}^{-1}$.

(v) (Bottom-top reduced) $I^{\prime}=I$ and $J^{\prime}=J$, and $V \bar{V}^{-1} \sim U \bar{U}^{-1}$.

(vi) (Top-top reduced) Either $I^{\prime}=I$ and $J^{\prime}=J$, or $I^{\prime}=\mu(J)$ and $J^{\prime}=$ $\mu(I)$; and $V \bar{V}^{-1} \sim U \bar{U}^{-1}$.

(vii) (Cycle) Either $I^{\prime}=\nu^{t}(I)$ and $J^{\prime}=\nu^{t}(J)$ (for some cyclic permutation $\nu^{t}$ ) and $V \sim U$; or $I^{\prime}=\nu^{t} \mu(J)$ and $J^{\prime}=\nu^{t} \mu(I)$ (for some cyclic permutation $\nu^{t}$ ) and $V \sim \bar{U}^{-1}$.

We note that, in every case where the pair of label sequences is not uniquely determined by the isomorphism class of $M(\mathcal{D})$, the alternative pair corresponds to the geometric left-right or rotational symmetry of the diagram. However (according to the statement of the theorem), when applying this symmetry to the bars, edges, and length-labels, one leaves the location of the blocking matrix unchanged, in the position shown in standard diagrams (2.6.2).

As mentioned earlier, the placement of the blocking matrix in standard diagrams (2.6.2) is somewhat arbitrary. The precise rules for moving the blocking matrix - and appropriately modifying it - are the subject of our next result.

Proposition 2.9 (Moving $U$ ). Let $\mathcal{D}$ be any standard diagram in the "arbitrary block size" family (2.6.2), except that the blocking matrix $U$ is located at an arbitrary position in the diagram. 
Then each of the following operations on $U$ leaves the isomorphism class of $M(\mathcal{D})$ unchanged.

(i) Move $U$ from one end (left or right) of any gluing edge to the other end of that edge, replacing it with $\bar{U}^{-1}$.

(ii) Move $U$ from one end (top or bottom) of any vertical bar to the other end, replacing it with $U^{-1}$.

In addition, if $\mathcal{D}$ is one of the standard diagrams with a pair of reduction edges, $U$ can be moved unchanged from the top of any vertical bar to the top of any other vertical bar (or from bottom to bottom) without changing the isomorphism class of $M(\mathcal{D})$.

For example, in the first of the diagrams in (2.6.2) - diagram $\mathcal{D}_{\mathrm{BBrd}}-$ we can move $U^{-1}$ to the top of its attached vertical bar, replacing it with $U$. The resulting diagram is now nonstandard, although the $\Lambda$-module that it represents is unchanged.

Remark 2.10 (Efficient vs. standard diagrams). Our standard diagrams handle some indecomposable $\Lambda$-modules inefficiently. This includes all of the indecomposable finitely generated $\Gamma$-modules. One might expect the standard diagram for $\Gamma / \mathfrak{m}^{j}(1 \leq j \leq \infty)$ to be the second diagram in (2.10.1), but it is the first diagram that is standard.

\section{(2.10.1) $\Gamma / \mathfrak{m}^{j}:$}

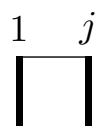

(standard)

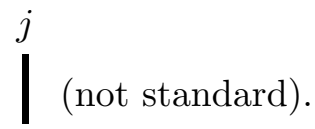

To see that the second diagram is not standard, it suffices to note that it does not appear among the three standard "block size one" diagrams in (2.4.1). (Set $d=1$, the smallest value of $d$ that makes sense in each of these diagrams.) The first diagram in (2.10.1) is a nonreduced diagram; to see that it actually describes $\Gamma / \mathfrak{m}^{j}$ is a simple verification using the definition (2.2.4) of top-gluing.

To see the general principle involved, first note that the only places that the label 1 can occur in a standard diagram are the left-hand end of the three diagrams in (2.4.1) [the "block size 1 only" diagrams], and the right-hand end of the first of these. It is then easy to see the following.

(2.10.2) If a vertical bar with length label 1 occurs in a standard diagram $\mathcal{D}$ in (2.4.1) with an attached top-gluing edge, then the diagram $\mathcal{D}^{\prime}$ that results from deleting the vertical bar and attached edge satisfies $M\left(\mathcal{D}^{\prime}\right) \cong M(\mathcal{D})$. But $\mathcal{D}^{\prime}$ is not a standard diagram.

Making these deletions gives what we call the efficient representation of the module involved, as opposed to the "standard" representation of that module.

If the only purpose of this paper were to describe finitely generated $\Lambda$ modules, we would use the efficient representation. A minor disadvantage of 
this would be that we would have to increase the number of types of standard diagrams by including, for example, diagrams whose left-most gluing edge is a bottom-gluing edge. But this minor disadvantage would be more than offset by the fact that not having an irrelevant vertical bar makes $M\left(\mathcal{D}^{\prime}\right)$ easier to visualize.

The real advantage of keeping the "irrelevant" edge has to do with our Theorem 2.11 about morphisms in $\bmod -\Lambda$ : We do not know whether the theorem applies when standard representations are replaced by efficient ones.

Note that if $\mathcal{D}$ is the disjoint union of diagrams $\mathcal{D}_{1}, \ldots, \mathcal{D}_{m}$ then $M(\mathcal{D})=$ $M\left(\mathcal{D}_{1}\right) \oplus \ldots \oplus M\left(\mathcal{D}_{m}\right)$. Therefore (by the theorems stated so far in this section) every finitely generated $\Lambda$-module is isomorphic to $M(\mathcal{D})$ where $\mathcal{D}$ is a disjoint union of standard diagrams. We can now state our main theorem on morphisms in mod- $\Lambda$, which shows that all such morphisms arise from homomorphisms of modules over the DVR $\Gamma$.

Theorem 2.11 ( $\Lambda$-homomorphisms versus $\Gamma$-homomorphisms). Let $\mathcal{D}^{\prime}$ and $\mathcal{D}$ be disjoint unions of standard diagrams, and let $f: M\left(\mathcal{D}^{\prime}\right) \rightarrow M(\mathcal{D})$ be a $\Lambda$-homomorphism. Then there is a $\Gamma$-homomorphism $f^{* *}: X\left(\mathcal{D}^{\prime}\right) \rightarrow X(\mathcal{D})$ whose restriction $f^{*}$ to $S\left(\mathcal{D}^{\prime}\right)$ is a $\Lambda$-homomorphism: $S\left(\mathcal{D}^{\prime}\right) \rightarrow S(\mathcal{D})$ that takes $K\left(\mathcal{D}^{\prime}\right) \rightarrow K(\mathcal{D})$ and induces $f$. Moreover, if $f$ is one-to-one or onto, then so is any such $f^{*}$.

As we shall see in the proof of this theorem, the natural surjection $S(\mathcal{D}) \rightarrow$ $M(\mathcal{D})$ is a separated cover of $M(\mathcal{D})$, that is, our "best approximation" to $M(\mathcal{D})$ by a $\Lambda$-submodule of some $\Gamma$-module. See $\S 4$ for properties of separated covers.

Remark 2.12 (Indecomposability of $U \bar{U}^{-1}$ ). Condition (2.6.3)(i) on the indecomposability of $U \bar{U}^{-1}$ under similarity, and Condition (2.6.4)(ii) that $U$ not be similar to $\bar{U}^{-1}$, fit in with Theorem 2.8 in a particularly interesting way.

Let $U$ be an invertible $m \times m$ matrix over $F$, and suppose that $U$ is indecomposable under similarity. Thus $U$ can be the companion matrix of any power of any irreducible polynomial (other than $x$ ) in $F[x]$.

By (2.6.4)(ii), $U$ can be the blocking matrix of a cycle diagram $\mathcal{D}_{\mathrm{Cy}}$ in (2.6.2) except if $U \sim \bar{U}^{-1}$ and the label sequences $\left\{i_{\nu}\right\}$ and $\left\{j_{\nu}\right\}$ fail to satisfy a certain nonsymmetry condition. When these restrictions are satisfied, it is the similarity class of $U$ that is an isomorphism invariant of $M\left(\mathcal{D}_{\mathrm{Cy}}\right)$. However it is not obvious what similarity classes are excluded here.

A "Hilbert Theorem 90" for matrices, due to Ballantine [see Lemma 8.11], states that a matrix satisfies the condition $U \sim \bar{U}^{-1}$ if and only if there is a matrix $V$ such that $V \bar{V}^{-1}=U$. According to Condition (2.6.3)(i), such matrices $V$ are precisely the matrices that are allowed to be blocking matrices for the remaining three diagrams that have blocking matrices [see (2.6.2)]. 
Moreover, according to our structure Theorem 2.8, it is the similarity class of $V \bar{V}^{-1}=U$ that is an isomorphism invariant of $M(\mathcal{D})$ in these cases precisely the similarity classes that are excluded from $M\left(\mathcal{D}_{\mathrm{Cy}}\right)$ !

To see what similarity classes of matrices we are talking about, let $U=$ $C(f)$ be the companion matrix of a polynomial $f \in F[x]$, where $f=x^{m}+$ $a_{m-1} x^{m-1}+\cdots+a_{0}$. Since $U$ is invertible in this discussion, we have $a_{0} \neq 0$. Let $g$ be the monic polynomial $g=\left(x^{m} / a_{0}\right) f(1 / x)$. Then it follows easily, with the help of the Cayley-Hamilton Theorem, that $U^{-1} \sim C(g)$, and hence

$$
U \sim \bar{U}^{-1} \Longleftrightarrow f=\left(x^{m} / \bar{a}_{0}\right) \bar{f}(1 / x) .
$$

Using this, it is easy to see that for any positive integer $m$, there exist $m \times m$ matrices $U$ satisfying conditions (2.12.1). For example, use $f(x)=(x+1)^{m}$.

\section{Indecomposable modules, strictly split case.}

In this section we describe (but do not prove) how to construct all indecomposable finitely generated $\Lambda$-modules from indecomposable (necessarily uniserial) finitely generated $\Gamma$-modules, in the strictly split case. This is similar to - but simpler than - the unsplit case. This structure was determined previously, in $[\mathbf{L} 1, \mathbf{L} 3]$. Therefore our focus, in this short section, is to make the terminology of that description consistent with the other terminology of the present paper. This will be especially important when we deal with the nonlocal situation, in the fourth paper of this series. We also describe, very briefly, how homomorphisms of $\Lambda$-modules arise from homomorphisms of $\Gamma$-modules. For proofs, see $\S 10$.

Notation 3.1. Throughout this section $(\Lambda, \mathfrak{m}, k)$ is a strictly split Dedekindlike ring, as in Notation 1.1. Thus the normalization of $\Lambda$ is $\Gamma=\Gamma_{1} \oplus \Gamma_{2}$ where each $\left(\Gamma_{\nu}, \mathfrak{m}_{\nu}, k\right)$ is a DVR and $\mathfrak{m}=\mathfrak{m}_{1} \oplus \mathfrak{m}_{2}$. Following the style of Notation 2.1, we denote by $\rho$ not only the map $\Gamma \rightarrow \bar{\Gamma}=\Gamma / \mathfrak{m}=k \oplus k$ in pullback diagram (1.1.1), but also the maps $\Gamma_{\nu} / \mathfrak{m}_{\nu}^{t} \rightarrow k$ induced by the original map $\rho$. We also use $\rho$ for direct sums of such maps. Moreover, we let $\mathfrak{m}_{\nu}^{\infty}=0$, so that $\Gamma_{\nu}=\Gamma_{\nu} / \mathfrak{m}_{\nu}^{\infty}$.

Recall, from Notation 1.1, that we have chosen a standard $\Gamma_{\nu}$-generator $\pi_{\nu}$ of the maximal ideal $\mathfrak{m}_{\nu}$ of each $\Gamma_{\nu}$.

When $t$ is finite, our choice of generators $\pi_{\nu}$ yields natural $k$-linear isomorphisms $\sigma: k \cong \mathfrak{m}_{\nu}^{t-1} / \mathfrak{m}_{\nu}^{t}$ defined by $\sigma\left(\gamma_{\nu}+\mathfrak{m}_{\nu}\right)=\gamma_{\nu} \pi_{\nu}^{t-1}+\mathfrak{m}_{\nu}^{t}$. We usually regard this map as an identification, in which case we have $\left(\Gamma_{\nu} / \mathfrak{m}_{\nu}^{t}\right) \pi_{\nu}^{t-1}=$ $\mathfrak{m}_{\nu}^{t-1} / \mathfrak{m}_{\nu}^{t}=k$, the standard copy of $k$ in $\Gamma_{\nu} / \mathfrak{m}_{\nu}^{t}$. This copy of $k$ is the $\Gamma_{\nu}$-socle of $\Gamma_{\nu} / \mathfrak{m}_{\nu}^{t}$. The following simple fact will be used many times.

(3.1.1) When $t \neq \infty, \neq 1$, the standard copy of $k$ in $\Gamma_{\nu} / \mathfrak{m}_{\nu}^{t}$ satisfies $\rho(k)=0$. 
Notation 3.2 (Diagrams). As in the unsplit case, our constructions of indecomposable finitely generated $\Lambda$-modules begin with a nonempty direct sum $X$ of nonzero uniserial $\Gamma$-modules and a diagram $\mathcal{D}$ associated with $X$, after which we define a $\Lambda$-module $M(\mathcal{D})$ associated with $\mathcal{D}$. The module $\Gamma$-module $X$ has the form:

$$
\begin{gathered}
X=X_{1} \oplus X_{2} \quad \text { where } \\
X_{1}=\left(\Gamma_{1} / \mathfrak{m}_{1}^{i_{1}}\right)^{(m)} \oplus \ldots \oplus\left(\Gamma_{1} / \mathfrak{m}_{1}^{i_{d}}\right)^{(m)} \\
X_{2}=\left(\Gamma_{2} / \mathfrak{m}_{2}^{j_{1}}\right)^{(m)} \oplus \ldots \oplus\left(\Gamma_{2} / \mathfrak{m}_{2}^{j_{d}}\right)^{(m)} .
\end{gathered}
$$

Here each $i_{\nu}$ and $j_{\nu}$ is a positive integer or $\infty$, and the exponent $(m)$ denotes "direct sum of $m$ copies of." Note that the same number md of indecomposable summands occurs in both $X_{1}$ and $X_{2}$ in (3.2.1). We call $m$ the block size of $\mathcal{D}$ and of $X$. For examples of diagrams, see (3.3.1). We now give the set of rules for forming and interpreting diagrams.

Let $X$ be given, as in (3.2.1). Each block of summands $\left(\Gamma_{\nu} / \mathfrak{m}_{\nu}^{t}\right)^{(m)}$ is represented, in our diagrams, by a vertical bar with a length label $t$ written over it and the index $\nu$ written below it, as shown in (3.2.2). Let $\mathcal{D}_{0}$ denote this diagram.

$$
\mathcal{D}_{0}:\left.\left.\left.\left.\right|_{1} ^{i_{1}}\right|_{2} ^{j_{1}} \cdots\right|_{1} ^{i_{d}}\right|_{2} ^{j_{d}}
$$

We define $\mathcal{D}$ to be any diagram that can be formed from $\mathcal{D}_{0}$ by a finite number of applications of the following two operations, each of which attaches an "edge" to a pair of vertical bars.

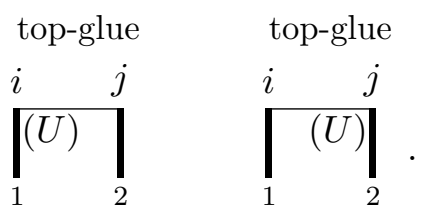

Top-glue. Choose a vertical bar with a 1 below it, and a vertical bar with a 2 below it, and suppose that neither of the tops of these bars has an edge attached; then connect the tops of these bars by an edge, as shown in (3.2.3). Then label the left or right end of this edge with an invertible $m \times m$ matrix $U$ over $k$, as shown. We view $U$ as being attached to the top of the corresponding vertical bar, as well as to the gluing edge.

In the case of the first top-glue diagram in (3.2.3), replace the (external) direct sum $\left(\Gamma_{1} / \mathfrak{m}_{1}^{i}\right)^{(m)} \oplus\left(\Gamma_{2} / \mathfrak{m}_{2}^{j}\right)^{(m)}$ by the $\Lambda$-submodule given by the 
following pullback.

$$
\left\{(\boldsymbol{x}, \boldsymbol{y}) \in\left(\Gamma_{1} / \mathfrak{m}_{1}^{i}\right)^{(m)} \oplus\left(\Gamma_{2} / \mathfrak{m}_{2}^{j}\right)^{(m)} \mid \rho(\boldsymbol{x})=\rho(\boldsymbol{y}) \cdot U\right\} .
$$

(One applies $\rho$ to a tuple by applying $\rho$ to each entry.) The reason for considering $U$ to be attached to the $i$-labeled (rather than $j$-labeled) vertical bar is that, since $U$ is invertible, the set of ordered pairs $(\rho(\boldsymbol{x}), \rho(\boldsymbol{y}))$ that arise from (3.2.4) is the set of all pairs

$$
\left\{(\boldsymbol{\alpha} U, \boldsymbol{\alpha}) \in k^{(m)} \oplus k^{(m)} \mid \boldsymbol{\alpha} \in k^{(m)}\right\} .
$$

This will be consistent with our definition of bottom-gluing, below, and with the matrix pairs that will appear when we prove the main theorems of this section.

In the case of the second top-glue diagram, replace the condition $\rho(\boldsymbol{x})=$ $\rho(\boldsymbol{y}) \cdot U$ in (3.2.4) by $\rho(\boldsymbol{x}) \cdot U=\rho(\boldsymbol{y})$. The effect of this is to move $U$ to the $j$-side of the equal sign in (3.2.5).

We note that, if $U=I$, we get the same module whether we attach $I$ to the $i$-side or the $j$-side of the top-gluing edge. In this case we usually do not explicitly display the matrix $I$.

To explain our view of what has happened in this operation, first note that the pullback in (3.2.4) contains $\left(\mathfrak{m}_{1} / \mathfrak{m}_{1}^{i}\right)^{(m)} \oplus\left(\mathfrak{m}_{2} / \mathfrak{m}_{2}^{j}\right)^{(m)}$; that is, it contains all but the topmost part of $\left(\Gamma_{1} / \mathfrak{m}_{1}^{i}\right)^{(m)} \oplus\left(\Gamma_{2} / \mathfrak{m}_{2}^{j}\right)^{(m)}$. We think of the residue module

$$
\frac{\left(\Gamma_{1} / \mathfrak{m}_{1}^{i}\right)^{(m)} \oplus\left(\Gamma_{2} / \mathfrak{m}_{2}^{j}\right)^{(m)}}{\left(\mathfrak{m}_{1} / \mathfrak{m}_{1}^{i}\right)^{(m)} \oplus\left(\mathfrak{m}_{2} / \mathfrak{m}_{2}^{j}\right)^{(m)}}=k^{(m)} \oplus k^{(m)}
$$

(canonical isomorphism via $\rho$ ) as the "top" of $\left(\Gamma_{1} / \mathfrak{m}_{1}^{i}\right)^{(m)} \oplus\left(\Gamma_{2} / \mathfrak{m}_{2}^{j}\right)^{(m)}$.

If $U=I_{m}$, then forming the pullback keeps only half of $k^{(m)} \oplus k^{(m)}$ in (3.2.6), namely the set of ordered pairs of the form $(\boldsymbol{\alpha}, \boldsymbol{\alpha})$. A general invertible matrix $U$ "twists" the half of $k^{(m)} \oplus k^{(m)}$ that we are keeping.

Let $S(\mathcal{D})$ be the $\Lambda$-submodule of $X$ that results from whatever top-gluing operations that have been done. (The various matrix labels $U$ do not need to be the same.) Our discussion of which parts of the original summands of $X$ remain intact by top-gluing shows:

(3.2.7) $S(\mathcal{D})$ contains the standard copy of $k^{(m)}$ in every summand $\left(\Gamma_{\nu} / \mathfrak{m}_{\nu}^{t}\right)^{(m)}$ of $X$ for which $t \neq \infty, \neq 1$.

Our remaining operations on diagrams are shown symbolically below. As before we do not usually display $U$ if $U=I$, and we regard $U$ as being attached to the appropriate vertical bar as well as to the bottom-gluing edge. 


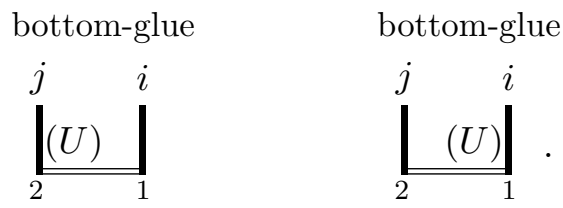

Bottom-glue. Choose a vertical bar with 1 below it and a vertical bar with 2 below it, neither with length label 1 or $\infty$, and without any edge attached to the bottom of either of them. Connect the bottoms of these bars with an edge, in the form of an elongated equal sign (as shown), and label the left or right end of this edge with an invertible $m \times m$ matrix $U$ over $k$, as shown in (3.2.8). Since neither length label is infinite, $\left(\Gamma_{2} / \mathfrak{m}_{2}^{j}\right)^{(m)} \oplus\left(\Gamma_{1} / \mathfrak{m}_{1}^{i}\right)^{(m)}$ contains our standard copy of $k^{(m)} \oplus k^{(m)}$. In the case of the first diagram in (3.2.8), form the $\Lambda$-submodule (3.2.5) of $k^{(m)} \oplus k^{(m)}$, and call it the bottom-gluing module associated with the diagram. In the case of the second diagram in (3.2.8), move $U$ to the $i$-side of the comma in (3.2.5), and again call the resulting set of ordered pairs the bottom-gluing module associated with the diagram.

Let $\mathcal{D}$ be the diagram that results from any such bottom- and top-gluing operations, and let $K(\mathcal{D})$ be the (necessarily direct) sum of the bottomgluing modules thus formed. Then let

$$
M(\mathcal{D})=S(\mathcal{D}) / K(\mathcal{D})
$$

which we call the $\Lambda$-module associated with $\mathcal{D}$. For (3.2.9) to make sense we need to prove that $K(\mathcal{D}) \subseteq S(\mathcal{D})$, and this follows from (3.2.7).

In order to see what passing modulo $K(\mathcal{D})$ does, first consider the case that every $U$ equals $I_{m}$. Then every bottom-gluing amalgamates one standard copy of $k^{(m)}$ with another. When $U \neq I_{m}$, basically the same thing happens (since $U$ is invertible), but it is twisted by $U$.

The $\Lambda$-modules $M(\mathcal{D})$ thus formed are not always indecomposable. Connectivity of diagram $\mathcal{D}$ is clearly a necessary condition for indecomposability, but is not sufficient.

We now define the two types of connected diagrams whose associated $\Lambda$-modules turn out to be indecomposable. We reserve the term standard diagrams for the diagrams defined in 3.3. These definitions make use of the pair of label sequences in decomposition (3.2.1):

$$
I=\left\{i_{1}, i_{2}, \ldots, i_{d}\right\} \quad J=\left\{j_{1}, j_{2}, \ldots, j_{d}\right\} .
$$

Thus every $i_{\nu}$ and $j_{\nu}$ is a positive integer or $\infty$.

Definitions 3.3 (Standard diagrams). Our first standard diagram - a "deleted cycle" diagram - occurs only with block size 1. For deleted cycle diagrams, the matrix labels $U$ are all one-by-one identity matrices and are therefore not displayed. The second diagram — a "block cycle" diagram 
- can occur with arbitrary block size. Each block cycle diagram explicitly displays only one invertible $m \times m$ matrix label $U$ over $k$, called the blocking matrix of the diagram, while all remaining matrix labels (not displayed) are $m \times m$ identity matrices. The placement of the blocking matrix in the diagram is somewhat arbitrary. (See Proposition 3.6 about moving $U$.)
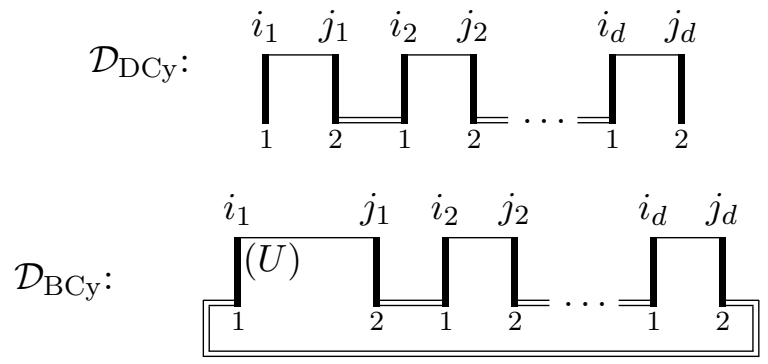

We now complete these definitions.

Deleted cycle diagrams, $\mathcal{D}_{\mathrm{DCy}}$. The following conditions must be satisfied.

(3.3.2) (i) The block size is $m=1$.

(ii) Only labels $i_{1}$ and $j_{d}$ can equal $\infty$ or 1 .

Block cycle diagrams, $\mathcal{D}_{\mathrm{BCy}}$. The following conditions must be satisfied.

(3.3.3) (i) The blocking matrix $U$ is indecomposable under similarity.

(ii) Length labels $\infty$ and 1 cannot occur.

(iii) The sequence of pairs $\left\{\left(i_{1}, j_{1}\right), \ldots,\left(i_{d}, j_{d}\right)\right\}$ is repetition-free. (Recall, from (2.6.1), that a finite sequence $W$ is repetition-free if there is no strictly shorter sequence $W^{\prime}$ such that $W$ consists of repetitions of $W^{\prime}$.) When condition (iii) is not satisfied, a larger block size can be used, and the module $M(\mathcal{D})$ might be decomposable.

Examples 3.4. The first two diagrams below are the standard diagrams whose associated $\Lambda$-modules are $\Lambda$ and $k$, respectively.
$\Lambda: \prod_{1}^{\infty}$
$k: \prod_{1}^{1} 1$
$k$ (nonstandard $):\left.\right|_{1} ^{1}$
also: $\left.\right|_{2} ^{1}$.

The third and fourth diagrams above are nonstandard diagrams whose associated $\Lambda$-modules are again $k$. These are the "efficient" diagrams for $k$, as discussed in Remark 3.7.

Let $I, J$ be as in (3.2.10); and let $I^{\prime}, J^{\prime}$ be another pair of sequences, each again of length $d$. We say that $I, J$ and $I^{\prime}, J^{\prime}$ are equal modulo simultaneous cyclic permutations if there is an integer $c$ such that $I^{\prime}=\nu^{c}(I)$ and $J^{\prime}=$ $\nu^{c}(J)$. (See Notation 2.3.) 
Theorem 3.5. If $\Lambda$ is strictly split Dedekind-like, then every indecomposable finitely generated $\Lambda$-module is isomorphic to $M(\mathcal{D})$ for some deleted cycle or block cycle diagram $\mathcal{D}$, and all such modules are indecomposable.

Let $\mathcal{D}, \mathcal{D}^{\prime}$ be diagrams of these types. (If either diagram has a blocking matrix, assume that this blocking matrix is located as shown in standard diagram (3.3.1).) Then $M(\mathcal{D}) \cong M\left(\mathcal{D}^{\prime}\right)$ if and only if one of the following holds.

(i) Both $\mathcal{D}$ and $\mathcal{D}^{\prime}$ are deleted cycle diagrams, and $\mathcal{D}=\mathcal{D}^{\prime}$, (that is, they have the same label sequences).

(ii) Both $\mathcal{D}$ and $\mathcal{D}^{\prime}$ are block cycle diagrams, they have the same pairs of label seqeunces modulo simultaneous cyclic permutations, and their blocking matrices are similar.

The placement of the blocking matrix in block cycle diagrams is somewhat arbitrary. The precise rules for moving the blocking matrix are the subject of our next result.

Proposition 3.6 (Moving $U$ ). Let $\mathcal{D}$ be any block cycle diagram, except that the blocking matrix $U$ is located at an arbitrary position in the diagram. Then each of the following operations on $\mathcal{D}$ leaves the isomorphism class of $M(\mathcal{D})$ unchanged.

(i) Move $U$ from one end (left or right) of any gluing edge to the other end of that edge, replacing it with $U^{-1}$.

(ii) Move $U$ from one end (top or bottom) of any vertical bar to the other end, replacing it with $U^{-1}$.

Remark 3.7 (Efficient vs. standard diagrams). The discussion given in Remark 2.10 applies here also, with only one very minor change: The indecomposable $\Gamma$-modules are now $\Gamma_{\nu} / \mathfrak{m}_{\nu}^{t}$, rather than the modules $\Gamma / \mathfrak{m}^{t}$ displayed in (2.10.1). Thus, the standard and efficient diagrams for (say) the $\Lambda$-modules $\Gamma_{1} / \mathfrak{m}_{1}^{i}$ are, respectively, the first and second diagrams displayed below.

$$
\Gamma_{1} / \mathfrak{m}_{1}^{i} \text { (standard) : } \prod_{1}^{i} \prod_{2}^{1} \quad \Gamma_{1} / \mathfrak{m}_{1}^{i} \text { (efficient) : }\left.\right|_{1} ^{i} .
$$

A special situation exists for the case $i=1$. As already noted, the $\Lambda$-module $k$ corresponds to the second through fourth diagrams displayed in (3.4.1); and the third and fourth are both efficient diagrams for $k$. This holds because the two nonisomorphic $\Gamma$-modules $\Gamma_{1} / \mathfrak{m}_{1}$ and $\Gamma_{2} / \mathfrak{m}_{2}$ (both isomorphic to $k$ as rings) become isomorphic when considered as $\Lambda$-modules.

The way that homomorphisms of $\Lambda$-modules arise from homomorphisms of $\Gamma$-modules is given in our final result. (We do not know the extent to which this theorem holds if nonstandard diagrams are used.) 
Theorem 3.8. Let $\mathcal{D}^{\prime}$ and $\mathcal{D}$ be disjoint unions of block cycle and deleted cycle diagrams, and let $f: M\left(\mathcal{D}^{\prime}\right) \rightarrow M(\mathcal{D})$ be a $\Lambda$-homomorphism. Then there is a $\Gamma$-homomorphism $f^{* *}: X\left(\mathcal{D}^{\prime}\right) \rightarrow X(\mathcal{D})$ whose restriction $f^{*}$ to $S\left(\mathcal{D}^{\prime}\right)$ is a $\Lambda$-homomorphism: $S\left(\mathcal{D}^{\prime}\right) \rightarrow S(\mathcal{D})$ that takes $K\left(\mathcal{D}^{\prime}\right) \rightarrow K(\mathcal{D})$ and induces $f$. Moreover, if $f$ is one-to-one or onto, then so is any such $f^{*}$.

\section{Separated covers and almost functorial property.}

Notation 4.1. The rings considered in this section are much more general than in the rest of this paper. In particular, rings in this section are not necessarily commutative.

In the fixed notation in this section we assume that we have the following commutative diagram of rings:

$$
\begin{aligned}
& \Lambda \subseteq \Gamma \quad(\Gamma \Gamma \text { noetherian }) \\
& \downarrow_{\downarrow} \quad \downarrow^{\rho} \quad(\text { ker }=C) \\
& \bar{\Lambda} \subseteq \bar{\Gamma}(\bar{\Lambda} \text { semisimple artinian, } \bar{\Lambda} \bar{\Gamma} \text { finitely generated })
\end{aligned}
$$

where these rings satisfy the following conditions (i) $-(\mathrm{v})$.

(i) The ring $\Gamma$ is left noetherian;

(ii) the map $\rho: \Gamma \rightarrow \bar{\Gamma}$ and its restriction $\rho: \Lambda \rightarrow \bar{\Lambda}$ are both surjective ring homomorphisms with the same kernel $C$;

(iii) the ring $\bar{\Lambda}$ is semisimple artinian;

(iv) the $\bar{\Lambda}$-module $\bar{\Gamma}$ is finitely generated; and

(v) $\Lambda=\{x \in \Gamma \mid \rho(x) \in \bar{\Lambda}\}$, the pullback of this diagram.

Thus, diagram (4.1.1) is a conductor square defining $\Lambda$ as a subring of $\Gamma$, and $C$ is a conductor ideal for $\Lambda$ and $\Gamma$. Since the hypotheses on $\Lambda$ are not left-right symmetric, the term module means "left module" unless otherwise stated. We include some reminders of this near the beginning of this section.

Lemma 4.2. The ring $\Lambda$ is left noetherian.

Proof. First note that ${ }_{\Lambda} \Gamma$ is finitely generated since ${ }_{\Lambda}(\bar{\Gamma} / \bar{\Lambda})$ is finitely generated. Let $L$ be any left ideal of $\Lambda$. Since ${ }_{\Gamma} \Gamma$ is noetherian, ${ }_{\Gamma}(C L)$ is finitely generated. Since ${ }_{\Lambda} \Gamma$ is finitely generated, so therefore is ${ }_{\Lambda}(C L)$. Thus it suffices to show that $\Lambda(L / C L)$ is finitely generated. Since $C$ is a 2 -sided ideal of $\Lambda$, this last $\Lambda$-module is a module over the artinian ring $\bar{\Lambda}=\Lambda / C$; and it is a submodule of the finitely generated $\bar{\Lambda}$-module $(\Gamma L) /(C L)$, hence is itself finitely generated.

The purpose of this section is to show how all finitely generated left $\Lambda$ modules and their homomorphisms can be built from $\Gamma$-modules and homomorphisms.

Definition 4.3. We call a (left) $\Lambda$-module $S$ separated - $\Gamma$-separated if additional precision is required - if $S$ is a $\Lambda$-submodule of some left $\Gamma$-module, 
say $X$. In this situation $\Gamma S$ denotes the $\Gamma$-submodule of $X$ generated by $S$. Unless $X$ is specified, $\Gamma S$ is not well-defined, not even up to isomorphism [Example 6.2]. However, there is always a canonical choice for $\Gamma S$, namely $\Gamma \otimes_{\Lambda} S$. This follows from the following simple fact, which we record as a lemma for future use.

For examples of nonseparated modules, see Examples 4.16.

Lemma 4.4. The $\Lambda$-module $S$ is separated (if and) only if the natural map $S \rightarrow \Gamma \otimes_{\Lambda} S$ is one-to-one.

Proof. Let $X$ be any $\Gamma$-module containing $S$. Then we can consider the composite map $S \rightarrow \Gamma \otimes S \rightarrow X$, where the second arrow denotes the map $\omega \otimes s \rightarrow \omega s$. The nontrivial half of the lemma holds because the composite map is the identity on $S$.

When we write $\Gamma S=\Gamma \otimes S$, for a $\Lambda$-module $S$, we mean that the natural map from the right-hand side to the left-hand side is a bijection, and $\otimes=$ $\otimes_{\Lambda}$. One easily checks that the following simple properties hold.

Lemma 4.5. Let $S, S^{\prime}$ be separated $\Lambda$-modules, regarded as $\Lambda$-submodules of $\Gamma S=\Gamma \otimes S$ and $\Gamma S^{\prime}=\Gamma \otimes S^{\prime}$, respectively. Then:

(i) Every $\Lambda$-homomorphism $f: S \rightarrow S^{\prime}$ can be uniquely extended to a $\Gamma$ homomorphism $\Gamma S \rightarrow \Gamma S^{\prime}$ (namely to $1 \otimes f$ ).

(ii) If $B$ is any left ideal of $\Gamma$ contained in $\Lambda$, then $B S$ is a $\Gamma$-submodule of $S$.

Caution. Statement (ii) becomes false without the hypothesis that $S$ is separated. The point is that we have $\gamma(b s)=(\gamma b) s$ provided that both sides are defined, but unless $S$ is contained in some $\Gamma$-module, the left-hand side is undefined.

Definition 4.6. We define a separated cover of a (left) $\Lambda$-module $M$ to be a surjective $\Lambda$-module homomorphism $\phi: S \rightarrow M$ in which $S$ is separated and "as close as possible to $M$ " in the following sense. If $S \rightarrow S^{\prime} \rightarrow M$ is any factorization of $\phi$, and $S^{\prime}$ is a separated $\Lambda$-module, then the map $S \rightarrow S^{\prime}$ is one-to-one (and therefore an isomorphism). Thus, passing from $S$ to $S^{\prime}$ gets no closer to $M$.

The starting point of our theory is the following triviality, since $\Lambda$ is left noetherian.

Proposition 4.7. Every finitely generated (left) $\Lambda$-module has a separated cover.

Proof. Let $\phi^{\prime}: F \rightarrow M$ be a homomorphism of any finitely generated separated (e.g. free) $\Lambda$-module onto the given $\Lambda$-module $M$. 
Since the $\Lambda$-module $F$ is noetherian, it has a submodule $H$ that is maximal with respect to the property that $H \subseteq \operatorname{ker}\left(\phi^{\prime}\right)$ and $F / H$ is a separated $\Lambda$ module. Then the $\Lambda$-module homomorphism $\phi: S=F / H \rightarrow M$ is easily seen to be a separated cover of $M$.

Remarks 4.8. (i) If $\Lambda$ is strictly split Dedekind-like, then we can drop the "finitely generated" hypothesis in the previous lemma. In fact, this is true in the much more general (nonlocal) setting described in $[\mathbf{L}]$, and for the noncommutative integral group ring studied in $[\mathbf{K}]$. But we do not know whether it is true in the present setting, (in particular, for unsplit Dedekindlike rings).

(ii) The remaining results in this section do not have finite generation hypotheses; that is, when separated covers of infinitely generated modules exist, they behave as described in the rest of this section.

Lemma 4.9. Let $\phi: S \rightarrow M$ be a $\Lambda$-module surjection, with $S$ separated. Then the following two conditions are equivalent.

(i) $\phi$ is a separated cover of $M$.

(ii) (a) $\operatorname{ker}(\phi)$ has no nonzero $\Gamma$-submodules (and hence $C \operatorname{ker}(\phi)=0$, so that $\operatorname{ker}(\phi)$ is canonically a $\bar{\Lambda}$-module); and

(b) $\operatorname{ker}(\phi) \subseteq C S$.

Proof. Let $X=\Gamma \otimes S$, which contains $S$ by Lemma 4.4.

(ii) $\Rightarrow$ (i). Consider any factorization $\phi: S \stackrel{\theta}{\rightarrow} S^{\prime} \rightarrow M$ with $S^{\prime}$ separated, and suppose that $\operatorname{ker}(\theta)$ contains a nonzero element $s$. Then $s \in \operatorname{ker}(\phi) \subseteq$ $C S$, so there is an expression $s=\sum_{i} c_{i} s_{i}$ with each $c_{i} \in C$ and $s_{i} \in S$.

On the other hand, $\operatorname{ker}(\phi)$ contains no nonzero $\Gamma$-submodules. Therefore, for some $\omega \in \Gamma$, the element $\omega s$ of $X$ is not an element of $\operatorname{ker}(\phi)$. But $\omega s=\sum_{i}\left(\omega c_{i}\right) s_{i}$, an element of $S$ since $C$ is an ideal of $\Gamma$ contained in $\Lambda$. Therefore $\phi(\omega s)$ is defined, and is nonzero since $\omega s \notin \operatorname{ker}(\phi)$. It follows that $\theta(\omega s) \neq 0$. However, $\theta$ can be viewed as a $\Gamma$-homomorphism $\Gamma S \rightarrow \Gamma S^{\prime}$ by Lemma 4.5. This yields the contradiction $0 \neq \theta(\omega s)=\omega \theta(s)=0$, proving (i).

(i) $\Rightarrow$ (ii)(a). Let $H$ be any $\Gamma$-submodule of $\operatorname{ker}(\phi)$. Then there is a factorization $\phi: S \rightarrow S / H \rightarrow M . S / H$ is a separated $\Lambda$-module, since $X / H$ is a $\Gamma$-module containing $S / H$. Hence, by the definition of separated cover, the map $S \rightarrow S / H$ must be one-to-one; that is, $H=0$ as desired.

Since $C \operatorname{ker}(\phi)$ is a $\Gamma$-submodule of $\operatorname{ker}(\phi)$, the previous paragraph shows that $C \operatorname{ker}(\phi)=0$, as claimed in (ii)(a).

Note that we have not yet used the standing hypothesis that the ring $\bar{\Lambda}$ is semisimple. The consequence of the semisimplicity of $\bar{\Lambda}$ that we need below is that every $\bar{\Lambda}$-module is semisimple.

(i) $\Rightarrow$ (ii)(b). Suppose, by way of contradiction, that $\operatorname{ker}(\phi) \nsubseteq C S$. Since $C \operatorname{ker}(\phi)=0, \operatorname{ker}(\phi)$ is a $\bar{\Lambda}$-module and hence is a semisimple $\Lambda$-module. 
Therefore, it has a simple submodule $U$ that is not contained in $C S$. Let $\nu$ be the natural homomorphism of $S$ onto $S / C S$. Since the $\Lambda$-module $S / C S$ is annihilated by $C$, it is a $\bar{\Lambda}$-module and hence is semisimple. Therefore there is a projection map $\pi: S / C S \rightarrow \nu(U)$. Moreover, since $U$ is simple and not contained in $C S, \nu$ is one-to-one on $U$. Therefore $\nu^{-1}: \nu(U) \rightarrow U$ is a well-defined $\Lambda$-module map. The composition of the maps

$$
S \stackrel{\nu}{\longrightarrow} S / C S \stackrel{\pi}{\longrightarrow} \nu(U) \stackrel{\nu^{-1}}{\longrightarrow} U
$$

is a map $S \rightarrow U$ that equals the identity on $U$. Therefore $U$ is a direct summand of $S$, say $S=U \oplus T$. Then $S / U \cong T$ is again a separated $\Lambda$ module. Since $U \subseteq \operatorname{ker}(\phi)$ there is a factorization $\phi: S \rightarrow S / U \rightarrow M$. Since $\phi$ is a separated cover, we obtain the contradiction $U=0$, proving that statement (ii)(b) holds.

Lemma 4.10. Let $\phi: S \rightarrow M$ be a separated cover. Then $\phi$ is a minimal epimorphism (i.e., if $T$ is a $\Lambda$-submodule of $S$ such that $\phi(T)=M$, then $T=S)$.

Proof. Let $T$ be as above. Then $T+\operatorname{ker}(\phi)=S$. Since $\operatorname{ker}(\phi)$ is a $\bar{\Lambda}$-module [Lemma 4.9], it is semisimple. Therefore we have $\operatorname{ker}(\phi)=(T \cap \operatorname{ker}(\phi)) \oplus K$ for some submodule $K$, and therefore $T \oplus K=S$. This yields a factorization $\phi: S \rightarrow S / K \cong T \rightarrow M$ with $S / K \cong T$ separated, showing that $K=0$ and therefore $T=S$.

Proposition 4.11. If $\phi: S \rightarrow M$ is a separated cover and $M$ is finitely generated, then so is $S$.

Proof. Choose pre-images $s_{1}, \ldots, s_{n} \in S$ of some finite set of generators of $M$. Then $\phi\left(\sum_{i} \Lambda s_{i}\right)=M$. Since $\phi$ is a minimal epimorphism we have $\sum_{i} \Lambda s_{i}=S$, as desired.

Theorem 4.12 (Almost functorial property). Let $f: N \rightarrow M$ be a $\Lambda$-module homomorphism, and let $\phi^{\prime}, \phi$ be separated covers. Then $f$ can be lifted to a $\Lambda$-homomorphism $\theta$ such that the following diagram commutes.

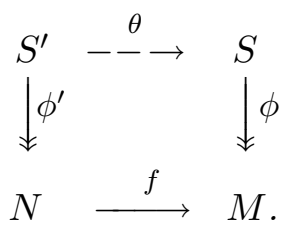

If $f$ is one-to-one or onto, then any such $\theta$ has the same property.

Proof. Since $S^{\prime} / C S^{\prime}$ is a module over the semisimple artinian ring $\bar{\Lambda}$, it has a decomposition $S^{\prime} / C S^{\prime}=\oplus_{i} \Lambda \bar{x}_{i}^{\prime}$ where each $\Lambda \bar{x}_{i}^{\prime} \cong \bar{\Lambda}_{i}$ for some idempotent element $\bar{e}_{i} \in \bar{\Lambda}$. We can choose each $\bar{x}_{i}^{\prime}$ such that

$$
\bar{e}_{i} \rightarrow \bar{x}_{i}^{\prime} \quad \text { under the isomorphism } \bar{\Lambda} \bar{e}_{i} \cong \bar{\Lambda} \bar{x}_{i}^{\prime} .
$$


Choose a pre-image $e_{i} \in \Lambda$ of each $\bar{e}_{i}$ and $x_{i}^{\prime} \in S^{\prime}$ of $\bar{x}_{i}^{\prime}$. Let $\left\{c_{j} s_{j}^{\prime}\right\}$ (with $\left.c_{j} \in C\right)$ be a set of generators of the $\Lambda$-module $C S^{\prime}$. Then choose elements $x_{i}, s_{j}$ related to the previously chosen elements as follows.

$$
f \phi^{\prime}\left(x_{i}^{\prime}\right)=\phi\left(x_{i}\right) \quad \text { and } \quad f \phi^{\prime}\left(s_{j}^{\prime}\right)=\phi\left(s_{j}\right) .
$$

Since $\bar{e}_{i}$ is idempotent we have $\bar{e}_{i} \bar{x}_{i}^{\prime}=\bar{x}_{i}^{\prime}$ and therefore the elements $e_{i} x_{i}^{\prime}$ together with $c_{j} s_{j}^{\prime}$ generate $S^{\prime}$. If we can define a $\Lambda$-homomorphism $\theta$ that sends each $e_{i} x_{i}^{\prime} \rightarrow e_{i} x_{i}$ and $c_{j} s_{j}^{\prime} \rightarrow c_{j} s_{j}$ then we have lifted $f$. Let

$$
y^{\prime}=\sum_{i} \lambda_{i} e_{i} x_{i}^{\prime}+\sum_{j} \delta_{j} c_{j} s_{j}^{\prime} \quad \text { and } \quad y=\sum_{i} \lambda_{i} e_{i} x_{i}+\sum_{j} \delta_{j} c_{j} s_{j}
$$

where the sums are finite and each $\lambda, \delta \in \Lambda$. To see that $\theta$ is well-defined it suffices to show that $y^{\prime}=0 \Rightarrow y=0$. Since $\operatorname{ker}(\phi)$ contains no nonzero $\Gamma$-submodules, by Lemma 4.9, it suffices to show that $\Gamma y \subseteq \operatorname{ker}(\phi)$. Thus we suppose that $y^{\prime}=0$ and choose $\omega \in \Gamma$. Our objective to show that $\phi(\omega y)$ is defined and equal to zero.

We must be careful about two things: Elements of $N$ and $M$ cannot be multiplied by $\omega$, if these $\Lambda$-modules are not contained in $\Gamma$-modules; and consequently the $\Lambda$-homomorphisms $\phi^{\prime}, \phi$ need not be extendable to T-homomorphisms.

Reading the statement $y^{\prime}=0$ modulo $C S^{\prime}$ and using the expression in (4.12.4) for $y^{\prime}$ yields $0=\sum_{i} \lambda_{i} \bar{e}_{i} \bar{x}_{i}^{\prime}$. Directness of the sum $\oplus_{i} \Lambda \bar{x}_{i}^{\prime}$ then yields $\lambda_{i} \bar{e}_{i} \bar{x}_{i}^{\prime}=0$ for all $i$. Hence, by the isomorphism in (4.12.2), we have $\bar{\lambda}_{i} \bar{e}_{i}=0$. Therefore each $\lambda_{i} e_{i} \in C$. Since $C$ is an ideal of $\Gamma$ contained in $\Lambda$, we have $\omega \lambda_{i} e_{i} x_{i}^{\prime} \in C S^{\prime} \subseteq S^{\prime}$, and therefore $\phi^{\prime}\left(\omega \lambda_{i} e_{i} x_{i}^{\prime}\right)$ is defined. Similarly $\phi^{\prime}\left(\omega \delta_{j} c_{j} s_{j}^{\prime}\right)$ is defined. Therefore $y^{\prime}=0$ yields

$$
0=f \phi^{\prime}\left(\omega y^{\prime}\right)=\sum_{i} f \phi^{\prime}\left(\omega \lambda_{i} e_{i} x_{i}^{\prime}\right)+\sum_{j} f \phi^{\prime}\left(\delta_{j} c_{j} s_{j}^{\prime}\right) .
$$

Since each $\omega \lambda_{i} e_{i} \in C \subseteq \Lambda$, relations (4.12.3) show that

$$
f \phi^{\prime}\left(\omega \lambda_{i} e_{i} x_{i}^{\prime}\right)=\omega \lambda_{i} e_{i} f \phi^{\prime}\left(x_{i}^{\prime}\right)=\omega \lambda_{i} e_{i} \phi\left(x_{i}\right)=\phi\left(\omega \lambda_{i} e_{i} x_{i}\right) .
$$

Similarly we have $f \phi^{\prime}\left(\omega \delta_{j} c_{j} s_{j}^{\prime}\right)=\phi\left(\omega \delta_{j} c_{j} s_{j}\right)$. Making these replacements in (4.12.5) now shows that $\phi(\omega y)=0$, completing the proof that $\theta$ is welldefined.

Suppose that $f$ is onto. Then commutativity of diagram (4.12.1) shows that $\phi \theta\left(S^{\prime}\right)=M$. Since $\phi$ is a minimal epimorphism [Lemma 4.10] we have $\theta\left(S^{\prime}\right)=S$, as claimed in the theorem.

On the other hand, suppose that $f$ is one-to-one. Then $f \phi^{\prime}$ is a separated cover of $f(N)$ by $S^{\prime}$. Therefore the factorization $f \phi^{\prime}=\phi \theta: S^{\prime} \rightarrow \theta\left(S^{\prime}\right) \rightarrow$ $f(N)$ shows (by the definition of "separated cover") that $\theta$ is one-to-one. 
Corollary 4.13 (Uniquenes of separated cover). Let $\phi^{\prime}: S^{\prime} \rightarrow M$ and $\phi: S$ $\rightarrow M$ be separated covers of a $\Lambda$-module $M$. Then there is an isomorphism $\theta: S^{\prime} \cong S$ such that $\phi^{\prime}=\phi \theta$.

Proof. Take $N=M$ and $f=1$ in (4.12.1). Then apply the almost functorial property.

Corollary 4.14. Let $\phi: S \rightarrow M$ be a separated cover and $f: S^{\prime} \rightarrow M$ any surjective $\Lambda$-homomorphism such that $S^{\prime}$ is a separated $\Lambda$-module. Then $f$ factors through $\phi$; that is, there is a surjective $\Lambda$-homomorphism $\theta$ such that $f$ factors as follows:

$$
f: \quad S^{\prime} \stackrel{\theta}{\rightarrow} S \stackrel{\phi}{\rightarrow} M .
$$

Proof. The Corollary follows from Theorem 4.12 if we take $N=S^{\prime}$ and note that the identity map $\phi^{\prime}$ on $S^{\prime}$ is a separated cover.

Corollary 4.15. Let $\phi: S \rightarrow M$ be a separated cover such that $\operatorname{ker}(\phi) \neq 0$. Then $M$ is not a separated module.

Proof. If $M$ were a separated module, then the identity map on $M$ would be a projective cover of $M$. Therefore, by uniqueness of separated covers (Corollary 4.13), we would have $\operatorname{ker}(\phi)=0$.

Examples 4.16 (nonseparated modules). (i) For the two simplest examples, let $\Lambda$ be an unsplit Dedekind-like ring with normalization $\Gamma$. Then $\mathfrak{m} / \mathfrak{m}^{2}$ is $\Gamma$-isomorphic to $\Gamma / \mathfrak{m}=F$, and therefore this simple $\Gamma$-module has length 2 as a $\Lambda$-module. Therefore there is a $\Lambda$-module $K$ strictly contained in $\mathfrak{m}$ and strictly containing $\mathfrak{m}^{2}$.

The natural maps $\Gamma / \mathfrak{m}^{2} \rightarrow \Gamma / K$ and $\Lambda / \mathfrak{m}^{2} \rightarrow \Lambda / K$ are separated covers [Lemma 4.9 with $C=\mathfrak{m}$ ] and have nonzero kernels. Therefore the $\Lambda$-modules $\Gamma / K$ and $\Lambda / K$ are not separated modules [Corollary 4.15].

(ii) Much more generally, let $M=M(\mathcal{D})$ be the $\Lambda$-module associated with any standard diagram. (See (2.4.1), (2.6.2), and (3.3.1).) We claim that, if any bottom-gluing or bottom-reduction actually occurs, then $M$ is not a separated module.

For the proof, let $S=S(\mathcal{D})$, in the notation of Subsections 2.2 and 3.2. Then the natural map $\phi: S(\mathcal{D}) \rightarrow M(\mathcal{D})$ is a separated cover. (See Subsections 9.6 and 10.4.) The statement that bottom-gluing or bottom-reduction actually occurs is equivalent to the statement that $\operatorname{ker}(\phi) \neq 0$. Therefore, by Corollary 4.15, $M$ is not a separated module.

Remark 4.17. Before proceeding to the next section, we comment on the reason for the word "separated." Let $\phi: S \rightarrow M$ be a separated cover. If $\Lambda$ is strictly split Dedekind-like, $\operatorname{ker}(\phi)$ is always an amalgamation relation. (See diagrams (3.3.1), in which the passage from $S$ to $M$ is always given by what we call "bottom-gluing" relations.) Thus a $\Lambda$-module is "separated" if no 
such amalgamation relation has been imposed. In the unsplit case, $\operatorname{ker}(\phi)$ is often, but not always an amalgamation. (See diagrams (2.4.1) and (2.6.2), in which the passage from $S$ to $M$ involves both bottom gluing and bottom reduction.) Thus our terminology is slightly misleading in the context of unsplit Dedekind-like rings.

\section{Isomorphism as matrix problem, unsplit case.}

The main results of this section use separated covers to transform the problem of describing isomorphism classes of $\Lambda$-modules into a matrix problem over the fields $k$ and $F$, in the unsplit case.

Notation 5.1. Throughout this section $\Lambda$ is a pullback ring, as specified in diagram (1.1.1) and display (1.1.2). That is, the first few results apply to both the unsplit and strictly split case, but, beginning with Notation 5.6, we assume that $(\Lambda, \mathfrak{m}, k)$ is unsplit Dedekind-like with normalization $(\Gamma, \mathfrak{m}, F)$.

Recall: (i) For a $\Lambda$-submodule $S$ of a $\Gamma$-module $X$ we write $\Gamma S=\Gamma \otimes_{\Lambda} S$ to mean that the natural surjection of the right-hand side onto the left-hand side is a bijection. (ii) A separated $\Lambda$-module is any $\Lambda$ submodule of some $\Gamma$-module. (iii) We write functions as right operators when they represent (or will represent) right multiplication by matrices.

Lemma 5.2. Let $S \neq 0$ be a separated, finitely generated $\Lambda$-module and $\Gamma S$ a $\Gamma$-module generated by $S$. Then $\Gamma S=\Gamma \otimes_{\Lambda} S$ if and only if there is a positive integer $n$ such that $S / \mathfrak{m} S \cong k^{(n)}$ (free $k$-module of rank $n$ ) and $\Gamma S / \mathfrak{m} S \cong \bar{\Gamma}^{(n)}$ (free $\bar{\Gamma}$-module of rank $\left.n\right)$.

Proof. Let $\tau: \Gamma \otimes_{\Lambda} S \rightarrow \Gamma S$ be the natural surjection. Note that, since $\mathfrak{m}$ is an ideal of both rings $\Lambda$ and $\Gamma$, we have $\mathfrak{m}(\Gamma S)=\mathfrak{m} S$.

Suppose first that $S / \mathfrak{m} S \cong k^{(n)}$ and $\Gamma S / \mathfrak{m} S \cong \bar{\Gamma}^{(n)}$. The surjection $\tau$ induces a surjection $\bar{\tau}:(\Gamma \otimes S) / \mathfrak{m}(\Gamma \otimes S) \rightarrow \Gamma S / \mathfrak{m} S$. We claim that $\bar{\tau}$ is a bijection. Tensoring the short exact sequence $\mathfrak{m} \hookrightarrow \Gamma \rightarrow \bar{\Gamma}$ by $S$ and using right-exactness of the tensor product yields the following chain of isomorphisms.

$$
\frac{\Gamma \otimes S}{\mathfrak{m}(\Gamma \otimes S)} \cong \frac{\Gamma}{\mathfrak{m}} \otimes_{\Lambda} S \cong \frac{\Gamma}{\mathfrak{m}} \otimes_{\Lambda} \frac{S}{\mathfrak{m} S} \cong \bar{\Gamma} \otimes_{k} k^{(n)} \cong \bar{\Gamma}^{(n)}
$$

Since $\bar{\tau}$ is a $\Gamma$-module surjection from the left-hand side of (5.2.1) onto $\Gamma S / \mathfrak{m} S \cong \bar{\Gamma}^{(n)}$, and $\bar{\Gamma}$ is a $\bar{\Gamma}$-module of finite length, we see that $\bar{\tau}$ is a bijection, as claimed.

Now we lift this assertion to $\tau$ itself. Take $x \in \operatorname{ker}(\tau)$. Let $\bar{x}$ be the image of $x$ in the left-hand side of (5.2.1). Since $\bar{\tau}$ is an isomorphism, we have $\bar{x}=0$, that is, $x \in \mathfrak{m}(\Gamma \otimes S)$. Therefore there is an expression $x=\sum_{i} m_{i} \otimes s_{i}\left(m_{i} \in \mathfrak{m}, s_{i} \in S\right)$. Since $\mathfrak{m} \subseteq \Lambda$ we have $x=1 \otimes\left(\sum_{i} m_{i} s_{i}\right)$. 
But $x \in \operatorname{ker}(\tau)$ implies that $\sum_{i} m_{i} s_{i}=0$. Therefore $x=0$; and so $\tau$ is an injection, hence a bijection.

Conversely, suppose that $\Gamma S=\Gamma \otimes S$. Then by right exactness of the tensor product, as in the first part of this proof, we have $\Gamma S / \mathfrak{m} S \cong \bar{\Gamma} \otimes$ $(S / \mathfrak{m} S)$. Since $S / \mathfrak{m} S$ is a module over the field $\Lambda / \mathfrak{m}=k$ we have $S / \mathfrak{m} S \cong$ $k^{(n)}$ for some $n$, and hence $\bar{\Gamma} \otimes_{\Lambda}(S / \mathfrak{m} S) \cong \bar{\Gamma} \otimes_{k} k^{(n)} \cong \bar{\Gamma}^{(n)}$ as desired.

Example 5.3. Caution. We have $\Gamma \cdot \Gamma=\Gamma$. However, when $\Lambda$ is unsplit or strictly split Dedekind-like, we do not have $\Gamma \cdot \Gamma=\Gamma \otimes_{\Lambda} \Gamma$. If this equality held, the previous lemma, with $S=\Gamma$, would require $\Gamma / \mathfrak{m} \Gamma$ to be $\Lambda$-isomorphic to $k^{(n)}$ for some $n$ and $\Gamma$-isomorphic to $\bar{\Gamma}^{(n)}$ for this same $n$. This would imply that $k=\bar{\Gamma}$, which never holds when $\Lambda \neq \Gamma$.

This fact will cause some inconvenience in the remainder of this paper.

Corollary 5.4. Let $X$ be a finitely generated $\Gamma$-module. Then $X$ has a $\Lambda$ submodule $S$ such that $X=\Gamma \otimes_{\Lambda} S$ if and only if $X / \mathfrak{m} X$ is a free $\bar{\Gamma}$-module.

Remark. Note that if $\Lambda$ is unsplit Dedekind-like, the phrase "if and only if $X / \mathfrak{m} X$ is a free $\bar{\Gamma}$-module" can be deleted from the statement of Corollary 5.4, because all modules over the field $\bar{\Gamma}=F$ are free.

Proof. The "only if" assertion is an immediate consequence of Lemma 5.2.

Conversely, suppose that $X / \mathfrak{m} X \cong \bar{\Gamma}^{(n)}$ for some integer $n$. Choose $n$ elements $x_{1}, \ldots, x_{n} \in X$ whose images in $\bar{\Gamma}^{(n)}$ are a free $\bar{\Gamma}$-basis of $\bar{\Gamma}^{(n)}$, and let $S=\sum_{i} \Lambda x_{i}$. Since $\mathfrak{m} \subseteq \operatorname{rad} \Gamma$, Nakayama's lemma shows that $\Gamma S=X$.

Since $S / \mathfrak{m} S$ is a $k=\Lambda / \mathfrak{m}$-vector space generated by $n$ elements, we have $S / \mathfrak{m} S \cong k^{(m)}$ for some $m \leq n$. It now suffices, by Lemma 5.2 , to show that $m=n$. So suppose that $m<n$. Then the $\bar{\Gamma}$-module $\Gamma(S / \mathfrak{m} S)=\Gamma S / \mathfrak{m} S \cong$ $\bar{\Gamma}^{(n)}$ would be generated by $m<n$ elements, which is impossible for a free module of rank $n$ over an artinian ring.

Lemma 5.5. Let $Y$ be a $k$-subspace of some finitely generated $\bar{\Gamma}$-module, and suppose $\Lambda \neq \Gamma$.

(i) If $\bar{\Gamma} Y=\bar{\Gamma} \otimes_{k} Y$, then $Y$ contains no nonzero $\bar{\Gamma}$-submodule.

(ii) If $Y$ contains no nonzero $\bar{\Gamma}$-module and $\operatorname{dim}_{k}(\bar{\Gamma})=2$ (which holds whenever $\Lambda$ is Dedekind-like), then $\bar{\Gamma} Y=\bar{\Gamma} \otimes_{k} Y$.

Proof. (i) Let $d=\operatorname{dim}_{k}(\bar{\Gamma})$. Since $\Lambda \neq \Gamma$ we have $d>1$. Let $G$ be the largest $\bar{\Gamma}$-submodule of $Y$. Then $Y=G \oplus V$ for some $k$-subspace $V$ of $Y$. Let $y, g, v$ be the $k$-dimensions of $Y, G, V$ respectively, so that $y=g+v$.

We have $\operatorname{dim}_{k}(\bar{\Gamma} Y) \leq \operatorname{dim}_{k}(G)+\operatorname{dim}_{k}(\bar{\Gamma} V) \leq g+d v$. Also, $\operatorname{dim}_{k}(\bar{\Gamma} \otimes Y)=$ $d y=d g+d v$. Since $\operatorname{dim}_{k}(\bar{\Gamma} Y)=\operatorname{dim}_{k}(\bar{\Gamma} \otimes Y)$ by (i), we have $d g \leq g$. But then $d>1$ implies that $g=0$ as desired.

(ii) Let $\varepsilon \in \bar{\Gamma}-k$. Since $\operatorname{dim}_{k} \bar{\Gamma}=2$ we have $\bar{\Gamma}=k+k \varepsilon$. Hence 1 and $\varepsilon$ are $k$-linearly independent and $\varepsilon^{2} \in k+k \varepsilon$. We want to show that the 
natural surjection $\tau: \bar{\Gamma} \otimes_{k} Y \rightarrow \bar{\Gamma} Y$ is a monomorphism. Let $x=\sum_{i} \gamma_{i} \otimes y_{i} \in$ $\operatorname{ker}(\tau)$. We may assume that the $y_{i}$ are linearly independent over $k$. Write $\gamma_{i}=\alpha_{i}+\beta_{i} \varepsilon\left(\alpha_{i}, \beta_{i} \in k\right)$.

Then $0=\tau(x)=\left(\sum_{i} \alpha_{i} y_{i}\right)+\varepsilon\left(\sum_{i} \beta_{i} y_{i}\right)$ which we can write in the form $0=u+\varepsilon v$ with $u, v \in Y$. But then $k u+k v=k \varepsilon v+k v$ is a $\bar{\Gamma}$-submodule of $Y$. By assumption this $\bar{\Gamma}$-submodule must be 0 , and hence $u=v=0$. Linear independence of the $y_{i}$ over $k$ therefore implies that every $\alpha_{i}$ and $\beta_{i}$ equals zero, and therefore $x=0$, as desired.

Notation 5.6 (Matrix setup, unsplit case). For the remainder of this section, let $(\Lambda, \mathfrak{m}, k)$ be an unsplit Dedekind-like ring with normalization $(\Gamma, \mathfrak{m}$, $F)$, as in Notation 1.1. Thus $\bar{\Gamma}=F$, a 2-dimensional field extension of $k$.

The map $\rho: \Gamma \rightarrow F=\bar{\Gamma}$ in pullback diagram (1.1.1) induces a $\Gamma$-linear map $\Gamma / \mathfrak{m}^{t} \rightarrow F$ for every $t$, which we again call $\rho$. In fact, we denote any direct sum of such maps - from the direct sum of any $n$ such modules to $F^{(n)}$ — by $\rho$. Moreover, we write $\mathfrak{m}^{\infty}=0$, and therefore $\Gamma=\Gamma / \mathfrak{m}^{\infty}$.

When $t \neq \infty$, we have $F \cong \mathfrak{m}^{t-1} / \mathfrak{m}^{t}$ via the $\Gamma$-isomorphism $\gamma+\mathfrak{m} \rightarrow$ $\gamma \pi^{t-1}+\mathfrak{m}^{t}$, where $\pi$ is the standard $\Gamma$-generator of $\mathfrak{m}$ mentioned in Notation 1.1. Thus - given this choice of $\pi$ - every element of $F$ has a standard image in $\Gamma / \mathfrak{m}^{t}$, which we often regard as an identification, and the set of all such standard images of elements of $F$ defines the standard copy of $F$ in $\Gamma / \mathfrak{m}^{t}$. We note:

(5.6.1) When $t \neq \infty, \neq 1$, the standard copy of $F$ in $\Gamma / \mathfrak{m}^{t}$ satisfies $\rho(F)=0$.

Let $X$ be the nonzero (external) direct sum of $\Gamma$-modules displayed below.

$$
X=\oplus_{\nu=1}^{n} \Gamma / \mathfrak{m}^{t_{\nu}}
$$

where every $t_{\nu}$ is a positive integer or $\infty$. Since $\Gamma$ is a DVR, every finitely generated indecomposable $\Gamma$-module is isomorphic to such a module $X$.

Let $e_{\infty}$ be the number of summands $\Gamma / \mathfrak{m}^{t_{\nu}}$ of $X$ in (5.6.2) of infinite length $t_{\nu}$. The map $\rho$ yields the $\Gamma$-linear surjection to $F^{(n)}$ shown in (5.6.3).

$$
\rho: X \rightarrow F^{(n)} \quad F^{\left(n-e_{\infty}\right)} \subseteq X .
$$

We call coordinate $\nu$ of $F^{(n)}$ the coordinate corresponding to the the $\nu^{\text {th }}$ summand $\Gamma / \mathfrak{m}^{t_{\nu}}$ of $X$. Each of the $n-e_{\infty}$ summands $\Gamma / \mathfrak{m}^{t_{\nu}}$ of finite length contains a standard copy of $F$. This yields the inclusion shown in (5.6.3). Thus every coordinate of $F^{\left(n-e_{\infty}\right)}$ has a corresponding summand $\Gamma / \mathfrak{m}^{t_{\nu}}$ of $X$, namely the summand containing this copy of $F$.

A matrix setup $\mathcal{X}$ (for $\Lambda$ unsplit Dedekind-like) is any direct sum decomposition of the form $X$ in (5.6.2), together with the corresponding surjection and inclusion displayed in (5.6.3). The $\Gamma$-module associated with $\mathcal{X}$ is the module $X$ in (5.6.2). 
Notation 5.7 (Matrix pair $(A, B)$ ). Let $\mathcal{X}$ be a matrix setup (unsplit case). Let $A$ be an $n \times n$ matrix over $F=\bar{\Gamma}$, and let $B$ be a $q \times\left(n-e_{\infty}\right)$ matrix over $F$, for some $q \geq 0$. We attach a length label to each column of $A$ and $B$ as follows. For $1 \leq \nu \leq n$ define the length label of column $\nu$ of $A$ to be the length $t_{\nu}$ of the corresponding summand $\Gamma / \mathfrak{m}^{t_{\nu}}$ of $X$. For $1 \leq j \leq n-e_{\infty}$ define the length label of column $j$ of $B$ to be the length of the summand of $X$ corresponding to coordinate $j$ of $F^{\left(n-e_{\infty}\right)}$. Thus every column of $B$ has a corresponding column in $A$, namely the column of $A$ with the same corresponding summand of $X$; and every column of $A$ whose length label is finite has a corresponding column in $B$.

We require $A$ and $B$ to have the following properties.

(5.7.1) $\quad A$ is invertible; the rows of $B$ are linearly independent over $F$; and every column of $B$ whose length label is 1 consists of zeros.

Thus right multiplication by $A$ and $B$ define $k$-linear monomorphisms

$$
A: k^{(n)} \hookrightarrow F^{(n)} \quad B: k^{(q)} \hookrightarrow F^{\left(n-e_{\infty}\right)} .
$$

We call $(A, B)$ a matrix pair associated with $\mathcal{X}$.

Although we write the matrices of the ordered pair $(A, B)$ side by side, we think of $B$ as being written underneath $A$, with each column of $B$ written under its corresponding column in $A$, and the length label of each column of $A$ above that column. Thus columns of $A$ with length label $\infty$ have no corresponding column in $B$ written beneath them.

Definition 5.8 (Associated $\Lambda$-module). Suppose that $(A, B)$ is a matrix pair associated with the matrix setup $\mathcal{X}$. Using the maps in (5.7.2), let $S(A)$ be the $\Lambda$-module

$$
S(A)=\left\{x \in X \mid \rho(x) \in \operatorname{im}(A)=k^{(n)} \cdot A\right\}
$$

and define the $\Lambda$-module $M(A, B)$ by

$$
M(A, B)=S(A) / \operatorname{im}(B) \quad \text { where } \quad \operatorname{im}(B)=k^{(q)} \cdot B .
$$

(If $q=0$ we interpret this to mean that $M(A, B)=S(A)$.) This definition makes sense - that is, $\operatorname{im}(B) \subseteq S(A)$ - because $\rho(\operatorname{im}(B))=0$ by (5.6.1) and the requirement about columns of zeros in (5.7.1). We call $M(A, B)$ the $\Lambda$-module associated with the matrix pair $(A, B)$ (with respect to the matrix $\operatorname{setup} \mathcal{X})$.

We note that $\Gamma \cdot S(A)=X$, since $A$ is invertible. Thus $S(A)$ is the pullback of the commutative square in diagram (5.8.3) below.

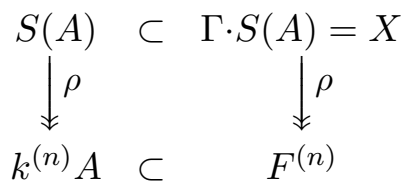


Note that $\operatorname{ker}(\rho)=\mathfrak{m} X=\mathfrak{m} S(A)$. (The second equality holds because $\mathfrak{m} \Gamma=\mathfrak{m}$.

Definition 5.9 (Display operation). A display operation on a matrix pair $(A, B)$ (associated with a matrix setup $\mathcal{X}$ ) is a permutation of the columns of $A$, together with the same permutation of their length labels and their corresponding columns of $B$ (so that corresponding columns of $A$ and $B$ remain corresponding columns after being moved). Thus each display operation corresponds to the effect on $(A, B)$ of rearranging the summands $\Gamma / \mathrm{m}^{t}$ in decomposition (5.6.2) of $X$. For future reference we record the following consequence of this observation.

(5.9.1) Let $\left(A^{\prime}, B^{\prime}\right)$ - including its length labels - be obtained by performing a display operation on $(A, B)$. Then $M\left(A^{\prime}, B^{\prime}\right) \cong M(A, B)$, where the module on the left is computed with respect to the correspondingly altered matrix setup.

We will need display operations to display our matrix pairs in canonical form.

Theorem 5.10. Let $\mathcal{X}$ be a matrix setup (unsplit case) with associated $\Gamma$ module $X$, and let $(A, B)$ be a matrix pair associated with $\mathcal{X}$. Also, let $S=S(A)$. Then:

(i) $\Gamma S=\Gamma \otimes_{\Lambda} S$.

(ii) The natural surjection $S \rightarrow M(A, B)$ is a separated cover of $M(A, B)$. Moreover,

(iii) Every finitely generated $\Lambda$-module is isomorphic to $M(A, B)$ for some $(A, B)$ with respect to some matrix setup $\mathcal{X}$.

(iv) Let $X$ and $X^{\prime}$ be the $\Gamma$-modules associated with matrix setups $\mathcal{X}$ and $\mathcal{X}^{\prime}$ respectively, and let $M(A, B)$ and $M\left(A^{\prime}, B^{\prime}\right)$ be $\Lambda$-modules computed with respect to $\mathcal{X}$ and $\mathcal{X}^{\prime}$ respectively. If $M(A, B) \cong M\left(A^{\prime}, B^{\prime}\right)$ as $\Lambda$-modules then $X \cong X^{\prime}$ as $\Gamma$-modules.

Proof. (i) This follows from Lemma 5.2 and (5.8.3).

(ii) By Lemma 4.9 (with $C=\mathfrak{m}$ ) it suffices to show: (a) $\operatorname{im}(B)$ contains no nonzero $\bar{\Gamma}$-submodules, and (b) $\operatorname{im}(B) \subseteq \mathfrak{m} S$. Consider $\bar{\Gamma} \cdot \operatorname{im}(B)$, the $\bar{\Gamma}$-module generated by the rows of $B$. We claim that $\bar{\Gamma} \cdot \operatorname{im}(B)=\bar{\Gamma} \otimes_{k} \operatorname{im}(B)$.

Since the right-hand side maps onto the left-hand side, it suffices to show that both sides have the same $F$-dimension. Since the rows of $B$ are $F$ linearly independent, by (5.7.1), the $F$-dimension of the left-hand side is the number of rows of $B$. On the other hand, $F$-independence of the rows of $B$ implies that they form a $k$ basis of $\operatorname{im}(B)=k^{(q)} B$, and hence an $F$-basis of $F \otimes_{k} \operatorname{im}(B)$; and hence the $F$-dimension of $F \otimes_{k} \operatorname{im}(B)$ again equals the number of rows of $B$, proving the claim. 
The claim, together with Lemma 5.5(i), shows that $\operatorname{im}(B)$ contains no nonzero $\bar{\Gamma}$-submodules, establishing (a).

To establish (b) it suffices to show that $\operatorname{im}(B)$ is contained in the sum of all those summands $\Gamma / \mathfrak{m}^{t}$ of $X$ whose length is finite but greater than 1 . The "finite" part is part of the definition of a matrix setup; and the "greater than 1" part follows from the fact that every column of $B$ with length label 1 consists of zeros, by (5.7.1).

(iii) Let $M$ be any finitely generated $\Lambda$-module. Then $M$ has a separated cover $\phi: S \rightarrow M$ [Proposition 4.7]. Since $S$ is a separated $\Gamma$-module, it can be regarded as a $\Lambda$-submodule of the $\Gamma$-module $X=\Gamma S=\Gamma \otimes_{\Lambda} S$ [Lemma 4.4]. Since $\Gamma$ is a DVR and ${ }_{\Gamma} X$ is finitely generated, we can take $X$ to be as shown in (5.6.2). This gives us modules $S$ and $\Gamma S=X$ to use in the top row of the commutative square in (5.8.3) that we are building. We will soon attach a matrix $A$ to $S$.

Decomposition (5.6.2) of $X$, together with the map $\rho$ in (5.6.3) yield an $F$-linear identification $\Gamma S / \mathfrak{m} S=F^{(n)}$. Since $\Gamma S=\Gamma \otimes_{\Lambda} S$ we have a $\Lambda$ isomorphism (equivalently, $k$-isomorphism) $S / \mathfrak{m} S \cong k^{(n)}$ [Lemma 5.2]. In addition, the relation $\Gamma S=\Gamma \otimes_{\Lambda} S$, reduced modulo $\mathfrak{m} S$ yields $F(S / \mathfrak{m} S)=$ $F \otimes_{k}(S / \mathfrak{m} S)$. Therefore the $n$-dimensional $k$-submodule $S / \mathfrak{m} S$ of $F^{(n)}=$ $\Gamma S / \mathfrak{m} S$ is $k$-generated by an $F$-basis of $F^{(n)}$. This yields an invertible $n \times n$ matrix $A$ over $F$ such that $S / \mathfrak{m} S=k^{(n)} A$.

To complete the proof that $S=S(A)$ it suffices to prove that $S$ is the pullback of this diagram, and for this it suffices to show that $S \supseteq \operatorname{ker}(\rho)=$ $\mathfrak{m} X$. But $\mathfrak{m} X=\mathfrak{m} \Gamma S=\mathfrak{m} S$, which is contained in $S$ since $\mathfrak{m} \subseteq \Lambda$.

It now suffices to prove that $\operatorname{ker}(\phi)=\operatorname{im}(B)$ for a suitable $B$. Since $\phi$ is a separated cover, Lemma 4.9 shows that $\operatorname{ker}(\phi)$ is a $k$-submodule of $X$; that is, $\mathfrak{m} \cdot \operatorname{ker}(\phi)=0$. Thus, in the current notation,

$$
\operatorname{ker}(\phi) \subseteq \oplus\left\{\mathfrak{m}^{t_{\nu}-1} / \mathfrak{m}^{t_{\nu}} \mid t_{\nu} \neq \infty\right\}=F^{\left(n-e_{\infty}\right)} .
$$

We claim that, for some nonnegative integer $q$, $\operatorname{ker}(\phi)$ has a $k$-basis consisting of $q$ elements of $F^{\left(n-e_{\infty}\right)}$ that are linearly independent over $F$. For this, it suffices (by a dimension argument) to show that $F \cdot \operatorname{ker}(\phi)=F \otimes_{k} \operatorname{ker}(\phi)$. But $\phi$ is a separated cover, so Lemma 4.9 shows that $\operatorname{ker}(\phi)$ has no nonzero $F$-submodules. Since $\Lambda$ is unsplit Dedekind-like, we have $\operatorname{dim}_{k}(F)=2$, so the desired equality follows by Lemma 5.5(ii).

In view of the claim, there is a $q \times\left(n-e_{\infty}\right)$ matrix $B$ over $F$ whose rows are $F$-linearly independent and form a $k$-basis of $\operatorname{ker}(\phi)$. One last appeal to Lemma 4.9 shows that $\operatorname{ker}(\phi) \subseteq \mathfrak{m} S=\mathfrak{m} \Gamma S=\mathfrak{m} X$. This shows that (5.10.1) can be refined to

$$
\operatorname{ker}(\phi) \subseteq \oplus\left\{\mathfrak{m}^{t_{\nu}-1} / \mathfrak{m}^{t_{\nu}} \mid t_{\nu} \neq \infty, \neq 1\right\} \subseteq F^{\left(n-e_{\infty}\right)}
$$

from which it follows that all columns of $B$ with length label 1 must be zero. Thus we now have $\operatorname{ker}(\phi)=k^{(n)} B$ were $B$ is as in (5.7.1), as desired. 
(iv) By uniqueness of the separated cover [Corollary 4.13], the $\Lambda$-isomorphism class of $S(A)$ is determined by that of $M(A, B)$. Statement (iv) now follows from the fact that, in any matrix setup, $X=\Gamma \otimes_{\Lambda} S(A)$, by statement (i) above.

The final step in transforming the problem of classifying isomorphism classes of finitely generated $\Lambda$-modules into a matrix problem is to transform the condition $M(A, B) \cong M\left(A^{\prime}, B^{\prime}\right)$ into a purely matrix-theoretic statement about the two matrix pairs $(A, B)$ and $\left(A^{\prime}, B^{\prime}\right)$. In view of statements (iii) and (iv) of the previous theorem, we can restrict our attention to the situation that both matrix pairs arise from the same matrix setup.

The notation $A$ [cols $j$ ] denotes the submatrix of $A$ consisting of the $j$ labeled columns.

Theorem 5.11 (Matrix operations, unsplit case). Let $(A, B)$ and $\left(A^{\prime}, B^{\prime}\right)$ be matrix pairs associated with a matrix setup $\mathcal{X}$ (where $\Lambda$ is unsplit Dedekind-like $)$. Then $M(A, B) \cong M\left(A^{\prime}, B^{\prime}\right)$ if and only if $\left(A^{\prime}, B^{\prime}\right)$ can be obtained from $(A, B)$ by a finite sequence of the following operations.

(i) (a) Left multiply $A$ by an invertible matrix over $k$.

(b) Left multiply $B$ by an invertible matrix over $k$.

(ii) For any length label $j$ right-multiply $A[$ cols $j]$ by an invertible matrix $Q$ over $F$ and, if $j \neq \infty$, simultaneously right-multiply $B$ [cols $j]$ by $Q$. (iii) (a) For any $i>j$ add an $F$-scalar multiple of a i-labeled column of $A$ to a $j$-labeled column of $A$. ( "Sweep toward smaller lengths in A.")

(b) For any $i<j$ add any $F$-scalar multiple of a $i$-labeled column of $B$ to a $j$-labeled column of $B$. ( "Sweep toward larger lengths in B.") We call these matrix operations " $k-F$ sweeping-similarity" operations.

Proof. The matrix operations in the theorem are stated in a way that is convenient for the use of the theorem and our eventual statement of the canonical form of $(A, B)$, but not for the proof of the present theorem. Therefore we begin the proof by changing notation.

Note that it is possible to perform a display operation, rearranging the summands $\Gamma / \mathfrak{m}^{t}$ of $X$ in descending order; that is, such that the decomposition (5.6.2) of $X$ takes the form

$$
\begin{gathered}
X=\left(\Gamma / \mathfrak{m}^{\infty}\right)^{\left(e_{\infty}\right)} \oplus\left(\Gamma / \mathfrak{m}^{t}\right)^{\left(e_{t}\right)} \oplus \ldots \oplus\left(\Gamma / \mathfrak{m}^{i}\right)^{\left(e_{i}\right)} \oplus \ldots \oplus(\Gamma / \mathfrak{m})^{\left(e_{1}\right)} \\
\text { where } \infty>t>\cdots>i>\cdots>1
\end{gathered}
$$

where any particular block, e.g. the $\infty$ block, might not actually be present. (Recall that the notation $\left(e_{t}\right)$ denotes the multiplicity of $\Gamma / \mathfrak{m}^{t}$ in $X$.)

After doing this display operation we can restate the stated sweepingsimilarity operations as relations of the form

$$
A^{\prime}=P_{1} A Q_{1} \quad B^{\prime}=P_{2} B Q_{2}
$$


in which $P_{1}$ and $P_{2}$ are invertible matrices over the field $k$, and $Q_{1}$ and $Q_{2}$ are block triangular invertible matrices over the field $F$. The block triangular forms of the matrices $Q_{1}$ and $Q_{2}$ are due to the fact that all sweeping in $A$ is toward smaller lengths while all sweeping in $B$ is toward greater lengths. We describe this block triangular form precisely in the next subsection, and then state and prove the theorem in the altered notation.

Notation 5.12 ( $\mathcal{X}$ triangular form, unsplit case). Let $\mathcal{X}$ be a matrix setup whose summands are arranged in decreasing order, as in (5.11.1). As usual, let $n$ denote the number of individual summands $\Gamma / \mathfrak{m}^{j}$ of $X$ each counted as often as it occurs. Recall that each column of the $n$-column matrix $A$ has a length label. Since this label sequence consists of $n$ terms, we can use it to label both the rows and columms of any $n \times n$ matrix, and we can use the noninfinite labels to label both the rows and colums of any $\left(n-e_{\infty}\right) \times\left(n-e_{\infty}\right)$ matrix. This partitions such matrices into blocks. If, for example, $Q$ is a matrix being partitioned in this way, $Q$ [rows $i$, cols $j$ ] denotes the $e_{i} \times e_{j}$ submatrix consisting of the intersection of the $i$-labeled rows with the $j$-labeled columns of $Q$.

We say that an $n \times n$ matrix $Q_{1}$ is $\mathcal{X}$ upper triangular if $Q_{1}$ [rows $i$, cols $\left.j\right]=$ 0 when $i<j$. (In interpreting this, remember that the length labels occur in decreasing order.) For example, see the matrix $Q_{1}$ in (5.12.1), noting that we have supressed multiplicities, writing each distinct length label only once. Similarly, we say that an $\left(n-e_{\infty}\right) \times\left(n-e_{\infty}\right)$ matrix $Q_{2}$ is in $\mathcal{X}$ lower triangular if $Q_{2}$ [rows $i$, cols $\left.j\right]=0$ when $i>j$. (Again see the example in (5.12.1).) Finally, we call any block of the form $Q$ [rows $i$, cols $i]$ a maindiagonal block.

$$
Q_{1}=
$$

$Q_{2}=$

\begin{tabular}{|c|c|c|c|c|c|}
\hline & $\infty$ & 6 & 4 & & \\
\hline 0 & {$\left[Q_{1}[\infty, \infty]\right.$} & $*$ & * & $6\left[\begin{array}{cc}0 \\
Q_{2}[6 . & 6\end{array}\right.$ & $\begin{array}{l}4 \\
0\end{array}$ \\
\hline 6 & 0 & $Q_{1}[6,6]$ & * & \begin{tabular}{c|c}
$02[0,0]$ \\
4 & $*$
\end{tabular} & $Q_{2}[4,4]$ \\
\hline & 0 & 0 & $Q_{1}[4,4]$ & & \\
\hline
\end{tabular}

We reformulate and prove Theorem 5.11 in the new notation.

Theorem 5.13. Let $(A, B)$ and $\left(A^{\prime}, B^{\prime}\right)$ be matrix pairs associated with a matrix setup $\mathcal{X}(\Lambda$ unsplit Dedekind-like) whose summands occur in descending order, as in (5.11.1). Then $M(A, B) \cong M\left(A^{\prime}, B^{\prime}\right)$ if and only if there exist matrix relations $A^{\prime}=P_{1} A Q_{1}$ and $B^{\prime}=P_{2} B Q_{2}$ in which the following conditions hold.

(i) $P_{1}$ and $P_{2}$ are invertible, with entries in $k$.

(ii) $Q_{1}$ is invertible and $\mathcal{X}$ upper triangular, with entries in $F$.

(iii) $Q_{2}$ is invertible and $\mathcal{X}$ lower triangular, with entries in $F$. 
(iv) $Q_{2}[$ rows $i$, cols $i]=Q_{1}[$ rows $i$, cols $i]$ whenever $i \neq \infty$; that is, every diagonal block of $Q_{2}$ equals the corresponding diagonal block of $Q_{1}$.

Remark. The matrices in (5.12.1) illustrate the triangular forms of the matrices $Q_{1}$ and $Q_{2}$ in this theorem. In addition to what is displayed in (5.12.1), the matrices in the theorem satisfy $Q_{2}[$ rows $i$, cols $i]=Q_{1}$ [rows $i$, cols $\left.i\right]$ for $i \neq \infty$.

Proof. Let $X$ be the $\Gamma$-module associated with $\mathcal{X}$. Also, let $K(A, B)$ denote the kernel of the natural homomorphism $S(A) \rightarrow M(A, B)$. The matrices $P_{i}$ and $Q_{i}$ in this theorem arise from an automorphism $\tau$ of $X$. Therefore, as in the rest of this series of papers, $\tau$ acts on the right.

Claim 1: $M(A, B) \cong M\left(A^{\prime}, B^{\prime}\right)$ if and only if there is a $\Gamma$-automorphism $\tau$ of $X$ such that $(S(A)) \tau=S\left(A^{\prime}\right)$ and $(K(A, B)) \tau=K\left(A^{\prime}, B^{\prime}\right)$ (as illustrated below).

$$
\begin{aligned}
& K(A, B) \quad \subset \quad S(A) \quad \rightarrow \quad M(A, B) \\
& (\cong) \downarrow \tau \quad(\cong) \downarrow \tau \quad(\cong) \downarrow \\
& K\left(A^{\prime}, B^{\prime}\right) \subset S\left(A^{\prime}\right) \quad \rightarrow \quad M\left(A^{\prime}, B^{\prime}\right) .
\end{aligned}
$$

First let $\sigma: M(A, B) \cong M\left(A^{\prime}, B^{\prime}\right)$ be given. Since $S(A) \rightarrow M(A, B)$ is a separated cover [Theorem 5.10(ii)], the almost functorial property of separated covers [Theorem 4.12] yields a $\Lambda$-isomorphism $\tau$ such that both squares in (5.13.1) commute. Also, since $X=\Gamma S(A)=\Gamma \otimes_{\Lambda} S(A)$ [Theorem 5.10(i)], we can extend $\tau$ to a $\Gamma$-automorphism of $X$, namely $1 \otimes \tau$. The converse assertion of the claim is obvious.

The effect of Claim 1 is that we have no further need for $M(A, B)$ and $M\left(A^{\prime}, B^{\prime}\right)$ in the rest of this proof. We replace the condition $M(A, B) \cong$ $M\left(A^{\prime}, B^{\prime}\right)$ by the condition that the automorphism $\tau$ exists.

Now suppose that the $\tau$ exists. To see how $P_{1}$ and $Q_{1}$ arise, we build the following commutative cube. Let the back (inner) square be the pullback diagram that defines $A$, and the front (outer) square be the pullback diagram that defines $A^{\prime}$. Then insert $\tau$ in the two places shown.

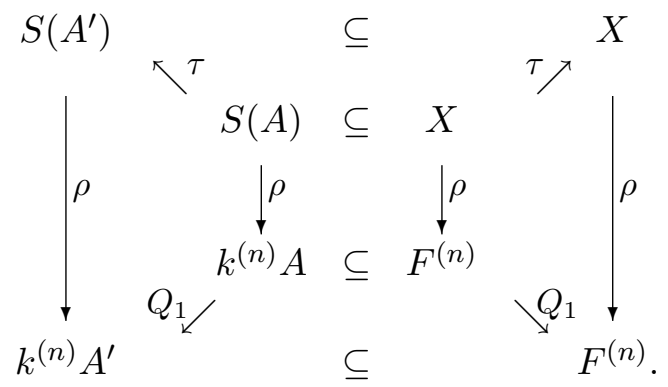

The top square commutes, by the hypothesis on $\tau$ in this half of the proof. Since $\rho$ is a surjection and $\operatorname{ker}(\rho)=\mathfrak{m} X$, there is a unique (necessarily 
invertible) matrix $Q_{1}$ over $F$ such that right multiplication by $Q_{1}$ makes the right-hand square commute. Since $\tau$ takes $S(A)$ to $S\left(A^{\prime}\right)$, and the images of $S(A)$ and $S\left(A^{\prime}\right)$ under $\rho$ are $k^{(n)} A$ and $k^{(n)} A^{\prime}$ respectively, the left-hand square is a restriction of the right-hand square, and therefore commutes.

Therefore $k^{(n)} A Q_{1}=k^{(n)} A^{\prime}$. Since $A^{\prime}$ is invertible, this can be rewritten $k^{(n)} A Q_{1}\left(A^{\prime}\right)^{-1}=k^{(n)}$. Thus right multiplication by $A Q_{1}\left(A^{\prime}\right)^{-1}$ is a $k$-linear automorphism of $k^{(n)}$, and hence equals right multiplication by a unique invertible matrix over $k$ that we call $P_{1}^{-1}$. But then $A Q_{1}\left(A^{\prime}\right)^{-1}=P_{1}^{-1}$, which is equivalent to $A^{\prime}=P_{1} A Q_{1}$, as desired.

To see how $P_{2}$ and $Q_{2}$ arise, we build the next diagram, starting with its top and bottom rows, as shown.

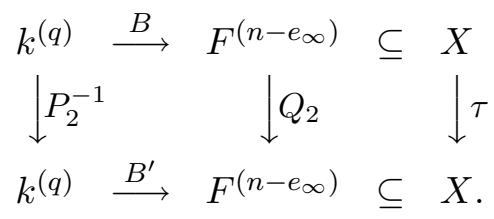

Since $F^{\left(n-e_{\infty}\right)}$ is the $\Gamma$-socle of $X$, the $\Gamma$-automorphism $\tau$ takes $F^{\left(n-e_{\infty}\right)}$ isomorphically onto itself. Therefore the restriction of $\tau$ to this submodule induces an isomorphism (given by an invertible matrix $Q_{2}$ ) from $F^{\left(n-e_{\infty}\right)}$ to $F^{\left(n-e_{\infty}\right)}$ making the right-hand square of (5.13.3) commute.

We have $(K(A, B)) \tau=K\left(A^{\prime}, B^{\prime}\right)$ by (5.13.1). Since $K(A, B)=k^{(q)} B$ and $K\left(A^{\prime}, B^{\prime}\right)=k^{(q)} B^{\prime}$, this and the second square in (5.13.3) yield $k^{(q)} B Q_{2}=$ $k^{(q)} B^{\prime}$. Since right multiplication by $B^{\prime}$ is one-to-one, this defines a one-toone $k$-linear map $k^{(q)} \rightarrow k^{(q)}$ which is therefore also surjective and equals right multiplication by a unique invertible matrix over $k$ that we call $P_{2}^{-1}$, making the left-hand square in (5.13.3) commute. Commutativity of the first square in (5.13.3) is precisely the desired relation $B^{\prime}=P_{2} B Q_{2}$.

To complete the proof of this half of the theorem we need to show that the existence of $\tau$ implies the stated block triangular forms of $Q_{1}$ and $Q_{2}$. Recall the structure of $X$ (below), where $n=\sum_{i} e_{i}$.

$$
\begin{aligned}
X & =\Gamma^{\left(e_{\infty}\right)} \oplus\left(\Gamma / \mathfrak{m}^{t}\right)^{\left(e_{t}\right)} \oplus \ldots \oplus\left(\Gamma / \mathfrak{m}^{i}\right)^{\left(e_{i}\right)} \oplus \ldots \oplus(\Gamma / \mathfrak{m})^{\left(e_{1}\right)} \\
& \downarrow \tau=\left(\tau_{\mu \nu}\right) \\
X & =\Gamma^{\left(e_{\infty}\right)} \oplus\left(\Gamma / \mathfrak{m}^{t}\right)^{\left(e_{t}\right)} \oplus \ldots \oplus\left(\Gamma / \mathfrak{m}^{i}\right)^{\left(e_{i}\right)} \oplus \ldots \oplus(\Gamma / \mathfrak{m})^{\left(e_{1}\right)} .
\end{aligned}
$$

We can view $\tau$ as right multiplication by a matrix $\left(\tau_{\mu \nu}\right)$ each of whose entries is a $\Gamma$-homomorphism

$$
\tau_{\mu \nu}: \Gamma / \mathfrak{m}^{i} \rightarrow \Gamma / \mathfrak{m}^{j} \quad(\mu \in \operatorname{block} i, \nu \in \operatorname{block} j)
$$

where " $\mu \in$ block $i$ " signifies that summand $\mu$ of $X$ is one of the $e_{i}$ summands equal to $\Gamma / \mathfrak{m}^{i}$. 
The rest of the proof uses the fact that, because $\Gamma$ is a DVR, each $\Gamma / \mathfrak{m}^{t}$ is a uniserial $\Gamma$-module, and has finite length when $t \neq \infty$. Since $\rho$ is a ring homomorphism of $\Gamma$ onto $F$ and has kernel $\mathfrak{m}$, we identify $\rho$ with the natural homomorphism $\Gamma \rightarrow \Gamma / \mathfrak{m}$. We note that, since $\tau_{\mu \nu}$ is $\Gamma$-linear, $\tau_{\mu \nu}$ equals multiplication by an element of $\Gamma$. That is, there exists $\gamma \in \Gamma$ such that, for all $x \in \Gamma$ :

$$
\left(x+\mathfrak{m}^{i}\right) \tau_{\mu \nu}=x \cdot\left(1+\mathfrak{m}^{i}\right) \tau_{\mu \nu}=x\left(\gamma+\mathfrak{m}^{j}\right) .
$$

Moreover, $\tau_{\mu \nu}$ induces the endomorphism of the $F$-vector space $F=\Gamma / \mathfrak{m}$ given by multiplication by $\rho(\gamma)=\gamma+\mathfrak{m}$. Finally, when $i \neq \infty$, the homomorphism $\tau_{\mu \nu}$ must take the unique minimal $\Gamma$-submodule $\mathfrak{m}^{i-1} / \mathfrak{m}^{i}$ (our standard copy of $F$ ) to a module of length at most 1 . We break up the remaining details into three cases.

Case 1: $i<j$ (below the main-diagonal blocks). Here $\Gamma / \mathfrak{m}^{i}$ is uniserial of finite length $i$ and hence its image under $\tau_{\mu \nu}$ is uniserial of length at most $i$. Therefore $\operatorname{im}\left(\tau_{\mu \nu}\right) \subseteq \mathfrak{m} / \mathfrak{m}^{j}$, and so the induced map $\Gamma / \mathfrak{m} \rightarrow \Gamma / \mathfrak{m}$ equals zero. In other words, the $(\mu, \nu)$-entry of $Q_{1}$ is zero whenever $(\mu, \nu)$ belongs to a block $Q_{1}$ [rows $i$, cols $\left.j\right]$ that lies below the main-diagonal blocks, as claimed in statement (ii).

Case 2: $\infty \neq i>j$ (above the main-diagonal blocks). Here $\tau_{\mu \nu}$ cannot be one-to-one, and hence takes the unique simple $\Gamma$-submodule $\mathfrak{m}^{i-1} / \mathfrak{m}^{i}$ of $\Gamma / \mathfrak{m}^{i}$ to zero. Therefore, in this case, the $(\mu, \nu)$-entry of $Q_{2}$ equals zero as claimed in (iii).

Case 3: $i=j \neq \infty$ (main-diagonal block). We have already noted that the endomorphism of $\Gamma / \mathfrak{m}$ induced by $\tau_{\mu \nu}$ equals multiplication by $\rho(\gamma)$. But since $\tau_{\mu \nu}$ equals multiplication by $\gamma \in \Gamma$ on $\Lambda / \mathfrak{m}^{i}$, it follows that $\tau_{\mu \nu}$ induces an endomorphism of the socle $\mathfrak{m}^{i-1} / \mathfrak{m}^{i}$ of $\Gamma / \mathfrak{m}$, again given by multiplication by the same element $\rho(\gamma)$, as desired.

This completes the proof of the "only if" part of the theorem.

Conversely, suppose that $A^{\prime}=P_{1} A Q_{1}$ and $B^{\prime}=P_{2} B Q_{2}$ and (i)-(iv) hold. We want to prove that a $\Gamma$-automorphism $\tau$ of $X$ exists satisfying Claim 1, that is, such that $(S(A)) \tau=S\left(A^{\prime}\right)$ and $(K(A, B)) \tau=K\left(A^{\prime}, B^{\prime}\right)$. We define the map $\tau$ by defining each $\tau_{\mu \nu}$ in (5.13.5), considering two cases.

Case A. $i \geq j$ (on or above main diagonal). Let $x$ be the $(\mu, \nu)$-entry of $Q_{1}$. Then $x=\rho(\gamma)$ for some $\gamma \in \Gamma$. Since $i \geq j$, multiplication by $\gamma$ followed by reduction modulo $\mathfrak{m}^{j}$ is a well-defined $\Gamma$-homomorphism that we use for $\tau_{\mu \nu}$ in (5.13.5).

Case B. $i<j$ (below main diagonal). Using our standard $\Gamma$-generator $\pi$ of $\mathfrak{m}$, multiplication by $\pi^{j-i}$ yields a $\Gamma$-embedding of $\Gamma / \mathfrak{m}^{i}$ into $\Gamma / \mathfrak{m}^{j}$. Let $x$ be the $(\mu, \nu)$-entry of $Q_{2}$, and let $\gamma$ be any element of $\Gamma$ such that $\rho(\gamma)=x$. 
Then multiplication by $\pi^{j-i} \cdot \gamma$ is a well-defined $\Gamma$-linear map that we use for $\tau_{\mu \nu}$ in (5.13.5).

Claim 2: With the given definition of $\tau$, the right-hand square in diagram (5.13.2) commutes. It suffices to check commutativity of each smaller square, in which $\tau$ is replaced by some $\tau_{\mu \nu}$ in (5.13.5) and $Q_{1}$ is replaced by its $(\mu, \nu)$-entry. Except if the block containing $(\mu, \nu)$ is strictly below the maindiagonal blocks, this is true by the definition in Case A. For the belowdiagonal blocks, the map induced by $\tau_{\mu \nu}$ is zero regardless of our choice of $\tau_{\mu \nu}$, as shown in the proof of Case 1 above; so the claim, for below-diagonal blocks, follows from the block upper triangular form of $Q_{1}$.

Claim 3: The endomorphism $\tau$ is an automorphism. The map induced by $\tau$ modulo $\mathfrak{m}$ - i.e., right multiplication by $Q_{1}$ - is a surjection. Therefore, by Nakayama's lemma, $\tau$ itself is a surjection. Since $X$ is noetherian, it follows that $\tau$ is also an injection.

Claim 4: The endomorphism $\tau$ induces right multiplication by $Q_{2}$; that is, the right-hand square of diagram (5.13.3) commutes. As in the proof of Claim 2, it suffices to check each $\tau_{\mu \nu}$ individually. For $(\mu, \nu)$ strictly below the main-diagonal blocks this is true by the definition in Case B. For $(\mu, \nu)$ strictly above the main-diagonal blocks, the map induced by $\tau_{\mu \nu}$ is zero regardless of our choice of $\tau_{\mu \nu}$, as shown in the proof of Case 2 above. This leaves only the case that $(\mu, \nu)$ belongs to some main-diagonal block, say [rows $i$, cols $i$ ].

Let $\gamma$ and $x=\rho(\gamma)$ be as in Case A of our definition. Then $x$ is the $(\mu, \nu)$ entry of $Q_{1}$, and hence, by condition (iv) in the statement of the theorem, $x$ is the $(\mu, \nu)$-entry of $Q_{2}$. The desired commutativity of the second square of diagram (5.13.3) is now immediate.

Now we are ready to prove that $(S(A)) \tau=S\left(A^{\prime}\right)$. First note that, in the bottom left of diagram (5.13.2), right multiplication by $Q_{1}$ takes $k^{(n)} A$ onto $k^{(n)} A^{\prime}$ because $A^{\prime}=P_{1} A Q_{1}$ and $P_{1}$ is an invertible matrix over $k$. Therefore the bottom square commutes. The inner and outer squares commute, being pullback squares by definition (5.8.3), and the right-hand square commutes by Claim 2. Therefore the upper left map $\tau$ takes the pullback $S(A)$ of the inner square onto the pullback $S\left(A^{\prime}\right)$ of the outer square, as claimed.

Finally, we show that $(K(A, B)) \tau=K\left(A^{\prime}, B^{\prime}\right)$. The left-hand square in (5.13.3) commutes since $B^{\prime}=P_{2} B Q_{2}$, and the right-hand square commutes by Claim 4. Therefore $\tau$ takes $\operatorname{im}(B)=K(A, B)$ onto $\operatorname{im}\left(B^{\prime}\right)=K\left(A^{\prime}, B^{\prime}\right)$.

Notation 5.14. Let $(A, B)$ and $\left(A^{\prime}, B^{\prime}\right)$ be matrix pairs associated with matrix setups $\mathcal{X}$ and $\mathcal{X}^{\prime}$ respectively (unsplit case) [Notation 5.7]. We write $(A, B) \cong\left(A^{\prime}, B^{\prime}\right)$ to indicate that each pair can be obtained from the other 
by means of display operations [Definition 5.9] and $k-F$ sweeping-similarity operations [Theorem 5.11].

It is easy to see that, when such an isomorphism holds, we can do the display operations before the sweeping-similarity operations. After doing the display operations and changing notation, we have $\mathcal{X}=\mathcal{X}^{\prime}$. Moreover, when $\mathcal{X}=\mathcal{X}^{\prime}$ we have $(A, B) \cong\left(A^{\prime}, B^{\prime}\right)$ if and only if each pair can be obtained from the other by $k-F$ sweeping-similarity alone.

The following result summarizes much of the content of this section.

Theorem 5.15. In the unsplit case, every finitely generated $\Lambda$-module is isomorphic to some $M(A, B)$ associated with some matrix setup. Moreover, $M(A, B) \cong M\left(A^{\prime}, B^{\prime}\right)$ if and only if $(A, B) \cong\left(A^{\prime}, B^{\prime}\right)$.

Proof. The first assertion is Theorem 5.10(iii). Next, suppose that $M(A, B)$ $\cong M\left(A^{\prime}, B^{\prime}\right)$. Then $X \cong X^{\prime}$, where $X$ and $X^{\prime}$ are the $\Gamma$-modules associated with the matrix setups given by the row of length labels in $(A, B)$ and in $\left(A^{\prime}, B^{\prime}\right)$ [Theorem 5.10(iv)].

Consider the decomposition (5.6.2) of $X$, whose summands are various modules $\Gamma / \mathfrak{m}^{t}(1 \leq t \leq \infty)$, and its analog for $X^{\prime}$, which we call $(5.6 .2)^{\prime}$. For each $t$, let $X(t)$ denote the subsum, in the decomposition of $X$, consisting of all summands $\Gamma / \mathfrak{m}^{t}$. Since the Krull-Schmidt Theorem holds for finitely generated modules over principal ideal domains, $X$ and $X^{\prime}$ have the same summands $\Gamma / \mathfrak{m}^{t}$, except for order of occurrence. In particular, $X(t)=X^{\prime}(t)$ for every $t$. Therefore some permutation of the indecomposable summands of $X$ transforms them to the indecomposable summands of $X^{\prime}$.

This permutation of summands in (5.6.2) and (5.6.2) defines a display operation on $(A, B)$. After performing this display operation we have $X=$ $X^{\prime}$, whence Theorem 5.11 states that $k-F$ sweeping-similarity operations transform $(A, B)$ to $\left(A^{\prime}, B^{\prime}\right)$, as desired.

The converse implication holds because neither display operations [(5.9.1)] nor sweeping-similarity operations [Theorem 5.11] change the isomorphism class of $M(A, B)$.

Reminder. This is a good point to remind readers of the existence of the terminological index in $\S 13$.

\section{Isomorphism as matrix problem, strictly split case.}

The main results of this section use separated covers to transform the problem of describing isomorphism classes of $\Lambda$-modules into a matrix problem, for the case that $\Lambda$ is a strictly split Dedekind-like ring. Since this problem was solved in $[\mathbf{L 1}, \mathbf{L 3}]$ (and in $[\mathbf{N R}]$, corrected in $[\mathbf{N R S B}]$ ), we omit most details, which are very similar to the corresponding details in the unsplit case considered in the previous section. The emphasis here is on phrasing these results in the terminology of the present paper, in such a way that 
it will be useful later in this paper and in the forthcoming paper on the nonlocal situation.

The principal differences between results in the split and unsplit cases are due to the fact that, in the split case, $\Gamma$ is the direct sum of two DVRs, while in the unsplit case, $\Gamma$ is a single DVR. Therefore, in the split case, there are twice as many matrices to manipulate as in the unsplit case. But in the split case only one field is involved, namely $k$.

Notation 6.1. Throughout this section $(\Lambda, \mathfrak{m}, k)$ is a strictly split Dedekindlike ring, as in Notation 1.1. Thus the normalization of $\Lambda$ is $\Gamma=\Gamma_{1} \oplus \Gamma_{2}$ where each $\left(\Gamma_{i}, \mathfrak{m}_{i}, k\right)$ is a DVR and $\mathfrak{m}=\mathfrak{m}_{1} \oplus \mathfrak{m}_{2}$. Following the style of Notation 5.6, we use the notation $\rho$ not only for the map $\Gamma \rightarrow \bar{\Gamma}=\Gamma / \mathfrak{m}=k \oplus k$ in pullback diagram (1.1.1), but also for the maps $\Gamma_{i} / \mathfrak{m}_{i}^{t} \rightarrow k$ induced by the original map $\rho$, and for direct sums of such maps. Moreover, we let $\mathfrak{m}_{i}^{\infty}=0$, so that $\Gamma_{i}=\Gamma_{i} / \mathfrak{m}_{i}^{\infty}$.

Example 6.2. Note that the two direct summands $k$ of $\bar{\Gamma}$ are isomorphic rings but not isomorphic $\Gamma$-modules (because their annihilators are different). Thus the field $k$ has two $\Gamma$-module structures. When necessary we distinguish between these nonisomorphic $\Gamma$-modules by using the notation $(k, 0)$ and $(0, k)$. However - and this is very important in the rest of this paper — we have $(k, 0) \cong(0, k)$ as $\Lambda$-modules (equivalently, as $k$-vector spaces).

Let $X$ be any of the nonisomorphic $\Gamma$-modules $(k, 0),(0, k),(k, k)=$ $(k, 0) \oplus(0, k)$. Each of these has a $\Lambda$-submodule $S$ that is $\Lambda$-isomorphic to $k$ and such that $X=\Gamma S$, namely $X$ itself in the first two cases, and the diagonal submodule $\operatorname{diag}(k)=\{(x, x) \in k \oplus k\}$ in the third case. However only $S=\operatorname{diag}(k)$ satisfies $\Gamma S=\Gamma \otimes_{\Lambda} S$ [Lemma 5.2].

This shows that the notation $\Gamma S$ is not well-defined unless one specifies the $\Gamma$-module $X$ inside of which it is computed.

Notation 6.3 (Matrix setup, strictly split case). Let $t \neq \infty$. Then, making the identifications $k=\Gamma_{i} / \mathfrak{m}_{i}(i=1,2)$ via $\rho$, we have $k \cong \mathfrak{m}_{i}^{t-1} / \mathfrak{m}_{i}^{t}$ $(i=1,2)$ as $k$-vector spaces via $\gamma_{i}+\mathfrak{m}_{i} \rightarrow \gamma_{i} \pi_{i}^{t-1}+\mathfrak{m}_{i}^{t}$, where $\pi_{i}$ is the standard $\Gamma$-generator of $\mathfrak{m}_{i}$ mentioned in Notation 1.1. Thus every element of $k$ has a standard image in each $\mathfrak{m}_{i}^{t-1} / \mathfrak{m}_{i}^{t}$, which we often regard as an identification; and the set of all such standard images defines the standard copy of $k$ in $\Gamma_{i} / \mathfrak{m}_{i}^{t}$.

Let $X$ be the nonzero (external) direct sum of $\Gamma$-modules displayed below.

$$
\begin{gathered}
X=X_{1} \oplus X_{2} \quad \text { where } \\
X_{1}=\oplus_{\nu=1}^{n} \Gamma_{1} / \mathfrak{m}_{1}^{s_{\nu}} \quad \text { and } \quad X_{2}=\oplus_{\nu=1}^{n} \Gamma_{2} / \mathfrak{m}_{2}^{t_{\nu}}
\end{gathered}
$$

where each $s_{\nu}$ and $t_{\nu}$ is a positive integer or $\infty$. Since each $\Gamma_{i}$ is a DVR, every finitely generated $\Gamma_{i}$-module is isomorphic to a module of the form $X_{i}(i=$ 
$1,2)$. However, note that the same number $n$ of indecomposable summands occurs in both $X_{1}$ and $X_{2}$ in (6.3.1). The reason for this restriction is the following fact, which we will have many occasions to use.

$$
\text { The } \Gamma \text {-module } Y=\left(\oplus_{\nu=1}^{m} \Gamma_{1} / \mathfrak{m}_{1}^{s_{\nu}}\right) \oplus\left(\oplus_{\nu=1}^{n} \Gamma_{2} / \mathfrak{m}_{2}^{t_{\nu}}\right) \text { has a } \Lambda \text { - }
$$
submodule $S$ such that $Y=\Gamma \otimes_{\Lambda} S$ if and only if $m=n$.

This follows immediately from Corollary 5.4 since - in the notation of Example 6.2 - the right hand side, when reduced modulo $\mathfrak{m}=\mathfrak{m}_{1} \oplus \mathfrak{m}_{2}$, becomes $\Gamma$-isomorphic to $(k, 0)^{(m)} \oplus(0, k)^{(n)}$, while $\Gamma / \mathfrak{m} \cong(k, k)$.

The map $\rho$ yields a pair of $\Gamma_{i}$-linear surjections to $k^{(n)}$ shown in the first part of (6.3.3).

$$
\rho: X_{i} \rightarrow k^{(n)} \quad(i=1,2)
$$$$
k^{\left(n-d_{\infty}\right)} \subseteq X_{1}
$$$$
k^{\left(n-e_{\infty}\right)} \subseteq X_{2}
$$

where $k$ really denotes $(k, 0)$ when $i=1$, and $(0, k)$ when $i=2$. We call coordinate $\nu$ of $k^{(n)}$ the coordinate corresponding to the the $\nu^{\text {th }}$ summand $\Gamma_{1} / \mathfrak{m}_{1}^{s_{\nu}}$ of $X_{1}$. Then an analogous definition applies to $X_{2}$.

Let $d_{\infty}, e_{\infty}$, respectively, be the number of indecomposable summands of $X_{1}, X_{2}$ of infinite length. Each of the $n-d_{\infty}$ summands of finite length contains a standard copy of $k$. This yields the first inclusion shown in (6.3.3). Thus every coordinate of $k^{\left(n-d_{\infty}\right)}$ has a corresponding summand $\Gamma_{1} / \mathfrak{m}_{1}^{s_{\nu}}$ of $X_{1}$, namely the summand containing this copy of $k$. Analogous statements apply to the second displayed inclusion.

A matrix setup $\mathcal{X}$ (for $\Lambda$ strictly split Dedekind-like) is any three directsum decompositions of the form (6.3.1), together with the corresponding surjections and inclusions displayed in (6.3.3). The $\Gamma$-module associated with $\mathcal{X}$ is the module $X$ in (6.3.1). (This replaces the somewhat differently stated, but equivalent, "matrizing choices" in $[\mathbf{L} 1, \mathbf{L} 3]$.

Notation 6.4 (Matrix 4-tuple $\left(A_{1}, A_{2}, B_{1}, B_{2}\right)$; associated $\Lambda$-module). Let $\mathcal{X}$ be a matrix setup (strictly split case). Let $A_{1}$ and $A_{2}$ be $n \times n$ matrices over $k$, and let $B_{1}$ and $B_{2}$ be $q \times\left(n-d_{\infty}\right)$ and $q \times\left(n-e_{\infty}\right)$ matrices, respectively, over $k$ (for some $q \geq 0$ ).

We attach a length label to each column of these matrices as in the unsplit case: Define the length label of column $\nu$ of $A_{1}$ to be the length $s_{\nu}$ of its corresponding direct summand $\Gamma_{1} / \mathfrak{m}^{s_{\nu}}$ of $X_{1}$, and define the length label of each column of $B_{1}$ to be the length of its corresponding summand of $X_{1}$. Thus every column of $B_{1}$ has a corresponding column in $A_{1}$, namely the column with the same corresponding summand (of finite length) of $X_{1}$. Analogous definitions apply to $A_{2}, B_{2}, X_{2}$.

We require these matrices to satisfy:

(6.4.1) $A_{1}$ and $A_{2}$ are invertible; the rows of each $B_{i}$ are linearly independent over $k$; and the columns of each $B_{i}$ with length label 1 consist of zeros. 
Consequently, right multiplication by $\left(A_{1}, A_{2}\right)$ and by $\left(B_{1}, B_{2}\right)$ are $k$-linear monomorphisms:

$$
\left(A_{1}, A_{2}\right): k^{(n)} \hookrightarrow k^{(n)} \oplus k^{(n)} \quad\left(B_{1}, B_{2}\right): k^{(q)} \hookrightarrow k^{\left(n-d_{\infty}\right)} \oplus k^{\left(n-e_{\infty}\right)} .
$$

We call $\left(A_{1}, A_{2}, B_{1}, B_{2}\right)$ a matrix 4 -tuple associated with $\mathcal{X}$.

As in the unsplit case, we write these four matrices side by side, but we always think of each $B_{i}$ as being written underneath $A_{i}$, with each column of $B_{i}$ written under the corresponding column of $A_{i}$ (and no column of $B_{i}$ written under columns of $A_{i}$ with infinite length labels). (See (7.1.1) for an example of a 4 -tuple written out in this form.)

Using the maps in (6.4.2), let $S\left(A_{1}, A_{2}\right)$ be the $\Lambda$-module

$$
S\left(A_{1}, A_{2}\right)=\left\{x \in X \mid \rho(x) \in \operatorname{im}\left(A_{1}, A_{2}\right)=k^{(n)} \cdot\left(A_{1}, A_{2}\right)\right\}
$$

and define the $\Lambda$-module $M\left(A_{1}, A_{2}, B_{1}, B_{2}\right)$ by

$$
\begin{gathered}
M\left(A_{1}, A_{2}, B_{1}, B_{2}\right)=S\left(A_{1}, A_{2}\right) / \operatorname{im}\left(B_{1}, B_{2}\right) \\
\text { where } \operatorname{im}\left(B_{1}, B_{2}\right)=k^{(q)} \cdot\left(B_{1}, B_{2}\right) .
\end{gathered}
$$

If $q=0$ we interpret this to mean that $M\left(A_{1}, A_{2}, B_{1}, B_{2}\right)=S\left(A_{1}, A_{2}\right)$. We call $M\left(A_{1}, A_{2}, B_{1}, B_{2}\right)$ the $\Lambda$-module associated with $\left(A_{1}, A_{2}, B_{1}, B_{2}\right)$ (with respect to the matrix setup $\mathcal{X})$.

We note that $\Gamma \cdot S\left(A_{1}, A_{2}\right)=X$, since $A_{1}$ and $A_{2}$ are invertible. Thus $S\left(A_{1}, A_{2}\right)$ is the pullback of the commutative square in diagram (6.4.5) below.

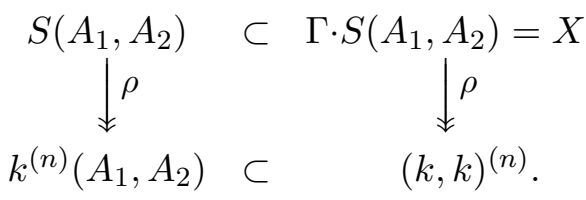

The similarity between (6.4.5) and (5.8.3) can be enhanced by noting that we can consider the $n \times 2 n$ matrix $\left(A_{1}, A_{2}\right)$ over $k$ to be an invertible $n \times n$ matrix over $\bar{\Gamma}=k \oplus k$, and just calling it $A$. Although this is sometimes a helpful point of view, it will more often be useful to focus on the individual matrices $A_{1}$ and $A_{2}$. However, we cannot consider $\left(B_{1}, B_{2}\right)$ to be a matrix over $\bar{\Gamma}$ because the number of columns of $B_{1}$ need not equal the number of columns of $B_{2}$.

Definition 6.5 (Display operation, split case). We define a display operation on a matrix 4 -tuple $\left(A_{1}, A_{2}, B_{1}, B_{2}\right)$ (associated with a matrix setup $\mathcal{X})$ to be a permutation of the columns of each $A_{i}$, together with the same permutation of their length labels and corresponding columns of $B_{i}$ (so that corresponding columns of $A_{i}$ and $B_{i}$ remain corresponding columns after being moved). 
Thus each display operation corresponds to the effect on $\left(A_{1}, A_{2}, B_{1}, B_{2}\right)$ of rearranging the summands $\Gamma_{i} / \mathfrak{m}_{i}^{u}$ in decompositions (6.3.1) of each $X_{i}$. For future reference we record the following consequence of this:

(6.5.1) Let $\left(A_{1}^{\prime}, A_{2}^{\prime}, B_{1}^{\prime}, B_{2}^{\prime}\right)$ — including its length labels — be obtained by performing a display operation on $\left(A_{1}, A_{2}, B_{1}, B_{2}\right)$. Then $M\left(A_{1}^{\prime}, A_{2}^{\prime}\right.$, $\left.B_{1}^{\prime}, B_{2}^{\prime}\right) \cong M\left(A_{1}, A_{2}, B_{1}, B_{2}\right)$, where the module on the left is computed with respect to the correspondingly altered matrix setup.

We will need display operations to display our matrix pairs in canonical form.

Theorem 6.6. Let $\mathcal{X}$ be a matrix setup (strictly split case) with associated $\Gamma$-module $X$, and let $\left(A_{1}, A_{2}, B_{1}, B_{2}\right)$ be a matrix 4-tuple associated with $\mathcal{X}$. Also, let $S=S\left(A_{1}, A_{2}\right)$. Then:

(i) $\Gamma S=\Gamma \otimes_{\Lambda} S$.

(ii) The natural surjection $S \rightarrow M\left(A_{1}, A_{2}, B_{1}, B_{2}\right)$ is a separated cover. Moreover,

(iii) Every finitely generated $\Lambda$-module is isomorphic to $M\left(A_{1}, A_{2}, B_{1}, B_{2}\right)$ for some matrix 4-tuple $\left(A_{1}, A_{2}, B_{1}, B_{2}\right)$ with respect to some matrix setup $\mathcal{X}$.

(iv) Let $X$ and $X^{\prime}$ be the $\Gamma$-modules associated with matrix setups $\mathcal{X}$ and $\mathcal{X}^{\prime}$ respectively, and let $M\left(A_{1}, A_{2}, B_{1}, B_{2}\right)$ and $M\left(A_{1}^{\prime}, A_{2}^{\prime}, B_{1}^{\prime}, B_{2}^{\prime}\right)$ be $\Lambda$ modules associated with $\mathcal{X}$ and $\mathcal{X}^{\prime}$ respectively. If $M\left(A_{1}, A_{2}, B_{1}, B_{2}\right) \cong$ $M\left(A_{1}^{\prime}, A_{2}^{\prime}, B_{1}^{\prime}, B_{2}^{\prime}\right)$ as $\Lambda$-modules then $X \cong X^{\prime}$ as $\Gamma$-modules.

Proof. The proof is a minor modification of that of Theorem 5.10, so we omit most details except where the two situations differ.

(i) Use Lemma 5.2, remembering that $\operatorname{ker} \rho=\mathfrak{m} S=\mathfrak{m} X$.

(ii) The proof of Theorem 5.10(ii) works in the current context except for the proof of statement (a): $\operatorname{im}\left(B_{1}, B_{2}\right)$ contains no nonzero $\bar{\Gamma}$-submodules. If $(x, y)$ is an element of any $\bar{\Gamma}$-module, then that module also contains $(x, 0)$ and $(0, y)$ (since $\bar{\Gamma}=k \oplus k)$. But since each of $B_{1}$ and $B_{2}$ has linearly independent rows, right multiplication by these matrices is a one-to-one map. Therefore $(x, 0) \in \operatorname{im}\left(B_{1}, B_{2}\right)$ implies $x=0$, and the analogous statement holds for $(0, y)$.

(iii) Let $M$ be a finitely generated $\Lambda$-module. As in the proof of Theorem 5.10(iii) we take a separated cover $\phi: S \rightarrow M$, where $S$ is a $\Lambda$-submodule of $X:=\Gamma S=\Gamma \otimes_{\Lambda} S$. We need to prove that $S=S\left(A_{1}, A_{2}\right)$ and $\operatorname{ker}(\phi)=\operatorname{im}\left(B_{1}, B_{2}\right)$ for appropriate matrices $A_{1}, A_{2}, B_{1}, B_{2}$. In fact, we do this with $A_{1}$ and $A_{2}$ equal to identity matrices. (However, we will not always have each $A_{i}=I$, later in this paper.)

Since the $\Lambda$-module $S$ is finitely generated [Proposition 4.11], so is the $\Gamma$-module $X=\Gamma \otimes_{\Lambda} S$. Since $\Gamma_{1}, \Gamma_{2}$ are DVRs, we can take $X$ to be as 
displayed as in (6.3.1), provided that we can show that $X_{1}$ and $X_{2}$ have the same number of indecomposable direct summands. This is done in (6.3.2). The inclusion $S \subset X$ gives us modules to use in the top row of pullback square (6.4.5) that we are building. We will soon attach matrices to $S$.

For some $n$ we have $S / \mathfrak{m} S \cong k^{(n)}$ and $X / \mathfrak{m} X \cong \bar{\Gamma}^{(n)}$ as $\Lambda$ - and $\Gamma$-modules, respectively, by Lemma 5.2. We use the inclusion map $k^{(n)} \subset \bar{\Gamma}^{(n)}$ for the bottom row. Define the right hand vertical map to be our usual map $\rho$, and define the left-hand vertical map to be the restriction of $\rho$ to $S$. Since $\mathfrak{m} S=\mathfrak{m} \Gamma S=\mathfrak{m} X$, we have that $S$ is the pullback of this diagram and, in fact, $S=S\left(I_{n}, I_{n}\right)$ as desired.

Now we show that $\operatorname{ker}(\phi)$ has the required form, $\operatorname{im}\left(B_{1}, B_{2}\right)$. By Lemma $4.9, \operatorname{ker}(\phi)$ is a $k$-module. Choose a $k$-linear identification $\operatorname{ker}(\phi)=k^{(q)}$, for some $q$. The projection of $\operatorname{ker}(\phi)$ in each $X_{i}(i=1,2)$ is again a $k$ submodule of $X_{i}$. Therefore the projection of $\operatorname{ker}(\phi)$ in $X_{1}$ is contained in $(k, 0)^{\left(n-d_{\infty}\right)}$. Moreover, Lemma 4.9 together with the fact that we are in the strictly split case yields $\operatorname{ker}(\phi) \subseteq \mathfrak{m} S=\mathfrak{m} X=\mathfrak{m}_{1} X_{1} \oplus \mathfrak{m}_{2} X_{2}$. Since $\mathfrak{m}_{1}$ annihilates the $\Gamma_{1}$-module $\Gamma_{1} / \mathfrak{m}_{1}$, we see that the projection of $\operatorname{ker}(\phi)$ in any indecomposable summand of $X_{1}$ of length 1 is zero. An analogous statement, with $d$ replaced by $e$, holds for the projection in $X_{2}$. This gives us two $q$ rowed matrices $B_{1}, B_{2}$ over $k$ such that $\operatorname{ker}(\phi)=k^{(q)}\left(B_{1}, B_{2}\right)$, and every column with length label 1 consists of zeros. Moreover, right multiplication by $\left(B_{1}, B_{2}\right)$ from $k^{(q)}$ to $\operatorname{ker}(\phi)$ is one-to-one.

All that remains to be shown now is that each of these matrices has linearly independent rows; that is, right multipication by each of $B_{1}$ and $B_{2}$ is one-to-one. Suppose that (say) $x B_{1}=0$. Then $x \cdot\left(B_{1}, B_{2}\right)=\left(0, x \cdot B_{2}\right)$ is contained in the $\Gamma$-socle of $X_{2}$. Note that every $\Lambda$-submodule of $X_{2}$ is a $\Gamma$-module. Therefore the $\Lambda$-module $k x \cdot\left(B_{1}, B_{2}\right)=\left(0, x \cdot B_{2}\right)$ of $\operatorname{ker}(\phi)$ is a $\Gamma$-submodule. By Lemma 4.9, it follows that $x \cdot\left(B_{1}, B_{2}\right)=(0,0)$. Since right multiplication by $\left(B_{1}, B_{2}\right)$ is one-to-one, we now have $x=0$, as desired. This completes the proof of (iii).

(iv) This is exactly the same as the proof of Theorem 5.10(iv).

The final main result of this section determines when $M\left(A_{1}, A_{2}, B_{1}, B_{2}\right) \cong$ $M\left(A_{1}^{\prime}, A_{2}^{\prime}, B_{1}^{\prime}, B_{2}^{\prime}\right)$ in terms of the matrices involved. Because of Theorem 6.6(iv) we can restrict our attention to the situation that both matrices arise from the same associated matrix setup.

Theorem 6.7 (Matrix Operations, strictly split case). Suppose that ( $A_{1}$, $\left.A_{2}, B_{1}, B_{2}\right)$ and $\left(A_{1}^{\prime}, A_{2}^{\prime}, B_{1}^{\prime}, B_{2}^{\prime}\right)$ are matrix 4 -tuples over $k$ associated with the matrix setup $\mathcal{X}$ (where $\Lambda$ is strictly split Dedekind-like with residue field $k)$. Then $M\left(A_{1}, A_{2}, B_{1}, B_{2}\right) \cong M\left(A_{1}^{\prime}, A_{2}^{\prime}, B_{1}^{\prime}, B_{2}^{\prime}\right)$ if and only if $\left(A_{1}^{\prime}, A_{2}^{\prime}, B_{1}^{\prime}\right.$, $\left.B_{2}^{\prime}\right)$ can be obtained from $\left(A_{1}, A_{2}, B_{1}, B_{2}\right)$ by a finite sequence of the following operations. 
(i) (a) Simultaneously left multiply $A_{1}$ and $A_{2}$ by an invertible matrix over $k$.

(b) Simultaneously left multiply $B_{1}$ and $B_{2}$ by an invertible matrix over $k$.

(ii) (a) For any length label $j$ right-multiply $A_{1}[$ cols $j]$ by an invertible matrix $Q$ over $k$ and, if $j \neq \infty$, simultaneously right-multiply $B_{1}$ [cols $\left.j\right]$ by $Q$.

(b) For any length label $j$ right-multiply $A_{2}[\operatorname{cols} j]$ by an invertible matrix $Q$ over $k$ and, if $j \neq \infty$, simultaneously right-multiply $B_{2}[$ cols $j]$ by $Q$.

(iii) (a) For any $i>j$ add a $k$-scalar multiple of an $i$-labeled column of $A_{1}$ to a j-labeled column of $A_{1}$. ("Sweep toward smaller lengths in $\left.A_{1} \cdot "\right)$

(b) For any $i>j$ add a $k$-scalar multiple of an i-labeled column of $A_{2}$ to a $j$-labeled column of $A_{2}$. ("Sweep toward smaller lengths in $\left.A_{2} . "\right)$

(c) For any $i<j$ add a $k$-scalar multiple of an $i$-labeled column of $B_{1}$ to a $j$-labeled column of $B_{1}$. ("Sweep toward larger lengths in $B_{1}$.")

(d) For any $i<j$ add a $k$-scalar multiple of an $i$-labeled column of $B_{2}$ to a j-labeled column of $B_{2}$. ("Sweep toward larger lengths in $B_{2}$. ")

We call these matrix operations " $k$ - $k$ sweeping-similarity" operations.

Proof. We omit the proof, which is similar to the proof of Theorem 5.11 and is given in detail in [L3, Theorems 4.6,4.7], albeit it with many changes of notation. (See the next remark.)

Remark 6.8 (Comparison with notation in [L3]). The translation of isomorphism of modules to a matrix problem is carried out in [L3, §4] for commutative rings called "Dedekind-like." In the local case, this is what we call "split Dedekind-like" in [KL1], and is slightly more general than what we call "strictly split Dedekind-like" in the present paper. But in the complete local case, the two are the same. Many of the difficulties one has in reading [L3] stem from the fact that the complete local case was not completely detached from the global case, as in the present series of papers.

The four matrices that we call $A_{1}, A_{2}, B_{1}, B_{2}$ are called $C^{-1}, D^{-1}, A, B$ respectively in $[\mathbf{L} 3]$, and matrices act via left multiplication there, rather than via right multiplication, as in the present paper. Instead of making the direct module-versus-matrix connection, as done in the present paper, [L3] first connects a given module with a diagram $\mathcal{D}$ - not the same thing that is called a diagram in the present paper - that gives a detailed picture of the separated cover (called a "separated representation" there) of the module $[\mathbf{L 3}, \S 2]$ and then converts isomorphism of diagrams to a matrix problem $[\mathbf{L 3}, \S 4]$. The nature of the matrices in a matrix setup is given in [L3, Proposition 4.5], and our present Matrix Operations Theorem 6.7 is 
given in $[\mathbf{L} 3,4.6,4.7]$. In the strictly split local case, the case considered in the present paper, one can ignore the "global condition" in [L3, 4.7] since it becomes trivial locally. We return to this global condition, in greater generality, in [KL4].

Notation 6.9. Let $\left(A_{1}, A_{2}, B_{1}, B_{2}\right)$ and $\left(A_{1}^{\prime}, A_{2}^{\prime}, B_{1}^{\prime}, B_{2}^{\prime}\right)$ be matrix 4-tuples associated with matrix setups $\mathcal{X}$ and $\mathcal{X}^{\prime}$ respectively (split case) [Notation 6.4]. We write $\left(A_{1}, A_{2}, B_{1}, B_{2}\right) \cong\left(A_{1}^{\prime}, A_{2}^{\prime}, B_{1}^{\prime}, B_{2}^{\prime}\right)$ to indicate that each 4-tuple can be obtained from the other by means of display operations [Definition 6.5] and $k$ - $k$ sweeping-similarity operations [Theorem 6.7].

It is easy to see that, when such an isomorphism holds, we can do the display operations before the sweeping-similarity operations. After doing the display operations and changing notation, we have $\mathcal{X}=\mathcal{X}^{\prime}$. Moreover, when $\mathcal{X}=\mathcal{X}^{\prime}$, we have $\left(A_{1}, A_{2}, B_{1}, B_{2}\right) \cong\left(A_{1}^{\prime}, A_{2}^{\prime}, B_{1}^{\prime}, B_{2}^{\prime}\right)$ if and only if each 4 -tuple can be obtained from the other by $k$ - $k$ sweeping-similarity alone.

As in the unsplit case [Theorem 5.15], one proves:

Theorem 6.10. In the strictly split case, every finitely generated $\Lambda$-module is isomorphic to some $M\left(A_{1}, A_{2}, B_{1}, B_{2}\right)$ associated with some matrix setup. Moreover, $M\left(A_{1}, A_{2}, B_{1}, B_{2}\right) \cong M\left(A_{1}^{\prime}, A_{2}^{\prime}, B_{1}^{\prime}, B_{2}^{\prime}\right)$ if and only if $\left(A_{1}, A_{2}, B_{1}\right.$, $\left.B_{2}\right) \cong\left(A_{1}^{\prime}, A_{2}^{\prime}, B_{1}^{\prime}, B_{2}^{\prime}\right)$.

\section{Solution of matrix problem, strictly split case.}

In this section we describe the canonical form to which matrix 4-tuples can be reduced, using display operations [Definition 6.5] and sweeping-similarity operations [Theorem 6.7]. As in the previous section, this was solved in $[\mathbf{L 1}, \mathbf{L 3}]$, although there is a better proof in [KL0]. Therefore, our object in the present section is to focus on establishing consistency with the notation of the present series, and connecting with the proof in [KLO].

Our canonical forms of indecomposable 4-tuples are of two types that we call "deleted cycle" and "block cycle" 4-tuples.

Throughout this section $(\Lambda, \mathfrak{m}, k)$ is strictly split Dedekind-like with normalization $\Gamma$, and all matrices have entries in $k$.

Definition 7.1 (Direct sum of 4-tuples). We define the direct sum

$$
\left(A_{1}, A_{2}, B_{1}, B_{2}\right)=\left(A_{1}^{\prime}, A_{2}^{\prime}, B_{1}^{\prime}, B_{2}^{\prime}\right) \oplus\left(A_{1}^{\prime \prime}, A_{2}^{\prime \prime}, B_{1}^{\prime \prime}, B_{2}^{\prime \prime}\right)
$$

of matrix 4-tuples to be the expected matrix 4-tuple: $A_{1}=\left[\begin{array}{cc}A_{1}^{\prime} & 0 \\ 0 & A_{1}^{\prime \prime}\end{array}\right]$ with length labels those of $A_{1}^{\prime}$ followed by those of $A_{1}^{\prime \prime}$, and the remaining three matrices in this 4-tuple defined similarly.

For example, consider the following matrix 4-tuple, where the numbers $3,6,3, \infty, 5$ are the length labels of the columns of $A_{1}$ as well as the corresponding columns of $B_{1}$, and an analogous comment applies to $A_{2}$ and $B_{2}$. 


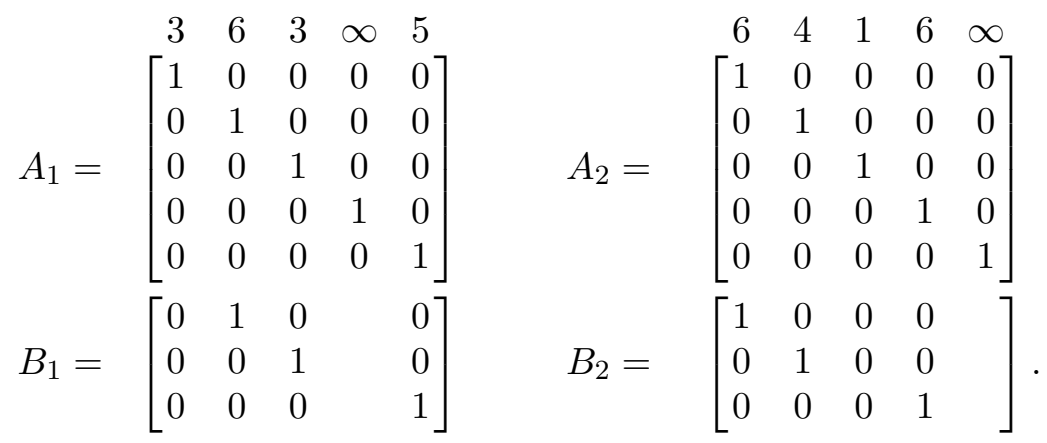

This 4-tuple is the direct sum of the two 4-tuples whose $A$-matrices are identity matrices of appropriate sizes and whose $B$-matrices, including their length labels, are

$$
\begin{aligned}
& \left.\left(B_{1}^{\prime}, B_{2}^{\prime}\right)=\left(\begin{array}{ccc}
3 & 6 & 3 \\
0 & 1 & 0 \\
0 & 0 & 1
\end{array}\right] \quad, \quad\left[\begin{array}{lll}
1 & 0 & 0 \\
0 & 1 & 0
\end{array}\right]\right) \\
& \left(B_{1}^{\prime \prime}, B_{2}^{\prime \prime}\right)=\left(\begin{array}{llll}
\infty & 5 & 6 & \infty \\
{[} & 1
\end{array}\right], \quad\left[\begin{array}{ll}
1 & ]
\end{array}\right) \text {. }
\end{aligned}
$$

Note that $B_{1}^{\prime \prime}$ and $B_{2}^{\prime \prime}$ are $1 \times 1$ matrices, with no actual columns labeled by $\infty$.

Definitions 7.2 (Canonical forms). We call our first canonical form a deleted cycle 4-tuple. It is determined by:

(7.2.1) A pair of label sequences $i_{1}, i_{2}, \ldots, i_{d}$ and $j_{1}, j_{2}, \ldots, j_{d}(d \geq 1)$, where each term is a positive integer or $\infty$, and length labels $\infty$ and 1 cannot occur except possibly as $i_{1}$ or $j_{d}$.

The deleted cycle matrix 4-tuple determined by these sequences is defined as follows. Set $A_{1}=A_{2}=I_{d}$, with the length labels of $A_{1}$ and $A_{2}$ given respectively by the two sequences in (7.2.1). The matrices $B_{1}$ and $B_{2}$ are as displayed in (7.2.2).

$$
\left.\left(B_{1}, B_{2}\right)=\left(\begin{array}{cc}
i_{1} & \cdots \\
Z & I_{d-1}
\end{array}\right],\left[\begin{array}{ll}
I_{d-1} & Z^{\prime}
\end{array}\right]\right)
$$

Here, the dots over the first $I_{d-1}$ indicate that its columns are labeled by $i_{2}, \ldots, i_{d}$ and the dots over the second $I_{d-1}$ indicate that its columns are labeled by $j_{1}, \ldots, j_{d-1}$. Column $Z$ consists of zeros if the label $i_{1}$ is finite, but the column is not present if $i_{1}=\infty$. Similarly, $Z^{\prime}$ is a column of zeros if the label $j_{d}$ is finite, but is not present if $j_{d}=\infty$.

If, for example, $i_{1}$ is finite and $j_{d}$ infinite, then the sizes of $B_{1}$ and $B_{2}$ are $(d-1) \times d$ and $(d-1) \times(d-1)$ respectively, and $Z^{\prime}$ indicates that the $\infty$-labeled column of $A_{2}$ has no corresponding column in $B_{2}$. 
As a second example, the 4-tuple displayed in (7.1.1) is the direct sum of the two deleted cycle 4-tuples whose $B$-matrices are displayed in (7.1.2).

We call our other canonical form a block cycle 4-tuple. It is determined by:

(7.2.3) (i) A pair of label sequences $i_{1}, i_{2}, \ldots, i_{d}$ and $j_{1}, j_{2}, \ldots, j_{d}(d \geq 1)$, where each term is a positive integer not equal to 1 (never $\infty$ !), and such that the sequence of pairs $\left(i_{1}, j_{1}\right), \ldots,\left(i_{d}, j_{d}\right)$ is repetition-free.

(ii) An invertible matrix $L$ (over $k$ ), indecomposable under similarity, called the blocking matrix. The number of rows of $L$ is called the block size of the matrix 4-tuple.

(Recall, from (2.6.1), that a finite sequence $W$ is repetition-free if there is no strictly shorter sequence $W^{\prime}$ such that $W$ consists of repetitions of $W^{\prime}$.)

We display in (7.2.4) the block cycle 4-tuple determined by the data (7.2.3), and then explain it.

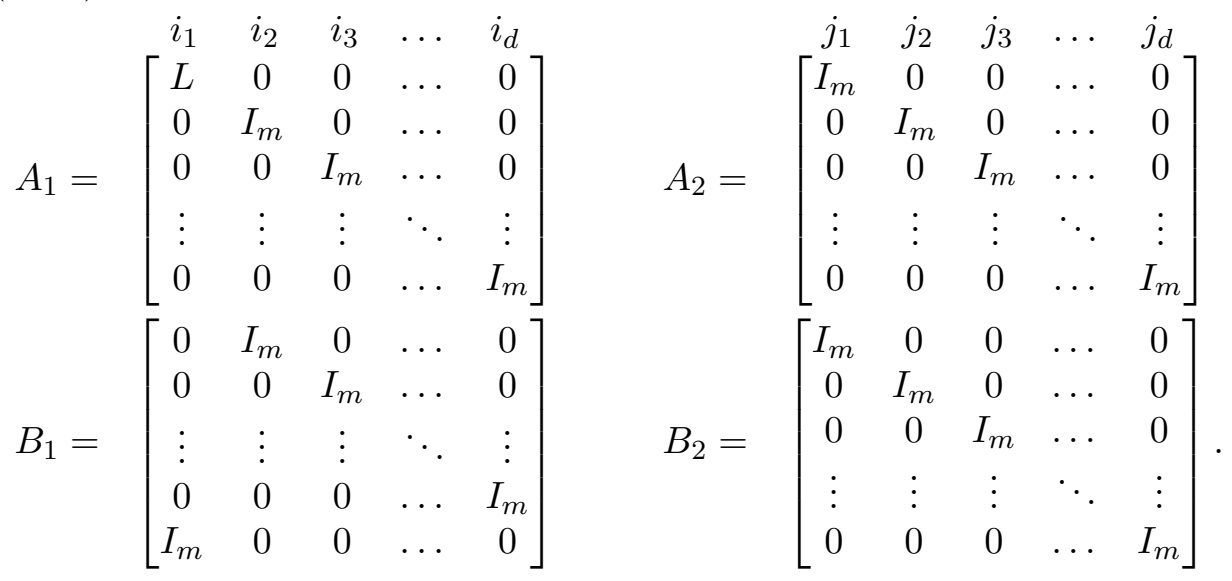

Let the block size be $m$. Thus the size of $L$ is $m \times m$, and each matrix in the 4-tuple has size $d m \times d m$. Matrices $A_{1}$ and $A_{2}$ have the displayed block diagonal form, with all blocks of size $m \times m$, the upper left-hand block of $A_{1}$ equals $L$, and all other diagonal blocks equal $I_{m}$. Each term of each label sequence appears $m$ times as a length label (although we display it only once here), thus labeling all $m$ columns of the block over which it is displayed. The matrix $B_{1}$ has identity blocks on the super-diagonal, one identity block in the lower left-hand corner, and zeros elsewhere. Thus - as suggested by the name "block cycle" $-B_{1}$ is a block permutation matrix whose associated permutation is a cycle. We also have $B_{2}=I_{d m}=A_{2}$. We emphasize that $\infty$ and 1 never occur in the label sequences of a block cycle 4-tuple. 
Recall [Notation 6.9] that two matrix 4-tuples are called isomorphic if one can be transformed into the other by means of display operations and $k-k$ sweeping-similarity operations. Let $I, J$ and $I^{\prime}, J^{\prime}$ be two pairs of finite sequences, each sequence of the same length, say $d$. Recall that $I, J$ and $I^{\prime}, J^{\prime}$ are said to be equal modulo simultaneous cyclic permutations if there is an integer $c$ such that $I^{\prime}=\nu^{c}(I)$ and $J^{\prime}=\nu^{c}(J)$. (See Notation 2.3.) Finally, we call a matrix 4-tuple indecomposable if it is not isomorphic to the direct sum of two matrix 4-tuples of strictly smaller size.

Theorem 7.3. (i) Every deleted cycle 4-tuple is indecomposable. Two deleted cycle 4-tuples are isomorphic if and only if they are equal which holds if and only if they have the same pair of label sequences.

(ii) Every block cycle 4-tuple is indecomposable. Two block cycle 4-tuples are isomorphic if and only if their pairs of label sequences are equal modulo simultaneous cyclic permutations and their blocking matrices are similar.

(iii) A deleted cycle 4-tuple is is never isomorphic to a block cycle 4-tuple.

Since only the similarity class of $L$ is important, $L$ can always be chosen to be the companion matrix of some power $g(X)^{e}$ of some irreducible polynomial $g(X) \neq X$ over $k$. Recall that "display operations" were defined in 6.5 .

Theorem 7.4. Every matrix 4-tuple is isomorphic to a direct sum of deleted cycle and block cycle 4-tuples. Moreover, two direct sums of deleted cycle and block cycle 4-tuples are isomorphic if and only if they contain the same deleted cycle summands and isomorphic block cycle summands, with the same multiplicities but ignoring order of occurrence.

Proof. These two theorems are a special case of [KL0, 1.3-1.7]. So we limit ourselves to a few comments relating the notation here to the notation there.

First note that the sweeping-similarity operations in Theorem 6.7 form a set of sweeping-similarity operations, as described in [KL0, p. 68], with one change: All sweeping in that paper is done to the right. But this is just a matter of notation, only affecting the way in which the problem is displayed. In our situation, no actual row-sweeping occurs; that is, all rows of $A_{1}$ belong to a single row-block (as defined in [KL0]), and the same is true of the other three matrices.

Second, note that the present paper uses a slightly more generous definition of "display operation" than was given in [KL0, (2.5.2)]. In that earlier paper, unlike the present paper, display operations were not allowed to change the order of two columns with the same label. However, a pair of columns in $A_{i}$ and the corresponding pair of columns in $B_{i}$ may be simultaneously permuted by means of a sweeping-similarity operation. Therefore the more generous definition of "display operations" does not change what 
can be done by combining display operations with sweeping-similarity operations.

Having made these observations, one then applies the aforementioned theorems from [KL0].

\section{Solution of matrix problem, unsplit case.}

Throughout this section we assume that $(\Lambda, \mathfrak{m}, k)$ is unsplit Dedekind-like with normalization $(\Gamma, \mathfrak{m}, F)$. In this section we actually use the assumption (not invoked earlier in the paper) that the 2-dimensional field extension $F$ of $k$ is separable. [See (1.1.3).] Our objective is to find a canonical form for indecomposable matrix pairs $p=(A, B)$ (entries in $F$ ). Since this is the most complicated section in the paper, we begin with a brief summary of what will be done.

Since $F$ is Galois of dimension 2 over $k$, every matrix $C$ over $F$ has a natural conjugate $\bar{C}$ (with the same length labels as $C$ if $C$ is part of a matrix pair or 4-tuple). Given a matrix pair $p$ over the field $F$, our plan is to associate with $p=(A, B)$ the matrix 4-tuple $f(p)=(A, \bar{A}, B, \bar{B})$. Our solution of the problem then falls into four parts.

(i) Show that $p \cong p^{\prime}$ if and only if $f(p) \cong f\left(p^{\prime}\right)$ (Theorem 8.3). This reduces isomorphism in the unsplit case to isomorphism in the strictly split case, whose canonical forms were summarized in the previous section. But our 4-tuples now have entries in $F$ instead of $k$.

(ii) For each self-conjugate indecomposable 4-tuple $g$ we describe a pair $p$ such that $f(p) \cong g$, and for each non-self-conjugate indecomposable 4tuple $g$ we describe a pair $p$ such that $f(p) \cong g \oplus \bar{g}$. We obtain seven types of canonical forms $p$ in this way, and it is easy to see that each is an indecomposable pair.

Our proof that every self-conjugate indecomposable 4-tuple has the form $f(p)$, for some matrix pair $p$, uses a "Hilbert Theorem 90" for matrices, due to Ballantine [Lemma 8.11].

(iii) We show that the list of canonical forms in (ii) is complete. The critical theorem is that a 4 -tuple $g$ is a "package" — that is, $g \cong f(p)$ for some pair $p$ - if and only if $g \cong \bar{g}$. The proof of this seems to require the list of seven canonical forms listed in (ii).

An easy consequence of all of this is that the Krull-Schmidt Theorem holds for matrix pairs.

(iv) The final main theorem in this section describes the precise extent to which our canonical forms are uniquely determined by their isomorphism class.

The results of the present section are purely matrix-theoretic results involving any pair of fields $k$ and $F$, with $F$ separable of degree 2 over $k$. 
In other words, $\Lambda$ and $\Gamma$ are really irrelevant. In fact we use this observation at one critical point in the proof of part (i). But by keeping $\Lambda$ and $\Gamma$ in the background (rather than omitting them) we avoid the necessity of introducing new terminology.

Notation 8.1. Since $F$ is separable of dimension 2 over $k$, there is precisely one nonidentity automorphism of $F$ that equals the identity on $k$. We denote the image of $\alpha \in F$, under this automorphism, by $\bar{\alpha}$, the conjugate of $\alpha$.

Let $p=(A, B)$ be a matrix pair associated with a matrix setup $\mathcal{X}$ (unsplit case) [Notation 5.7], and recall that every column of $A$ and $B$ has an attached length label, a positive integer or $\infty$. Let $f(p)=(A, \bar{A}, B, \bar{B})$, the matrix 4-tuple in which "bar" indicates that we take the conjugate of every entry of the matrix. The length labels of $f(p)$ are obtained by keeping the original length labels of $A$ and $B$, and giving $\bar{A}$ and $\bar{B}$ the same length labels as $A$ and $B$, respectively. We call $f(p)$ the matrix 4-tuple associated with $p$. It is straightforward to verify that the length labels of $f(p)$ are a legitimate set of length labels for some matrix setup in the strictly split case [Notation 6.3], and $f(p)$ is a legitimate matrix 4-tuple associated with that setup [Notation 6.4].

Recall [Notation 5.14] that, for matrix pairs $p$ and $p^{\prime}$, we write $p \cong p^{\prime}$ if $p^{\prime}$ can be obtained from $p$ by display operations and $k-F$ sweeping-similarity operations [Theorem 5.11].

Let $g=\left(C_{1}, C_{2}, D_{1}, D_{2}\right)$ and $g^{\prime}=\left(C_{1}^{\prime}, C_{2}^{\prime}, D_{1}^{\prime}, D_{2}^{\prime}\right)$ be matrix 4-tuples with entries in $F$ (associated with suitable matrix setups for a strictly split Dedekind-like ring with residue field $F$ ). We write $g \cong g^{\prime}$ if $g^{\prime}$ can be obtained from $g$ by display operations and $F-F$ sweeping-similarity operations, the the analog over $F$ of the $k$ - $k$ sweeping-similarity operations of Theorem 6.7.

The conjugate of the matrix 4-tuple $g=\left(C_{1}, C_{2}, D_{1}, D_{2}\right)$ is the 4-tuple $\bar{g}=\left(\bar{C}_{2}, \bar{C}_{1}, \bar{D}_{2}, \bar{D}_{1}\right)$ obtained by interchanging $C_{1}$ with $C_{2}$, together with their length labels, doing the same with $D_{1}$ and $D_{2}$, and then taking the conjugates of all four matrices. We call the matrix 4-tuple $g$ self-conjugate if $g \cong \bar{g}$. We call the matrix 4-tuple $g$ a package if $g \cong f(p)$ for some matrix pair $p$. We call a package $g$ indecomposable if it is not the direct sum of two packages.

From the definitions, it is evident that every package is self-conjugate. The converse of this is also true, as we show in Theorem 8.15.

We call $F \otimes_{k} F$ a left $F$-algebra when we use the scalar product $\gamma \cdot(\alpha \otimes \beta)=$ $\gamma \alpha \otimes \beta$. The notation $F[[X]]$ denotes the ring of formal power series in an indeterminate $X$ over $F$.

Lemma 8.2. (i) $F \otimes_{k} F \cong F \oplus F$ as left $F$-algebras via the map defined by $\tau(\alpha \otimes \beta)=(\alpha \beta, \alpha \bar{\beta})$. 
(ii) $F \otimes_{k} F[[X]] \cong F[[X]] \oplus F[[X]]$ as left $F$-algebras via the extension $\tau^{\prime}(\alpha \otimes f)=(\alpha f, \alpha \bar{f})$ of the map in (i), where $\bar{f}$ is the result of replacing every coefficient of $f$ by its conjugate over $k$.

Proof. (i) This well-known fact is easily proved by first choosing any $\varepsilon \in$ $F-k$ and noting that $F=k[\varepsilon]$ since $F$ has dimension 2 over $k$. Since, in addition, $F$ is separable over $k$, the minimal polynomial of $\varepsilon$ over $k$ is $m(X)=(X-\varepsilon)(X-\bar{\varepsilon})$ and has distinct linear factors. Therefore $F \otimes_{k} F \cong$ $F \otimes(k[X] /(m(X))) \cong F[X] /(m(X)) \cong F[X] /(X-\varepsilon) \oplus F[X] /(X-\bar{\varepsilon})$, and the result follows easily.

(ii) Note that $\tau^{\prime}$ is obviously a homomorphism of left $F$-algebras; we need to show that $\tau^{\prime}$ is a bijection. Since $F \otimes_{k} F$ is an $F$-F-bimodule, we can form the following tensor product and map:

$$
\tau \otimes 1:\left(F \otimes_{k} F\right) \otimes_{F} F[[X]] \rightarrow(F \oplus F) \otimes_{F} F[[X]] .
$$

Then we can use the identification $F \otimes_{F} F[[X]]=F[[X]]$ to identify $\tau^{\prime}$ with $\tau \otimes 1$. Since $\tau$ is an isomorphism, by (i), and $\tau^{\prime}$ is the tensor product of two isomorphisms, $\tau^{\prime}$ is itself an isomorphism.

Theorem 8.3. Let $p$ and $p^{\prime}$ be matrix pairs. Then $p \cong p^{\prime}$ if and only if $f(p) \cong f\left(p^{\prime}\right)$.

Proof. This proof is an adaptation a familiar "extension of scalars" argument. The main difficulty to be surmounted is that $\Lambda$ need not be an algebra over a field.

First note that the matrix assertions in the theorem ultimately depend only on the pair of fields $k$ and $F$. Hence we can choose $\Lambda$ to be any convenient unsplit Dedekind-like ring such that the bottom row of its pullback diagram (1.1.1) is the inclusion $k \subset F$. Let $\Gamma=F[[X]]$ and $\Lambda=k+x F[[x]]$. Then $\Lambda$ is an unsplit Dedekind-like ring with maximal ideal $\mathfrak{m}=x F[[X]]$ and residue field $k$, and the normalization of $\Lambda$ is $\Gamma=(F[[X]], \mathfrak{m}, F)$. This is displayed in the first pullback square in (8.3.1) below, where $\operatorname{ker} \rho=\mathfrak{m}$.
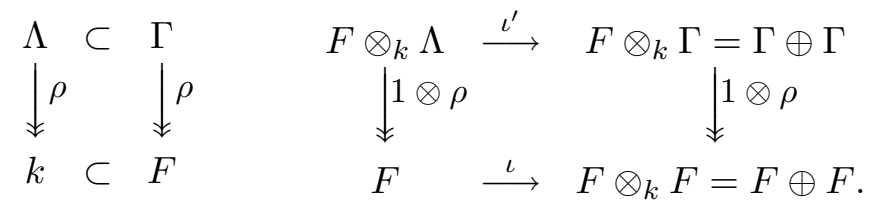

To obtain the second square in (8.3.1), tensor the first square with $F$ over $k$, as shown, making the identifications that we proceed to explain. Identify the $F$ in the lower left corner with $F \otimes_{k} k$ in the natural way, and let the two vertical maps be as shown. The two horizontal maps are simply $1 \otimes i$ ( $i$ the relevant inclusion map). Then the diagram commutates.

The equalities in the upper right corner and lower right corner refer to the isomorphisms $\tau^{\prime}$ and $\tau$, respectively, of Lemma 8.2, both of which we regard as identification. Then the lower horizontal map $\iota$ is the composition of two 
identifications and $1 \otimes i$, and as $\operatorname{such} \iota(\alpha)=(1 \otimes i)(\alpha \otimes 1)=\alpha \otimes 1=(\alpha, \alpha)$ for each $\alpha \in F$; that is, $\iota$ is the diagonal inclusion of $F$ into $F \oplus F$.

This square is a pullback diagram because tensoring a pullback diagram by a flat module again yields a pullback diagram (use the idea in e.g. $[\mathbf{K}$, Proposition 2.10] or [L3, Lemma 6.1]). This shows that $F \otimes_{k} \Lambda$ is the strictly split Dedekind-like ring whose pullback diagram is the second square displayed in (8.3.1).

Now we have a pair $\Lambda, \Gamma$ to use in a standard extension of scalars argument. Since the argument involves a lot of details, we describe only the main steps. Let $M, N$ be finitely generated $\Lambda$-modules. Then we claim:

$$
M \cong N \text { (as } \Lambda \text {-modules }) \Longleftrightarrow F \otimes_{k} M \cong F \otimes_{k} N \text { (as } F \otimes_{k} \Lambda \text {-modules). }
$$

For the proof of the nontrivial $(\Leftarrow)$ assertion, first note that, since $F$ is a 2dimensional $k$-vector space, the second isomorphism implies that $M \oplus M \cong$ $N \oplus N$ as $\Lambda$-modules. Then use the Krull-Schmidt Theorem for strictly split Dedekind-like rings [Lemma 1.3].

Let $\Lambda^{\prime}=F \otimes_{k} \Lambda$ and $\Gamma^{\prime}=F \otimes_{k} \Gamma=\Gamma \oplus \Gamma$. When it is necessary to distinguish between the two direct summands of $\Gamma^{\prime}$, we write $\Gamma^{\prime}=\Gamma_{1} \oplus \Gamma_{2}$. (Note that $\Gamma_{1} \cong \Gamma_{2}$ as $F$-algebras and $\Gamma$-modules, but not as $\Gamma^{\prime}$-modules, being annihilated by $(0, \Gamma)$ and $(\Gamma, 0)$, respectively.) The length labels of our given matrix pair $p$ determine the $\Gamma$-module $X$ in some matrix setup $\mathcal{X}$ for $\Lambda$, and the length labels of $f(p)$ determine the $\Gamma^{\prime}$-module $X^{\prime}=X_{1} \oplus X_{2}$ in some matrix setup $\mathcal{X}^{\prime}$ for $\Lambda^{\prime}$. In fact, one verifies with the help of Lemma 8.2(ii), that for every indecomposable summand $Y=\Gamma / \mathfrak{m}^{i}(1 \leq i \leq \infty)$ in the definition of $X$, we have $F \otimes_{k} Y=Y_{1} \oplus Y_{2}$, where $Y \cong Y_{1} \cong Y_{2}$ as $\Gamma$ modules, (but not as $\Gamma^{\prime}$-modules).

It now suffices to show that

$$
F \otimes_{k} M(A, B) \cong M(A, \bar{A}, B, \bar{B}) \quad\left(\text { as }\left(F \otimes_{k} \Lambda\right) \text {-modules }\right)
$$

where $p=(A, B)$ and hence $f(p)=(A, \bar{A}, B, \bar{B})$. For it then follows from (8.3.2) that $M(p) \cong M\left(p^{\prime}\right)$ if and only if $M(f(p)) \cong M\left(f\left(p^{\prime}\right)\right)$, after which our two theorems on modules versus matrices 5.15 and 6.10 (unsplit and split cases, respectively) imply that $p \cong p^{\prime}$ if and only if $f(p) \cong f\left(p^{\prime}\right)$.

The proof of (8.3.3) consists of reviewing the definitions of the maps in the definitions of $M(A, B)$ and $M(A, \bar{A}, B, \bar{B})$ and noting that, for every map $\theta$ that arises in the definition of $M(A, B)$, the map $1 \otimes \theta$ is the direct sum of two corresponding maps in the definition of $M(A, \bar{A}, B, \bar{B})$, and for every entry $\beta$ of $A$ (respectively $B$ ), the tensor product $1 \otimes \beta$ corresponds to a pair of entries $(\beta, \bar{\beta})$ of the matrices $A$ and $\bar{A}$ (respectively $B$ and $\bar{B}$ ), under the map $\tau$ of Lemma (8.2)(i).

Lemma 8.4. Given $\varepsilon \in F-k$, let $Y$ be an invertible $m \times m$ matrix over the field $F$, and consider the conjugate pair of $2 m \times 2 m$ matrices $T$ and $\bar{T}$ 
below.

$$
T=\left[\begin{array}{cc}
Y & I_{m} \\
\varepsilon Y & \bar{\varepsilon} I_{m}
\end{array}\right], \quad \bar{T}=\left[\begin{array}{cc}
\bar{Y} & I_{m} \\
\bar{\varepsilon} \bar{Y} & \varepsilon I_{m}
\end{array}\right] .
$$

Then there is an invertible matrix $P$ over $F$ such that $P T$ and $P \bar{T}$ equal the following two matrices, respectively.

$$
\left[\begin{array}{cc}
Y & 0 \\
0 & I_{m}
\end{array}\right], \quad\left[\begin{array}{cc}
0 & I_{m} \\
\bar{Y} & 0
\end{array}\right] .
$$

(We refer to the matrix $T$ as a "basic tile.")

Proof. We prove the lemma by performing simultaneous row operations on the matrices in (8.4.1). Subtracting $\varepsilon$ times the first $m$ rows from the last $m$ rows in both matrices yields:

$$
\left[\begin{array}{cc}
Y & I_{m} \\
0 & \delta I_{m}
\end{array}\right], \quad\left[\begin{array}{cc}
\bar{Y} & I_{m} \\
\delta \bar{Y} & 0
\end{array}\right]
$$

where $\delta=\bar{\varepsilon}-\varepsilon \neq 0$, since $F$ is separable over $k$. Dividing the last $m$ rows by $\delta$ in both matrices in (8.4.3) and then subtracting the last $m$ rows from the first $m$ rows yields the desired matrices (8.4.2).

The following simple lemma helps to motivate our systematic enumeration of canonical forms for matrix pairs (but does not show that the resulting list is complete).

Lemma 8.5. Let $p$ be a matrix pair such that $f(p)$ is either an indecomposable self-conjugate 4-tuple, or else $f(p) \cong g \oplus \bar{g}$ where $g$ is indecomposable and non-self-conjugate. Then $p$ is indecomposable (equivalently, $f(p)$ is an indecomposable package).

Proof. Only the case $f(p) \cong g \oplus \bar{g}$ is nontrivial. So suppose that $p \cong q \oplus r$, and hence $f(p) \cong f(q) \oplus f(r)$. Since $g$ is indecomposable, so is $\bar{g}$. Since the Krull-Schmidt Theorem holds for matrix 4-tuples [Theorem 7.4], we must therefore have, say, $f(q) \cong g$. But, since $f(q)$ is a package, it is obviously self-conjugate. Therefore $g \cong \bar{g}$, a contradiction.

The following notation will be fundamental throughout this section.

Notation 8.6 (Sequence manipulation). Given the finite sequence $I=\left\{x_{1}\right.$, $\left.\ldots, x_{d}\right\}$, we let $\mu$ be the mirror image permutation that reverses the order of $I$; that is, $\mu(I)=\left\{x_{d}, x_{d-1}, \ldots, x_{1}\right\}$. We let $\nu$ be the unit forward rotation, that is, the cyclic permutation defined by $\nu(I)=\left\{x_{2}, x_{3}, \ldots, x_{d}, x_{1}\right\}$. A cycle is any cyclic permutation of the form $\nu^{t}$, where $t$ is an integer. Thus, $\nu^{t}(I)=\left\{x_{t+1}, x_{t+2}, \ldots, x_{t-1}, x_{t}\right\}$ (reading subscripts modulo $d$ ). We frequently use the following, easily verified relation.

$$
\nu^{t} \mu=\mu \nu^{-t} \text {. }
$$


For finite sequences $I$ and $J$ we use the notation $\{I, J\}$ for the concatenation of $I$ and $J$, that is, the sequence consisting of the terms of $I$ followed by the terms of $J$.

Definition 8.7 (Pairs in canonical form arising from deleted cycle 4-tuples). For each pair $p=(A, B)$ that we describe here, we shall show that $f(p)$ is isomorphic to the direct sum of one or two deleted cycle 4-tuples [Theorem 8.9]. The name that we use for each individual form is chosen to coincide with the name of the corresponding type of module, described in $\S 2$.

All blank spaces in our matrix displays - except ellipsis dots - represent zeros; and $\varepsilon$ denotes any element of $F-k$ (hence $F=k[\varepsilon]$ ).

The data from which each matrix pair is constructed is a pair of label sequences

$$
I=\left\{i_{1}, i_{2}, \ldots, i_{d}\right\} \quad \text { and } \quad J=\left\{j_{1}, j_{2}, \ldots,\left[j_{d}\right]\right\}
$$

in which each term is a positive integer or $\infty$. The term $j_{d}$ is in brackets because, in some types, it is not present.

Remark. Note that we use a pair of label sequences to define the deleted cycle matrix pairs below, even though a matrix pair $(A, B)$ needs only a single label sequence for labeling the columns of $A$ (and hence the corresponding columns of $B$ ). As we shall see below, the two label sequences $I$ and $J$ are woven together to form a single label sequence for the matrix pairs to be defined. The reason for splitting into two sequences $I$ and $J$ in this definition is that two label sequences are needed by matrix 4-tuples. (See Theorem 8.9 below.)

Nonreduced pair. Label $j_{d}$ occurs. The label sequences must satisfy:

(8.7.2) (i) Only labels $i_{1}$ and $j_{d}$ can equal $\infty$ or 1 .

(ii) The pair of label sequences $I$ and $J$ must be unsymmetrical, in the sense that $J \neq \mu(I)$ (the mirror image of $I$ ).

The form of these matrices is displayed in (8.7.3), below, in which the row of length labels at the top, comes from the pair of label sequences. Each column of $Z$ 's is a column of zeros except if the length label is $\infty$, in which case that column is not present in $B$ (because no column of $B$ is allowed to have an infinite length label). Note that $A$ is a direct sum of $2 \times 2$ basic tiles (8.4.1), while $B$ is a modified such direct sum. The basic tiles that appear in $A$ always overlap the tiles that appear in $B$, as shown.

We shall show, for nonreduced pairs $p$, that $f(p)$ is isomorphic to the direct sum of two non-self-conjugate deleted cycle 4-tuples [Theorem 8.9]. The reason for nonsymmetry condition (8.7.2)(ii) is that, when it is violated, $f(p)$ becomes isomorphic to the direct sum of two self-conjugate 4-tuples, and $p$ itself is isomorphic to the direct sum of two isomorphic indecomposable pairs [Proposition 8.19]. 


$$
\begin{aligned}
& \begin{array}{llllllllll}
i_{1} & j_{1} & i_{2} & j_{2} & i_{3} & \ldots & j_{d-1} & i_{d} & j_{d}
\end{array} \\
& A=\left[\begin{array}{cccccccc}
1 & 1 & & & \ldots & & \\
\varepsilon & \bar{\varepsilon} & & & \cdots & & \\
& & 1 & 1 & \ldots & & \\
& & \varepsilon & \bar{\varepsilon} & \cdots & & \\
& & & & \ddots & & \\
& & & & \cdots & 1 & 1 \\
& & & & \cdots & \varepsilon & \bar{\varepsilon}
\end{array}\right] \\
& B=\left[\begin{array}{ccccccccc}
Z & 1 & 1 & & & \ldots & & & Z \\
Z & \varepsilon & \bar{\varepsilon} & & & \cdots & & & Z \\
Z & & & 1 & 1 & \cdots & & & Z \\
Z & & & \varepsilon & \bar{\varepsilon} & \cdots & & & Z \\
\vdots & & & & & \ddots & & & \vdots \\
Z & & & & & \ldots & 1 & 1 & Z \\
Z & & & & & \cdots & \varepsilon & \bar{\varepsilon} & Z
\end{array}\right]
\end{aligned}
$$

Bottom-reduced pair. Label $j_{d}$ occurs. We require the label sequences to satisfy:

(8.7.4) Only label $i_{1}$ can equal $\infty$ or 1.

(There is no symmetry restriction.) The matrices are the same as in the nonreduced case, except that $B$ has the following additional row at its bottom.

$$
\begin{array}{rlllllll}
i_{1} & j_{1} & i_{2} & j_{2} & i_{3} & \ldots & i_{d} & j_{d} \\
(B:) & {[}
\end{array}
$$

where $Z$ denotes zero except if $i_{1}=\infty$, in which case that column of $B$ is not present. As usual, all entries in this row that are not explicitly shown equal 0 . In the extreme case $d=1$, the new bottom row consists only of $Z$ (if it is present) and 1.

Top-reduced pair. In this case, label $j_{d}$ is not present. We require the label sequences to satisfy (8.7.4), and there is no symmetry restriction. The matrix pair takes the form given in (8.7.6), below, in which the previously discussed conventions apply. In the extreme case $d=1, A$ is the one-byone identity matrix (i.e., no basic tiles occur) and $B$ is the one-by-one zero matrix, except if $i_{1}=\infty$, in which case the matrix $B$ does not occur. 


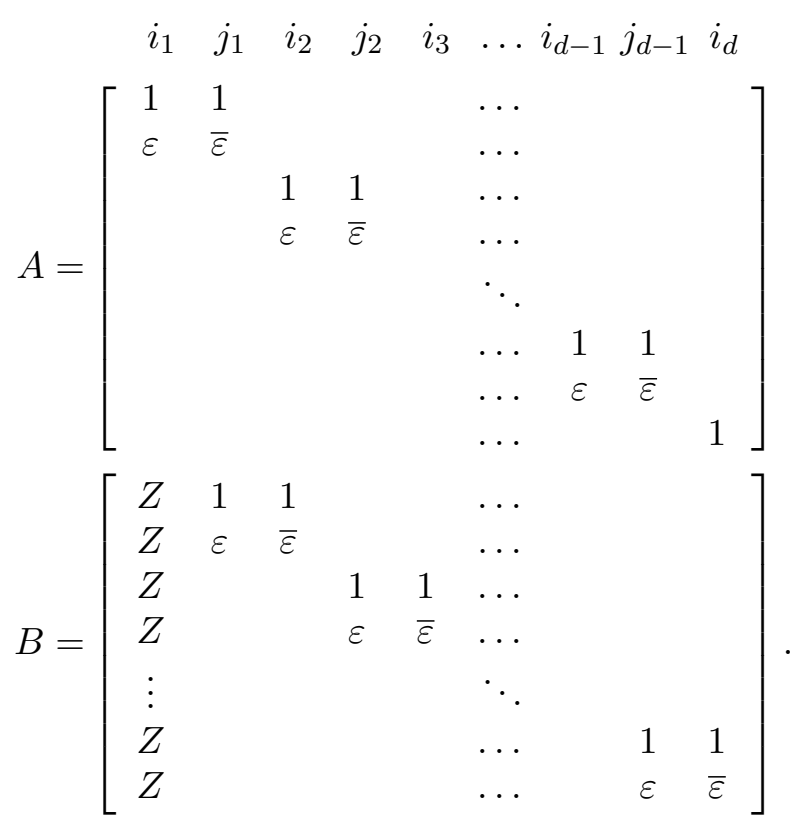

We shall show [Theorem 8.9] that, if $p$ is either of the reduced pairs, then $f(p)$ is a self-conjugate deleted cycle 4-tuple. First, we need to determine which deleted cycle 4-tuple is isomorphic to the conjugate of a given deleted cycle 4-tuple.

Lemma 8.8. Let $g$ and $h$ be the deleted cycle 4-tuples whose label sequences are:

$$
\begin{aligned}
g: H & =\left\{i_{1}, i_{2}, \ldots, i_{e}\right\} \quad \text { and } \quad K=\left\{j_{1}, j_{2}, \ldots, j_{e}\right\} \\
h: \mu(K) & =\left\{j_{e}, j_{e-1}, \ldots, j_{1}\right\} \quad \text { and } \mu(H)=\left\{i_{e}, i_{e-1}, \ldots, i_{1}\right\} .
\end{aligned}
$$

Then $\bar{g} \cong h$. In particular, the deleted cycle 4-tuple $g$ is self-conjugate if and only if $K=\mu(H)$.

Proof. We have $g=\left(I_{e}, I_{e},\left[Z I_{e-1}\right],\left[I_{e-1} Z\right]\right)$ with length labels as displayed. Therefore we have $\bar{g}=\left(I_{e}, I_{e},\left[I_{e-1} Z\right],\left[\begin{array}{ll}Z & I_{e-1}\end{array}\right]\right)$, and the label sequences of $\bar{g}$ are the pair $K$ and $H$. The 4 -tuple $\bar{g}$ is not a deleted cycle 4 -tuple. We transform $\bar{g}$ to the deleted cycle 4-tuple $h$.

Let $h^{\prime}$ be the 4 -tuple obtained from $\bar{g}$ by the display operation that replaces the pair of label sequences by $\mu(K)$ and $\mu(H)$, respectively. This reverses the order of columns of each $A_{i}$, together with their corresponding columns in the $B$-matrices. Then $h^{\prime} \cong \bar{g}$ and the label sequences are now as desired. The first and second matrices of $h^{\prime}$ are now the matrix $R_{e}$ obtained by reversing the columns of $I_{e}$. The third and fourth matrices are now $\left[Z R_{e-1}\right]$ and $\left[R_{e-1} Z\right]$. Therefore, left multiplying the first and second 
matrices of $h^{\prime}$ by $R_{e}^{-1}$ changes these two matrices to $I_{e}$ and left-multiplying

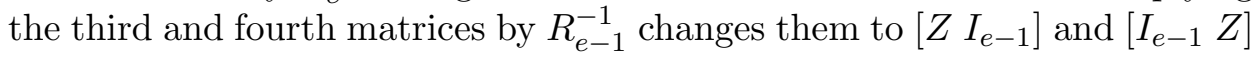
respectively. In other words, we have now changed $h^{\prime}$ to $h$, as desired.

The last statement now follows from Theorem 7.3.

Theorem 8.9. Let $p=(A, B)$ be any of the types of matrix pairs listed below, constructed from the pair of label sequences

$$
I=\left\{i_{1}, \ldots, i_{d-1}, i_{d}\right\} \quad \text { and } \quad J=\left\{j_{1}, \ldots, j_{d-1},\left[j_{d}\right]\right\}
$$

Then $f(p)$ is as described below, where $\mu$ is the mirror image permutation as in Notation 8.6.

(i) $p$ is nonreduced. Then $f(p)$ is isomorphic to $g \oplus \bar{g}$ where $g$ is the nonself-conjugate deleted cycle 4-tuple whose pair of label sequences is I and $J$.

(ii) $p$ is bottom-reduced. Then $f(p)$ is isomorphic to the deleted cycle 4tuple $g \cong \bar{g}$ whose pair of label sequences is $\{I, \mu(J)\}$ and $\{J, \mu(I)\}$.

(iii) $p$ is top-reduced. Then $f(p)$ is isomorphic to the deleted cycle 4-tuple $g \cong \bar{g}$ whose pair of label sequences is $\{I, \mu(J)\}$ and $\{J, \mu(I)\}$. (Recall that, in this case, the label $j_{d}$ does not occur.)

In all cases, $p$ is an indecomposable matrix pair (equivalently, $f(p)$ is an indecomposable package). Conversely, if $g$ is a non-self-conjugate deleted cycle 4-tuple, then there is some nonreduced matrix pair $p$ with $f(p) \cong g \oplus \bar{g}$; and if $g$ is a self-conjugate deleted cycle 4-tuple, then there is some bottom reduced or top-reduced matrix pair $p$ with $f(p) \cong g$.

Proof. (ii) Let $g$ and $p$ be as stated. Our plan is to use display operations and $F-F$ sweeping-similarity operations to transform $f(p)$ to $g$ (see Notation 6.9 and Theorem 6.10).

Choose a pair of rows of $A$ and $\bar{A}$ whose nonzero columns consist of a basic tile and its conjugate, as displayed in (8.4.1). The assertion of Lemma 8.4 is that we can transform this pair of tiles to the form displayed in (8.4.2), by simultaneously left-multiplying these two rows of $A$ and $\bar{A}$ by an invertible matrix $P$ over $k$. Since this can be accomplished by left-multiplying $A$ and $\bar{A}$ themselves by an invertible matrix $P^{\prime}$, it is a sweeping-similarity operation. For example, if the two rows that we are altering are rows 1 and 2, we can use the block diagonal matrix $P^{\prime}=\operatorname{diag}(P, I, I, \ldots, I)$. Do this for every pair of basic tiles in $f(p)$, calling the result $h$. 
The form of $h$, when $d=3$ for example, is displayed in (8.9.2) below, with our usual convention that nondisplayed entries equal zero.

$$
\begin{array}{rlrllll}
i_{1} & j_{1} & i_{2} & j_{2} & i_{3} & j_{3} \\
A_{1}=\left[\begin{array}{llllll}
1 & 0 & & & & \\
0 & 1 & & & & \\
& & 1 & 0 & & \\
& & 0 & 1 & & \\
& & & & 1 & 0 \\
& & & & 0 & 1
\end{array}\right] & A_{2}=\left[\begin{array}{llllll}
i_{1} & j_{1} & i_{2} & j_{2} & i_{3} & j_{3} \\
0 & 1 & & & \\
1 & 0 & & & \\
& & 0 & 1 & \\
& & 1 & 0 & & \\
& & & & 0 & 1 \\
& & & & 1 & 0
\end{array}\right] \\
B_{1}=\left[\begin{array}{lllllll}
Z & 1 & 0 & & & \\
Z & 0 & 1 & & & \\
Z & & & 1 & 0 & \\
Z & & & 0 & 1 & \\
Z & & & & 1
\end{array}\right] & B_{2}=\left[\begin{array}{llllll}
Z & 0 & 1 & & & \\
Z & 1 & 0 & & & \\
Z & & & 0 & 1 & \\
Z & & & 1 & 0 & \\
Z & & & & 1
\end{array}\right] .
\end{array}
$$

Note that the columns of $A_{1}$ also occur in $A_{2}$, but in different locations. More precisely:

(8.9.3) For every index $\kappa$, the $i_{\kappa}$-labeled column of $A_{1}$ equals the $j_{\kappa}$-labeled column of $A_{2}$, and the $j_{\kappa}$-labeled colum of $A_{1}$ equals the $i_{\kappa}$-labeled copy of $A_{2}$.

Note that statement (8.9.3) remains true after arbitrary display operations. Let $h^{\prime}$ be the result of doing the display operation that arranges the label sequences as follows:

$$
\{I, \mu(J)\} \quad \text { and } \quad\{J, \mu(I)\} .
$$

Then (8.9.3) implies that the $A_{1}$ and $A_{2}$-matrices of $h^{\prime}$ are equal (ignoring length labels). Call this matrix $C$. It is invertible, in fact, a permutation matrix. Left-multiplying $A_{1}$ and $A_{2}$ by $C^{-1}$ changes $h^{\prime}$ to the form $h^{\prime \prime}=$ $\left(I, I, B_{1}^{\prime \prime}, B_{2}^{\prime \prime}\right)$.

Now consider the $B$-matrices. The $B$-matrices of $h^{\prime \prime}$ are the same as those in (8.9.2) except for having their columns permuted by a display operation. We can therefore find the form of any column of the $B$-matrices of $h^{\prime \prime}$ by finding the column with the same length label in the corresponding matrix in (8.9.2). Moreover, we can simultaneously left-multiply $B_{1}^{\prime \prime}$ and $B_{2}^{\prime \prime}$ by any permutation matrix. In other words, we can arbitrarily permute the order of occurrence of the rows of $\left(B_{1}^{\prime \prime}, B_{2}^{\prime \prime}\right)$. Permute the rows of these two matrices

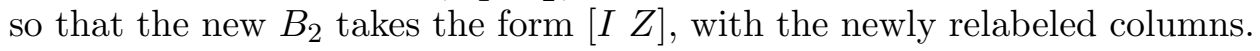
In our example $B_{2}$ is as shown below, and the length labels of $B_{1}$ are also shown in their new order. (The actual arrangement of the entries of $B_{1}$ is 
yet to be determined, so we leave those entries unspecified.)

$$
B_{1}=\left[\begin{array}{cccccc}
i_{1} & i_{2} & i_{3} & j_{3} & j_{2} & j_{1} \\
* & * & * & * & * & * \\
* & * & * & * & * & * \\
* & * & * & * & * & * \\
* & * & * & * & * & * \\
* & * & * & * & * & *
\end{array}\right] \quad B_{2}=\left[\begin{array}{llllll}
1 & & & & & Z \\
& 1 & & & & Z \\
& & 1 & & & Z \\
& & & 1 & & Z \\
& & & & 1 & Z
\end{array}\right] .
$$

This can be done because, if we ignore the Z-column of $B_{2}$ in (8.9.2), $B_{2}$ becomes a permutation matrix.

It now suffices to show that the new $B_{1}$, with its newly rearranged labels and rearranged rows, equals $[Z I]$. For this purpose, return to (8.9.2). The $j_{1^{-}}, j_{2^{-}}$and $j_{3^{-}}$-labeled columns of $B_{2}$ have their nonzero entries in the same rows as the $i_{2^{-}}, i_{3^{-}}$, and $j_{3}$-labeled columns, respectively, of $B_{1}$. Therefore the same is true in the new $\left(B_{1}, B_{2}\right)$. In other words, the first, second, and third rows of the new $B_{1}$ have their nonzero entries in colums $2,3,4$ respectively, as desired. The remaining columns are treated similarly. Also, the $\left(i_{1}\right.$-labeled $)$ $Z$-column occurs in the first column of the new $B_{1}$, as desired.

(iii) This is essentially the same as the proof of (ii), so we omit the details.

(i) This is similar to the proof of (ii). We sketch enough of the proof to show why we get a direct sum of two 4-tuples here, and to establish the following stronger statement that we need later.

(8.9.6) We have $f(p) \cong g \oplus \bar{g}$, even without the nonsymmetry restriction (8.7.2)(ii) in the definition of a nonreduced pair.

Note that the only differences between a nonreduced pair and a bottom reduced pair are the presence of an extra row at the bottom of the latter, and the fact that nonreduced pairs are allowed to have the last column length-labeled by $\infty$.

Begin by reducing all pairs of basic tiles in $f(p)$, as in the proof of (ii), calling the result $h$ as before. To visualize the result in the case $d=3$, delete the bottom row of the $B$-matrices in (8.9.2), and change the entries the last column of $B_{1}$ and $B_{2}$ to $Z^{\prime}$. Continue the reduction until $A_{1}=I=A_{2}$ (ignoring length labels) and - in the case $d=3$ - we reach the situation displayed in (8.9.5), the only difference being that the $j_{3}$-labeled column of $B_{2}$ is now the $Z^{\prime}$-column. (Note that this column is now in the middle of the matrix!) Similarly the $i_{1}$ - and $j_{3}$-labeled columns of $B_{1}$ now have entries $Z$ and $Z^{\prime}$ respectively. The remaining entries of $B_{1}$ are the same as in the bottom-reduced case, and this completes our proof-sketch of this case.

Supplementary statements. Indecomposability is a special case of Lemma 8.5. Completeness of our list follows easily from Lemma 8.8 and the definition of deleted cycle 4-tuples. 
The next step is to do for block cycles what we just did for deleted cycles.

Definition 8.10 (Pairs in canonical form arising from block cycle 4-tuples). The pairs arising in these definitions are constructed from a blocking matrix $U$ - always invertible, of size that we call the block size and usually write $m \times m-$ and a pair of label sequences:

$$
I=\left\{i_{1}, i_{2}, \ldots, i_{d}\right\} \quad \text { and } \quad J=\left\{j_{1}, j_{2}, \ldots,\left[j_{d}\right]\right\}
$$

in which each term is a positive integer not equal to 1 (never $\infty$ ). The term $j_{d}$ is in brackets because, in some types, it is not present. Moreover, each label in (8.10.1) is the length label of $m$ consecutive columns of $A$ and $B$, namely the $m$ columns in the block over which it appears in the diagrams that define the individual forms.

As in Definition 8.7, we weave together the two label sequences $I$ and $J$ to form a single label sequence for each of the matrix pairs defined below. The two label sequences $I$ and $J$ reappear when we consider the associated matrix 4-tuples in Theorem 8.14 below.

We shall show [Theorem 8.14] that if $p$ is any of the "reduced" pairs below, then $f(p)$ is a self-conjugate block cycle 4-tuple. In the remaining case, cycle pairs, $f(p)$ is the direct sum of two conjugate, but non-self-conjugate, block cycle 4-tuples.

Bottom-bottom reduced pairs. Label $j_{d}$ occurs. We require:

(8.10.2) (i) $U \bar{U}^{-1}$ is indecomposable under similarity.

(ii) The concatenated sequence $\{I, \mu(J)\}$ is repetition-free; that is, it does not consist of repetitions of some strictly shorter sequence.

The form of these matrices is displayed in (8.10.3), below. Here $I=I_{m}$ with $m$ the block size. The matrix $A$ is a direct sum of one or more basic tiles (defined in Lemma 8.4), always with $Y=I$. The matrix $B$ is the direct sum of basic tiles (one fewer than $A$ ) and two exceptional tiles, one equal to the inverse $U^{-1}$ of the blocking matrix and the other equal to $I$. (We use the inverse $U^{-1}$ rather than the blocking matrix $U$ in the matrix $B$ in order to achieve uniformity in later formulas, for example, for $L$ in the first three parts of Theorem 8.14.) The basic tiles in $B$ always overlap those in $A$, as in the canonical forms that come from deleted cycle 4-tuples. Here, as before, entries not shown (except for ellipsis dots) are zero. As mentioned above, each term of the label sequences is the length label of all $m$ rows in the block over which it appears.

The extreme case is $d=1$, in which case $B$ contains no basic tiles. 
$(8.10 .3)$

$$
\begin{gathered}
A=\left[\begin{array}{ccccccccc}
i_{1} & j_{1} & i_{2} & j_{2} & i_{3} & \ldots & j_{d-1} & i_{d} & j_{d} \\
I & I & & & & \ldots & & & \\
I \varepsilon & I \bar{\varepsilon} & & & & \ldots & & & \\
& & I & I & & \ldots & & & \\
& & I \varepsilon & I \bar{\varepsilon} & & \ldots & & & \\
& & & & & \ddots & & & \\
& & & & & \ldots & & I & I \\
& & & & & \ldots & & I \varepsilon & I \bar{\varepsilon}
\end{array}\right] \\
B=\left[\begin{array}{lllllllll} 
& I & I & & & \ldots & & & \\
& I \varepsilon & I \bar{\varepsilon} & & & \ldots & & & \\
& & & I & I & \ldots & & & \\
& & & I \varepsilon & I \bar{\varepsilon} & \ldots & & & \\
& & & & & \ddots & & & \\
& & & & & \ldots & I & I & \\
& & & & & \ldots & I \varepsilon & I \bar{\varepsilon} & \\
I & & & & & \ldots & & & U^{-1} \\
& & & & & \ldots & & 0
\end{array}\right] .
\end{gathered}
$$

Bottom-top reduced pairs. Label $j_{d}$ does not occur. Again we require (8.10.2); the pair is as displayed below.

(8.10.4)

$$
\begin{aligned}
& i_{1} \quad j_{1} \quad i_{2} \quad j_{2} \quad i_{3} \quad \ldots \quad i_{d-1} j_{d-1} \quad i_{d} \\
& A=\left[\begin{array}{ccccccccc}
I & I & & & \ldots & & & \\
I \varepsilon & I \bar{\varepsilon} & & & \ldots & & & \\
& & I & I & \ldots & & & \\
& & I \varepsilon & I \bar{\varepsilon} & \ldots & & & \\
& & & & \ddots & & & \\
& & & & \ldots & I & I & \\
& & & & \ldots & I \varepsilon & I \bar{\varepsilon} & \\
& & & & & \ldots & & & U
\end{array}\right]
\end{aligned}
$$

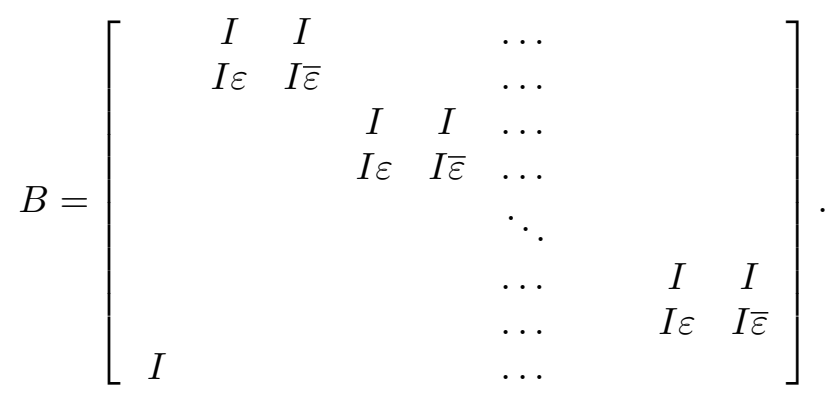

In the extreme case $d=1$ no basic tiles occur. 
Top-top-reduced pairs. These are the same as bottom-bottom-reduced pairs except that $A$ is interchanged with $B$ and the matrix $U^{-1}$ is replaced by the blocking matrix $U$. In particular, we require (8.10.2).

Cycle pairs. Label $j_{d}$ occurs. Here we require:

(8.10.5) (i) The blocking matrix $U$ is indecomposable under similarity.

(ii) The blocking matrix $U$ is not similar to $\bar{U}^{-1}$ or, for all cycles $\nu^{t}$, $J \neq \nu^{t} \mu(I)$, where $\nu$ is the unit forward rotation and $\mu$ is the mirror image permutation [Notation 8.6].

(iii) The sequence of pairs $\left(i_{1}, j_{1}\right), \ldots,\left(i_{d}, j_{d}\right)$ is repetition-free.

These pairs have the form displayed in diagram (8.10.6) below. Thus, no exceptional tiles occur here. Note that both $A$ and $B$ are direct sums of basic tiles, but one of the tiles "wraps" from the end to the begining of $B$. The blocking matrix $U$ always occurs in exactly one basic tile: The tile in the upper left corner of $A$.

$(8.10 .6)$

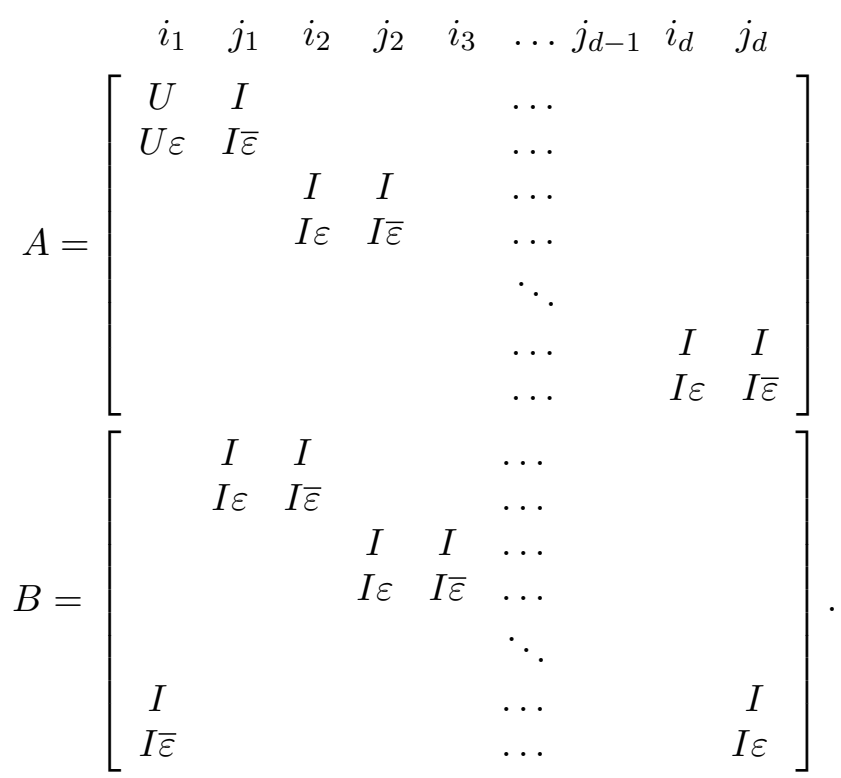

In order to prove that our list of canonical forms yields all isomorphism classes of self-conjugate 4-tuples, we need the following matrix version of Hilbert's Theorem 90. It follows immediately from Ballantine [Ba, Theorem 2.1], using the fact that every matrix over a field is similar to its transpose.

Lemma 8.11 ("Theorem 90" for matrices). Let $F$ be a 2-dimensional separable extension field of a field $k$. Then the following two assertions about an invertible matrix $L$ over $F$ are equivalent.

(i) $L$ is similar to $\bar{L}^{-1}$.

(ii) $L$ is similar to $U \bar{U}^{-1}$ for some invertible symmetric matrix $U$ over $F$. 
Also, we need to determine the block cycle 4-tuple that is isomorphic to the conjugate of a given block cycle 4-tuple.

Lemma 8.12. Let $g$ and $h$ be the block cycle 4-tuples with blocking matrices $L$ and $\bar{L}^{-1}$ respectively, and with label sequences:

$$
\begin{aligned}
& g: \quad H=\left\{i_{1}, i_{2}, \ldots, i_{e}\right\} \\
& h: \mu(K)=\left\{j_{e}, j_{e-1}, \ldots, j_{1}\right\} \text { and } \mu(H)=\left\{i_{e}, i_{e-1}, \ldots, i_{1}\right\} \text {. }
\end{aligned}
$$

Then $\bar{g} \cong h$. In particular, $g \cong \bar{g}$ if and only if $L$ is similar to $\bar{L}^{-1}$ and $K=\nu^{t} \mu(H)$ (equivalently, $H=\nu^{t} \mu(K)$ ) for some cycle $\nu^{t}$.

Proof. Let the size of $L$ be $m \times m$. We have $g=(A, I, P, I)$ where, as displayed in (7.2.4) with $d$ in place of the present $e, A$ is the me $\times m e$ block diagonal matrix whose upper left main-diagonal block is $L$ and whose other main diagonal blocks equal $I_{m}$, and $P$ is a block-permutation matrix. Then $\bar{g}=(I, \bar{A}, I, P)$, where the length labels are moved in exactly the same way that the matrices were moved. Let $g^{\prime}$ be obtained from $\bar{g}$ by reversing the order of the $m$-column blocks in all four matrices - together with their length labels — but keeping the columns within each $m$-column block in their original order in order not to disturb the blocking matrix. Since this is a display operation we have $g^{\prime} \cong \bar{g}$ [Notation 6.9 and Theorem 6.10].

Let $R$ be the block permutation matrix whose $e$ nonzero blocks each equal $I_{m}$, and are located on the diagonal that runs from the lower left corner to the upper right corner of $R$. Then we have $g^{\prime}=(R, \bar{A} R, R, P R)$, and the label sequences for $g^{\prime}$ are those of $h$, displayed in (8.12.1). Let $g^{\prime \prime}$ be the 4tuple obtained by left-multiplying all four matrices of $g^{\prime}$ by $R$. Then $g^{\prime \prime} \cong g^{\prime}$ by Theorem 6.7 , and we have $g^{\prime \prime}=(I, R \bar{A} R, I, R P R)$.

Let $g^{\prime \prime \prime}$ be obtained by left multiplying the first and second matrices of $g^{\prime \prime}$ by $(R \vec{A} R)^{-1}$ and the third and fourth matrices by $(R P R)^{-1}$. Then $g^{\prime \prime \prime} \cong g^{\prime \prime}$ and we have $g^{\prime \prime \prime}=\left(R \bar{A}^{-1} R, I, R P^{-1} R, I\right)$.

Note that $R P^{-1} R=P$. (This is essentially formula (8.6.1).)

Next consider the form of $R \bar{A} R$. $\bar{A}$ is the block diagonal matrix whose diagonal blocks are $\bar{L}, I, I, \ldots, I$. Therefore $R \bar{A}^{-1} R$ is the block diagonal matrix whose diagonal blocks are $I, I, \ldots, I, \bar{L}^{-1}$. Thus, when $e=5$, for example, our 4-tuple $g^{\prime \prime \prime}$ is as shown in (8.12.2) below.

Therefore, to complete the proof it now suffices to move $\bar{L}^{-1}$ to the upper left corner of $A_{1}$. This is accomplished by the following sequence of multiplications, which are $F-F$ sweeping-similarity operations: Left multiply the last row block of $A_{1}$ and $A_{2}$ by $\bar{L}$; then right multiply the $i_{1}$-labeled columns of $A_{2}$ and $B_{2}$ by $\bar{L}^{-1}$; then left multiply the last row block of $B_{1}$ and $B_{2}$ by $\bar{L}$; then right multiply the $j_{5}$-labeled columns of $A_{1}$ and $B_{1}$ by $\bar{L}^{-1}$. The resulting matrix 4-tuple is the given $h$, and so $h \cong \bar{g}$. 
$(8.12 .2)$

\begin{tabular}{|c|c|c|c|c|c|c|c|c|c|c|c|}
\hline & $j_{5}$ & $j_{4}$ & $j_{3}$ & $j_{2}$ & $j_{1}$ & & $i_{5}$ & $i_{4}$ & $i_{3}$ & $i_{2}$ & $i_{1}$ \\
\hline \multirow{5}{*}{$A_{1}=$} & $I$ & 0 & 0 & 0 & 0 & \multirow{5}{*}{$A_{2}=$} & $I$ & 0 & 0 & 0 & 0 \\
\hline & 0 & $I$ & 0 & 0 & 0 & & 0 & $I$ & 0 & 0 & 0 \\
\hline & 0 & 0 & $I$ & 0 & 0 & & 0 & 0 & $I$ & 0 & 0 \\
\hline & 0 & 0 & 0 & $I$ & 0 & & 0 & 0 & 0 & $I$ & 0 \\
\hline & 0 & 0 & 0 & 0 & $\bar{L}^{-1}$ & & 0 & 0 & 0 & 0 & $I$ \\
\hline \multirow{5}{*}{$B_{1}=$} & 0 & $I$ & 0 & 0 & 0 & \multirow{5}{*}{$B_{2}=$} & $I$ & 0 & 0 & 0 & 0 \\
\hline & 0 & 0 & $I$ & 0 & 0 & & 0 & $I$ & 0 & 0 & 0 \\
\hline & 0 & 0 & 0 & $I$ & 0 & & 0 & 0 & $I$ & 0 & 0 \\
\hline & 0 & 0 & 0 & 0 & $I$ & & 0 & 0 & 0 & $I$ & 0 \\
\hline & $I$ & 0 & 0 & 0 & 0 & & 0 & 0 & 0 & 0 & $I$ \\
\hline
\end{tabular}

Supplementary statement. Suppose that $g \cong \bar{g}$. By the part of the lemma already proved, $\bar{g}$ is isomorphic to a block cycle 4 -tuple with blocking matrix $\bar{L}^{-1}$ and pair of label sequences $\mu(K)$ and $\mu(H)$. But by the uniqueness theorem for block cycle 4-tuples [Theorem 7.3], the pair of label sequences of any block cycle 4-tuple isomorphic to $g$ must have the form $\nu^{t}(H)$ and $\nu^{t}(K)$ for some cycle $\nu^{t}$. This yields $\nu^{t}(K)=\mu(H)$, and hence $K=\nu^{-t} \mu(H)=$ $\mu \nu^{-t}(H)$, as desired. Moreover, also by the uniqueness theorem for block cycle 4 -tuples, the blocking matrix $\bar{L}^{-1}$ of $\bar{g}$ must be similar to the blocking matrix $L$ of $g$.

Conversely, suppose that $L$ is similar to $\bar{L}^{-1}$ and $K=\nu^{t} \mu(H)$ for some cycle $\nu^{t}$. Then formula (8.6.1) yields $H=\nu^{t} \mu(K)$. Therefore, by the uniqueness theorem for block cycle 4-tuples [Theorem 7.3], the block cycle 4-tuple $g$, with blocking matrix $L$ and label sequences $H$ and $K$, is isomorphic to the block cycle 4 -tuple with blocking matrix $L$ and label sequences $\nu^{-t}(H)=\mu(K)$ and $\nu^{-t}(K)=\mu(H)$, which is in turn isomorphic to $\bar{g}$, since $L$ is similar to $\bar{L}^{-1}$.

The following lemma simplifies the way to visualize self-conjugate block cycle 4-tuples.

Lemma 8.13. Let $g$ be a self-conjugate block cycle 4-tuple. Then $g$ is isomorphic to a block cycle 4-tuple whose pair of label sequences has one of the forms:

(a) $H$ and $\mu(H)$, or

(b) $H$ and $\nu \mu(H)$

where $\mu$ is the mirror image permutation and $\nu$ is the unit forward rotation.

Let $e$ be the number of terms in $H$. If $e$ is even then only one of (a) and (b) applies to $\mathrm{g}$. If $e$ is odd then (a) always applies. 
Proof. By Lemma 8.12, $g$ has a pair of label sequences of the form $H$ and $K=\nu^{t} \mu(H)$ for some cycle $\nu^{t}$. We consider two cases, according to the parity of $t$.

Case (a): $t=2 s$ for some integer $s$. Then $K=\nu^{2 s} \mu(H)=\nu^{s} \mu \nu^{-s}(H)$ and hence $\nu^{-s}(K)=\mu \nu^{-s}(H)$. Therefore, by the uniqueness theorem for block cycle 4-tuples, possibility (a) holds if we replace $H$ and $K$ by the sequences $\nu^{-s}(H)$ and $\nu^{-s}(K)$, respectively.

Case (b): $t=2 s+1$ for some integer $s$. Proceed similarly to case (a).

Now suppose that $e$ is odd. Then the cyclic group generated by $\nu$ has odd order, and therefore contains a square root of $\nu$. Say $\nu=\left(\nu^{u}\right)^{2}$. Then $\nu^{t}=\nu^{2 u t}$ and case (a) applies.

Finally, suppose (by way of contradiction) that $e$ is even and both possibilities (a) and (b) hold. Thus some pair of isomorphic block cycle 4-tuples have label sequences of the form $H$ and $\mu(H)$, and $H^{\prime}$ and $\nu \mu\left(H^{\prime}\right)$, respectively. By the uniqueness theorem for block cycle 4-tuples, there is a cycle $\nu^{t}$ such that $\nu^{t}(H)=H^{\prime}$ and $\nu^{t} \mu(H)=\nu \mu\left(H^{\prime}\right)$. Therefore, $\nu^{t} \mu(H)=\nu \mu \nu^{t}(H)$. Simplifying, using (8.6.1), yields $H=\nu^{2 t-1}(H)$. Since $e$ is even, $\nu^{2 t-1} \neq 1$. Thus $H$ consists of some number, say $r$, of repetitions of some strictly shorter sequence, and hence so does $\mu(H)$. But then the sequence of pairs of labels from $H$ and $\mu(H)$ is not repetition-free, contrary to the definition of a block cycle 4 -tuple [see (7.2.3)(i)].

Theorem 8.14. Let $p=(A, B)$ be any of the types of matrix pairs listed below, with blocking matrix $U$ and constructed from the pair of label sequences

$$
I=\left\{i_{1}, \ldots, i_{d-1}, i_{d}\right\} \quad \text { and } \quad J=\left\{j_{1}, \ldots, j_{d-1},\left[j_{d}\right]\right\} .
$$

Then $f(p)$ is as described below, where $\mu$ is the mirror image permutation, and $\nu$ is the unit forward rotation, as in Notation 8.6.

(i) ( $p$ bottom-bottom reduced): Then $f(p)$ is isomorphic to the self-conjugate block cycle 4-tuple whose blocking matrix is $L=U \bar{U}^{-1}$ and whose pair of label sequences is $\{I, \mu(J)\}$ and $\{J, \mu(I)\}$.

(ii) ( $p$ bottom-top reduced): Then $f(p)$ is isomorphic to the self-conjugate block cycle 4-tuple whose blocking matrix is $L=U \bar{U}^{-1}$ and whose pair of label sequences is $\{I, \mu(J)\}$ and $\{J, \mu(I)\}$. (Recall that, in this case, label $j_{d}$ does not occur.)

(iii) ( $p$ top-top reduced): Then $f(p)$ is isomorphic to the self-conjugate block cycle 4-tuple whose blocking matrix is $L=U \bar{U}^{-1}$ and whose pair of label sequences is $\{J, \mu(I)\}$ and $\nu(\{I, \mu(J)\})$.

(iv) ( $p$ a cycle pair): Then $f(p)$ is isomorphic to the direct sum $g \oplus \bar{g}$ where $g$ is the non-self-conjugate block cycle 4-tuple whose blocking matrix is $L=U$ and whose pair of label sequences is $I$ and $J$. 
In all cases, $p$ is an indecomposable matrix pair (equivalently, $f(p)$ is an indecomposable package). Conversely, if $g$ is a non-self-conjugate block cycle 4-tuple, then there is some cycle pair $p$ with $f(p) \cong g \oplus \bar{g}$, and if $g$ is a self-conjugate block cycle 4-tuple, then there is some bottom-bottom reduced, bottom-top reduced or top-top-reduced matrix pair $p$ with $f(p) \cong g$.

Proof. Write $f(p)=(A, \bar{A}, B, \bar{B})$. We do situation (i) in detail, and then the others more briefly. Situation (i) itself is a slightly more complicated version of what was done in the deleted cycle case, and we refer to that case for steps that are very similar.

(i) $p$ is bottom-bottom reduced, as in (8.10.3). As in the deleted cycle situations we first reduce every pair of basic tiles, as described in Lemma 8.4, and call the resulting 4 -tuple $h$. The situation $d=3$ is shown in (8.14.2).

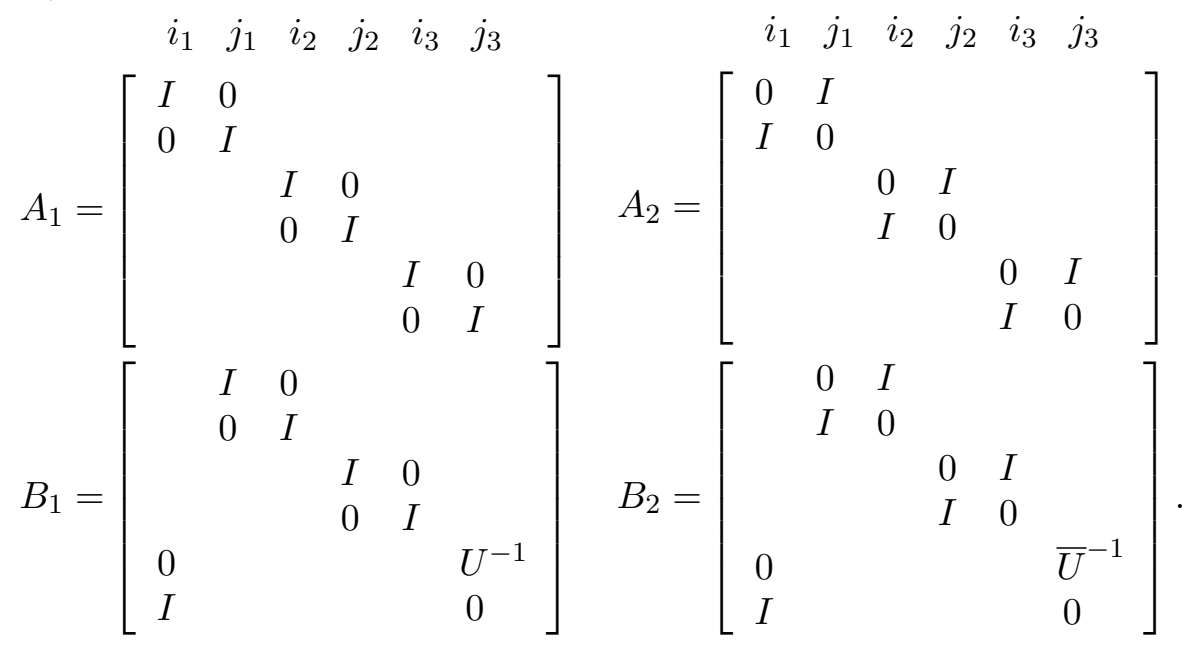

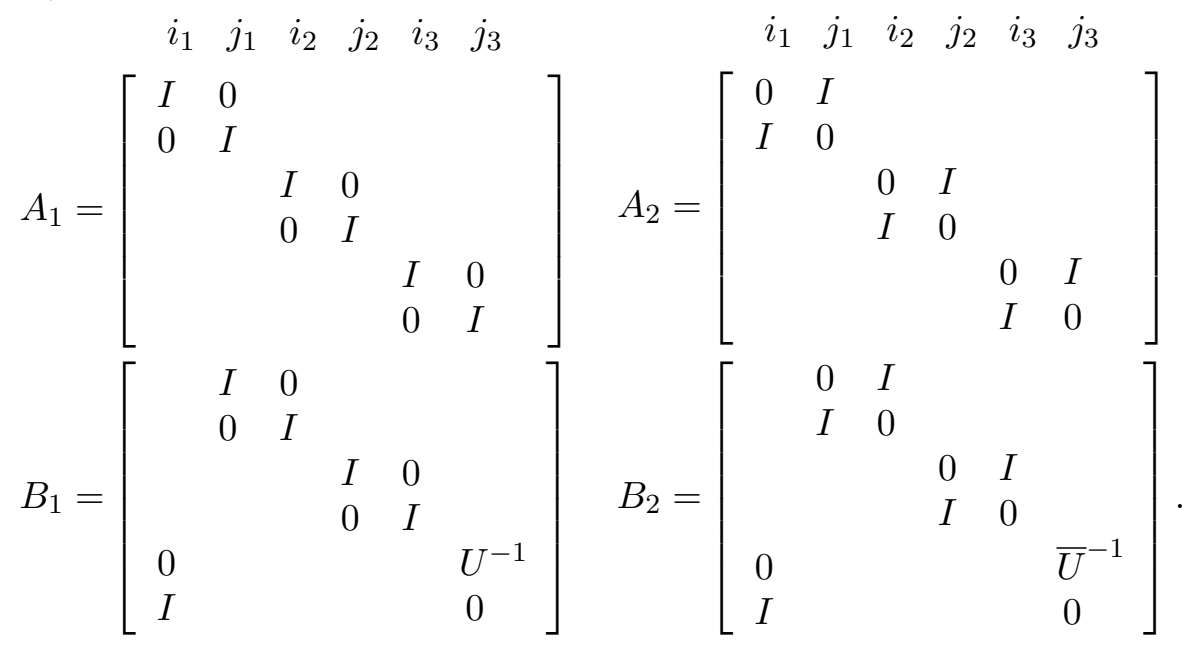

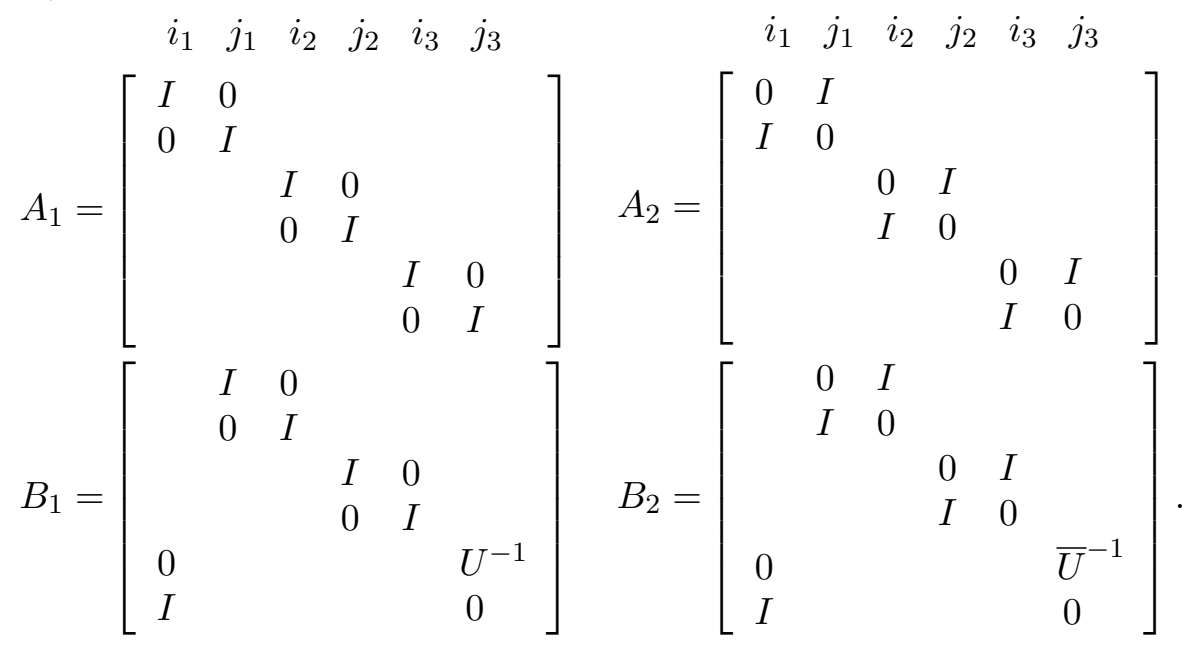

Note that statement (8.9.3) is valid here, too if we replace "column" by "m-column block," and it obviously remains valid after arbitrary display operations. Apply the display operation - in block form - that changes the pair of label sequences in (8.14.2) to the desired sequences $\{I, \mu(J)\}$ and $\{J, \mu(I)\}$, and call the resulting 4-tuple $h^{\prime}$. By (8.9.3) we have $h^{\prime}=$ $\left(C, C, B_{1}^{\prime}, B_{2}^{\prime}\right)$ for some $C$. Left multiplying the $A$-matrices of $h^{\prime}$ by $C^{-1}$ converts it to $h^{\prime \prime}=\left(I, I, B_{1}^{\prime}, B_{2}^{\prime}\right)$.

Next, change the pair $\left(U^{-1}, \bar{U}^{-1}\right)$ to $\left(\bar{U} U^{-1}, I\right)=\left(L^{-1}, I\right)$, by left-multiplying the row-block containing these matrices by $\bar{U}$. Then right-multiply the $j_{d}$-labeled columns of $A_{1}$ and $B_{1}$ by $L$, changing $L^{-1}$ to $I$ and changing the corresponding nonzero entry in the $j_{d}$-labeled column-block of $A_{1}$ to $L$. The result of this is that, after a change of notation, $h^{\prime \prime}$ takes the form $\left(D, I, B_{1}, B_{2}\right)$ where $D$ is a block-diagonal matrix one of whose diagonal 
blocks equals $L$ and whose other diagonal entries equal $I$, and $B_{1}$ and $B_{2}$ are block-permutation matrices.

Now consider the pair of block-permutation matrices $\left(B_{1}, B_{2}\right)$. We can arbitrarily permute the row blocks of this pair by simultaneously left-multiplying $B_{1}$ and $B_{2}$ by a suitable permutation matrix. Multiply by $B_{2}^{-1}$, so that $B_{2}$ is replaced by an identity matrix. The result of all of the above transformations is illustrated below, in the case $d=3$. (Ignore the entries of $B_{1}$ until we determine them.)

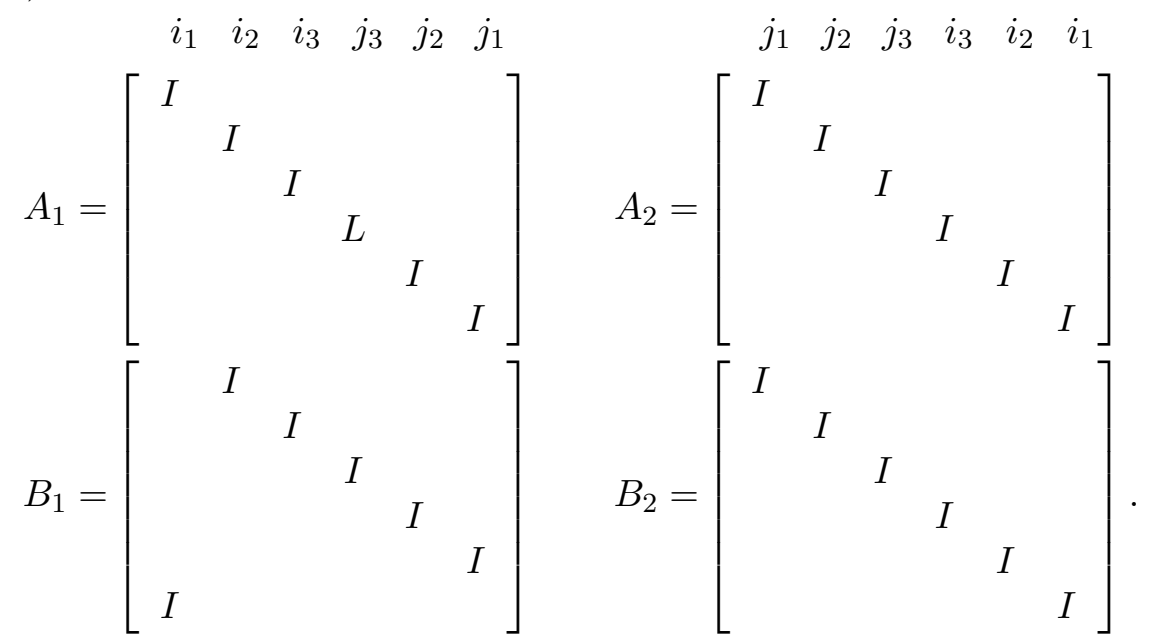

Next we find the locations of the nonzero entries of $B_{1}$. First look for the nonzero entries of the first three row-blocks. Consider column-blocks $j_{1}, j_{2}$, and $j_{3}$ of $B_{2}$ in (8.14.3). We ask: Which column-blocks of $B_{1}$ have their nonzero entries in the same rows as the nonzero entries of the $j_{1}, j_{2}$, $j_{3}$-labeled column-blocks of $B_{2}$ ? The answer - if we identify columns by their formal labels $j_{\nu}$ - is unchanged by simultaneous row permutations in $B_{1}, B_{2}$ and by arbitrary display operations. After consulting (8.14.2) we see that we want the $i_{2}, i_{3}$, and $j_{3}$-labeled column-blocks of $B_{1}$. Thus, returning to (8.14.3), we see that the first three row-blocks of $B_{1}$ have their nonzero entries in the second, third, and fourth column-blocks of $B_{1}$, respectively, as shown. By proceeding in this fashion, we eventually find that $B_{1}$ is the block form of the cyclic permutation matrix required for a block cycle 4-tuple.

In order to complete the proof, two things are needed: (a) Move $L$ to the upper left corner of $A_{1}$; and (b) check that the sequence of pairs of labels from $\{I, \mu(J)\}$ and $\{J, \mu(I)\}$ is repetition-free (as required in the definition of a block cycle 4-tuple).

(a) Move $L$. It suffices to slide $L$ up one level; that is, move it to the next higher $I$, for we can repeat this often enough to get $L$ to the right location. We accomplish this by the following four sweeping-similarity operations. 
Right-multiply the column-block of $A_{1}$ and $B_{1}$ containing $L$ by $L^{-1}$, thus replacing the nonzero entry in that column-block of $B_{1}$ by $L^{-1}$. Then use a left multiplication of the row block of $\left(B_{1}, B_{2}\right)$ containing $L^{-1}$ to put $L$ into that row-block of $B_{2}$. Then use a right multiplication to put $L^{-1}$ into $A_{2}$, and finally a left multiplication to put $L$ into $A_{1}$.

(b) By hypothesis (8.10.2)(ii), the sequence $\{I, \mu(J)\}$ is repetition-free, which is formally stronger than the statement that the sequence of pairs of labels from $\{I, \mu(J)\}$ and $\{J, \mu(I)\}$ is repetition-free. (Actually, since the sequence $\{J, \mu(I)\}$ is just $\{I, \mu(J)\}$ written backwards, the two types of "repetition-free" are equivalent here.)

(ii) $p$ is bottom-top reduced, as in (8.10.4). We omit the details of this reduction, which is similar to that in (i).

(iii) $p$ is top-top reduced. Thus the matrix pair $p$ is obtained from the bottom-bottom reduced pair $p^{\prime}$ in (8.10.3) by interchanging $A$ with $B$ and replacing $U^{-1}$ by $U$. All of steps in the reduction of $f\left(p^{\prime}\right)$ done in part (i) can be done with the roles of $A$ and $B$ reversed. Therefore, by the result of (i), $f(p)$ is isomorphic to the first matrix 4-tuple displayed in (8.14.4) with its pair of label sequences above it.

$$
\begin{array}{cccccccc}
\{I, \mu(J)\} & \{J, \mu(I)\} & & \{J, \mu(I)\} & \{I, \mu(J)\} & & \nu^{-1}\{J, \mu(I)\} & \{I, \mu(J)\} \\
P & I & \rightarrow & R P R & I & \rightarrow & I & I \\
D & I & & R D R & I & & R D R P & I .
\end{array}
$$

Here $P$ is the block permutation matrix displayed as $B_{1}$ in (7.2.4). This matrix, acting on the right performs the inverse $\nu^{-1}$ of the unit forward column rotation. The matrix $D$ is a block diagonal matrix (see $A_{1}$ in (7.2.4)) whose upper left-hand block is $U^{-1} \bar{U}$ and whose other diagonal blocks each equal $I_{m}$, by the result of part (i) with $U$ in place of $U^{-1}$.

Let $R$ ("reversal matrix") be the matrix formed by reversing the order of the columns of the $d \times d$ identity matrix and then replacing each nonzero entry by $I_{m}$ and each zero entry by a zero matrix.

To continue the reduction of $f(p)$, perform the display operation that reverses the order of the column blocks of $P$ and $D$, together with their length labels, while leaving the order of the columns within each block unchanged; and do the same with the two identity matrices. Then left-multiply all 4 matrices by $R$, obtaining the second displayed 4-tuple.

Note that $R P R=P^{-1}$ (this is essentially formula (8.6.1)). Therefore right-multiplying the left-hand pair of matrices and its row of column labels by $P$ is a display operation. It yields the third 4-tuple in (8.14.4).

Note that $R D R=D_{1}$, the block diagonal matrix obtained by moving its nonidentity bock $U^{-1} \bar{U}$ to the lower right-hand corner. Moreover, $D_{1} P=$ $P D_{2}$, where $D_{2}$ is the block diagonal matrix obtained from $D_{1}$ by moving $U^{-1} \bar{U}$ to a different block on the main diagonal. Right-multiplying this column-block of $P D_{2}$ and the identity matrix above it by $\left(U^{-1} \bar{U}\right)^{-1}$ is a 
sweeping-similarity operation and replaces the last 4-tuple in (8.14.4) by $\left(D_{2}^{-1}, I, P, I\right)$ without moving any length-labels. Note that $D_{2}^{-1}$ is the block diagonal matrix obtained from $D_{2}$ by inverting its nonidentity diagonal block $U^{-1} \bar{U}$. This diagonal block of $D_{2}^{-1}$ therefore equals $\bar{U}^{-1} U$.

Call this last 4-tuple $h^{\prime}$. Then $h^{\prime}$ is the block cycle 4-tuple in the statement of the theorem except that: (a) Its blocking matrix is in the wrong block of $A_{1}$; (b) the blocking matrix of $h$ is $\bar{U}^{-1} U$ instead of $U \bar{U}^{-1}$; and (c) the pair of label sequences is not yet exactly correct.

(a) As in the proof of part (i) ["Move $L$ "] we can slide $\bar{U}^{-1} U$ up to the upper left corner of $A_{1}$ without changing the isomorphism class of the 4tuple. The resulting 4 -tuple is now a block cycle 4 -tuple (though not yet the desired one).

(b) Reversing factors in a product of two invertible matrices does not change its similarity class, and replacing the blocking matrix of a block cycle 4-tuple within its similarity class does not change the isomorphism class of the 4-tuple [Theorem 7.3(ii)]. Doing this yields the correct blocking matrix. Call this latest 4-tuple $h^{\prime \prime}$.

(c) Performing any rotation simultaneously to both of the label sequences of any block cycle matrix leaves the isomorphism class of the matrix unchanged [Theorem 7.3(ii)]. Note that the pair of label sequences of $h^{\prime \prime}$ is the same as that of the last 4-tuple displayed in (8.14.4). Therefore applying the rotation $\nu$ to these label sequences yields the desired sequences, and completes the proof of (iii).

(iv) $p$ is a cycle pair, as in (8.10.6). Unlike the previous cases, the reduction of basic tiles in this case uses the full strength of Lemma 8.4, because $U$ is located in a basic tile. Otherwise, the details are just a slight modification of the bottom-bottom reduced case, and we omit them. As in the nonreduced deleted cycle situation, the reduction in this case actually proves:

(8.14.5) We have $f(p) \cong g \oplus \bar{g}$, even without the nonsymmetry restriction $(8.10 .5)$ (ii) in the definition of a cycle pair.

Supplementary statements. Indecomposability is an immediate consequence of Lemma 8.5.

To see that our list is exhaustive, let $g$ be any block cycle 4-tuple, with blocking matrix $L$ and pair of label sequences $I^{\prime}$ and $J^{\prime}$.

Consider, first, the case that $g \cong \bar{g}$. Then $L$ is similar to $\bar{L}^{-1}$ [Lemma 8.12]. Therefore, by Ballantine's Hilbert Theorem 90 [Lemma 8.11], there is a matrix $U$ such that $U \bar{U}^{-1}$ is similar to $L$. This gives us the blocking matrix $U$ to use in situations (i)-(iii) of the theorem. Again, since $g \cong \bar{g}$, we can choose $g$, within its isomorphism class, such that $J^{\prime}=\mu\left(I^{\prime}\right)$ or $J^{\prime}=\nu \mu\left(I^{\prime}\right)$ [Lemma 8.13]. Moreover, if the length $e$ of each of these sequences is odd, 
then we can choose $J^{\prime}=\mu\left(I^{\prime}\right)$. This yields three cases, corresponding to the enumeration of situations in the statement of the theorem:

Case (i): $e$ is even and $J^{\prime}=\mu\left(I^{\prime}\right)$. Since $e$ is even we can define sequences $I$ and $J$ of length $d=e / 2$ by $I^{\prime}=\{I, \mu(J)\}$. Take $p$ to be the bottom-bottom reduced pair of (i), with blocking matrix $U$ and label sequences $I$ and $J$. Then the block cycle 4 -tuple yielded by situation (i) has blocking matrix $L$ and label sequences $\{I, \mu(J)\}=I^{\prime}$ and $\{J, \mu(I)\}=\mu\left(I^{\prime}\right)=J^{\prime}$, as desired.

Case (ii): $e$ is odd and $J^{\prime}=\mu\left(I^{\prime}\right)$. Since $e$ is odd, we can define sequences $I$ and $J$ of lengths that we call $d$ and $d-1$ respectively, such that $I^{\prime}=$ $\{I, \mu(J)\}$. Take $p$ to be the bottom-top reduced pair of (ii), with blocking matrix $U$ and label sequences $I$ and $J$. Then the block cycle 4 -tuple yielded by situation (ii) has blocking matrix $L$ and the desired label sequences $I^{\prime}$ and $J^{\prime}$, as in Case (i).

Case (iii): $e$ is even and $J^{\prime}=\nu \mu\left(I^{\prime}\right)$. Define sequences $I$ and $J$ of length $d=e / 2$ by $I^{\prime}=\{J, \mu(I)\}$. Take $p$ to be the top-top reduced pair of (iii), with blocking matrix $U$ and label sequences $I$ and $J$. Then the block cycle 4-tuple yielded by situation (iii) has blocking matrix $L$ and label sequences $I^{\prime}=\{J, \mu(I)\}$ and $\nu(\{I, \mu(J)\})=\nu \mu\left(I^{\prime}\right)=J^{\prime}$, as desired.

This leaves the case that $g$ is non-self-conjugate; and here we take $p$ to be the cycle pair of (iv).

Theorem 8.15. Let $g$ be a matrix 4-tuple. The following are equivalent:

(i) $g$ is a package.

(ii) $g \cong \bar{g}$.

(iii) $g \cong \oplus_{i} f\left(p_{i}\right)$, where each $p_{i}$ is one of the seven canonical forms in Definitions 8.7 and 8.10 .

Proof. We have already noted that (i) $\Rightarrow$ (ii) follows from the definitions. Also, (iii) $\Rightarrow$ (i) is immediate since each $f\left(p_{i}\right)$ is a package.

Thus, the only nontrivial implication is (ii) $\Rightarrow$ (iii), so suppose $g \cong \bar{g}$. By the structure theorems for decompositions of 4-tuples [Theorems 7.3 and 7.4] we have

$$
g \cong \oplus_{j} g_{j} \quad \text { and therefore } \quad \bar{g} \cong \oplus_{j} \bar{g}_{j}
$$

where each $g_{j}$ is either a deleted or block cycle 4-tuple, and the multiplicity of each isomorphism class of deleted or block cycle 4-tuple that occurs in the first decomposition is determined by the isomorphism class of $g$. Since the conjugate of every deleted cycle or block cycle 4-tuple is again (isomorphic to) a deleted or block cycle 4-tuple, respectively [Lemmas 8.8 and 8.12] and $g \cong \bar{g}$, it follows from Theorem 7.4 that, for each index $j, \bar{g}_{j}$ occurs (up to isomorphism) in the decomposition (8.15.1) of $g$ exactly as often as $g_{j}$ 
occurs. Therefore $g$ has a decomposition of the form

$$
g \cong \oplus_{i} h_{i}
$$

where each $h_{i}$ is either a self-conjugate $g_{j}$ or the direct sum $g_{j} \oplus \bar{g}_{j}$ for some $g_{j}$ such that $g_{j} ¥ \bar{g}_{j}$. Each $h_{i}$ is therefore isomorphic to $f\left(p_{i}\right)$ for some deleted or block cycle pair $p_{i}$ by Theorems 8.9 and 8.14 , completing the proof.

Corollary 8.16. Suppose $g$ is a matrix 4-tuple. Then $g$ is an indecomposable package if and only if $g$ is either:

(i) A self-conjugate, indecomposable 4-tuple; or

(ii) The direct sum of two conjugate, non-self-conjugate, indecomposable 4-tuples.

Proof. This follows from Theorem 8.15 and the fact that, for all of our canonical forms $p, f(p)$ has the form (i) or (ii) [Theorems 8.9 and 8.14].

Corollary 8.17. Every indecomposable matrix pair is isomorphic to one of the seven canonical forms in Definitions 8.7 and 8.10. Moreover, if

$$
\oplus_{i=1}^{m} p_{i} \cong \oplus_{j=1}^{n} q_{j}
$$

where each $p_{i}$ and each $q_{j}$ is indecomposable, then $m=n$ and, after suitable renumbering, $p_{i} \cong q_{i}$ for each index $i$.

Proof. If $p$ is a matrix pair, then $f(p)$ is self-conjugate, so by Theorem 8.15, we can write $f(p) \cong \oplus_{i} f\left(p_{i}\right)$, where each $p_{i}$ is a one of our canonical forms. By Theorem 8.3, it follows that $p \cong \oplus_{i} p_{i}$, from which the first assertion of the corollary follows.

For the uniqueness claim, suppose that we are given an isomorphism of direct sums as in (8.17.1). Then by Theorem 8.3 we get that $\oplus_{i=1}^{m} f\left(p_{i}\right) \cong$ $\oplus_{j=1}^{n} f\left(q_{j}\right)$. It suffices to show that some $q_{i}$, say $q_{1}$, satisfies $p_{1} \cong q_{1}$ and $\oplus_{i \neq 1} p_{i} \cong \oplus_{i \neq 1} q_{i}$. Write each $f\left(p_{i}\right)$ and $f\left(q_{j}\right)$ as the direct sum of one or two indecomposable terms, as described in Theorems 8.9 and 8.14.

Let $x$ be one of the one or two indecomposable direct summands of $f\left(p_{1}\right)$. By the Krull-Schmidt Theorem for 4-tuples [Theorem 7.4], some indecomposable direct summand on the right-hand side, say of $f\left(q_{1}\right)$, must be isomorphic to $x$. If $x$ is not a package, then $x \oplus \bar{x}$ is the unique indecomposable package containing $x$ as a direct summand [by Corollary 8.16], and is therefore isomorphic to both $f\left(p_{1}\right)$ and $f\left(q_{1}\right)$. Otherwise $x$ itself is a package, and indecomposability of $f\left(p_{1}\right)$ and $f\left(q_{1}\right)$ again implies that $f\left(p_{1}\right) \cong f\left(q_{1}\right)$. In either case the Krull-Schmidt Theorem for 4-tuples then implies that $\oplus_{i \neq 1} f\left(p_{i}\right) \cong \oplus_{i \neq 1} f\left(q_{i}\right)$, and therefore $p_{1} \cong q_{1}$ and $\oplus_{i \neq 1} p_{i} \cong \oplus_{i \neq 1} q_{i}$ [Theorem 8.3], completing the proof. 
There remains the question of uniqueness of the seven canonical forms in Definitions 8.7 and 8.10. The next theorem establishes the extent of this uniqueness.

Theorem 8.18. Let $p$ and $q$ be matrix pairs in canonical form, with pairs of label sequences $I$ and $J$, and $I^{\prime}$ and $J^{\prime}$, respectively, and blocking matrices $U$ and $V$, respectively, if relevant. Then $p \cong q$ if and only if they are of the same type (i)-(vii) below, and the conditions listed for their type hold (with $\mu$ and $\nu$ as in Notation 8.6).

(i) (Nonreduced pair): Either $I^{\prime}=I$ and $J^{\prime}=J$, or $I^{\prime}=\mu(J)$ and $J^{\prime}=\mu(I)$.

(ii) (Bottom-reduced pair): $I^{\prime}=I$ and $J^{\prime}=J$.

(iii) (Top-reduced pair): $I^{\prime}=I$ and $J^{\prime}=J$.

(iv) (Bottom-bottom reduced pair): Either $I^{\prime}=I$ and $J^{\prime}=J$, or $I^{\prime}=\mu(J)$ and $J^{\prime}=\mu(I)$; and (in either case) $V \bar{V}^{-1}$ is similar to $U \bar{U}^{-1}$.

(v) (Bottom-top reduced pair): $I^{\prime}=I$ and $J^{\prime}=J$; and $V \bar{V}^{-1}$ is similar to $U \bar{U}^{-1}$.

(vi) (Top-top reduced pair): Either $I^{\prime}=I$ and $J^{\prime}=J$, or $I^{\prime}=\mu(J)$ and $J^{\prime}=\mu(I)$; and (in either case) $V \bar{V}^{-1}$ is similar to $U \bar{U}^{-1}$.

(vii) (Cycle pair): Either $I^{\prime}=\nu^{t}(I)$ and $J^{\prime}=\nu^{t}(J)$ (for some $t$ ) and $V$ is similar to $U$; or $I^{\prime}=\nu^{t} \mu(J)$ and $J^{\prime}=\nu^{t} \mu(I)$ (for some $t$ ) and $V$ is similar to $\bar{U}^{-1}$.

Proof. All of the cases considered in this proof use our description of $f(p)$ in terms of $p$ [Theorems 8.9 and 8.14] and the fact that $p \cong q \Longleftrightarrow f(p) \cong f(q)$ [Theorem 8.3].

Uniqueness of types: We claim that the type, (i)-(vii), of $p$ is determined by the isomorphism class of $p$. It suffices to show that this type is determined by the isomorphism class of $f(p)$. We use the following facts: $f(p)$ is the direct sum of one or two deleted cycle or block cycle 4-tuples; deleted cycle and block cycle 4-tuples are indecomposable [Theorem 7.3]; the KrullSchmidt Theorem holds for direct sums of 4-tuples [Theorem 7.4]; and no deleted cycle 4-tuple is isomorphic to any block cycle 4-tuple [Theorem 7.3]. The following enumeration of cases therefore distinguishes among types of 4-tuples.

Types (i)-(iii) are the types for which $f(p)$ is a direct sum of deleted cycle 4-tuples. Among these types, Type (i) yields two summands while Types (ii) and (iii) each yield only one summand. In both of these last two types the first label sequence of $f(p)$ is $\{I, \mu(J)\}$. In Type (ii) this has even length because $I$ and $J$ have the same length, but in Type (iii) the length is odd because $j_{d}$ is missing from $J$.

Types (iv)-(vii) are the types built from block cycle 4-tuples. Among these, Type (vii) is a direct sum of two block cycle 4-tuples while (iv)-(vi) 
each consist of a single block cycle 4-tuple. Among Types (iv)-(vi), the label sequences of $f(p)$ have even length in Types (iv) and (vi) because $I$ and $J$ have the same length, and odd length in Type (v) because $j_{d}$ is missing from $J$. Thus, it remains to distinguish between Types (iv) and (vi), the most subtle of the distinctions.

Recall that both label sequences $I$ and $J$ of $p$ have the same length, say $d$, in each of situations (iv) and (vi). The label sequences of $f(p)$ are

$$
\begin{array}{ll}
\{I, \mu(J)\} \text { and }\{J, \mu(I)\} & \text { in Type (iv) } \\
\{J, \mu(I)\} \text { and } \nu(\{I, \mu(J)\}) & \text { in Type (vi). }
\end{array}
$$

After writing $H=\{I, \mu(J)\}$ and $K=\mu(H)$ these sequences become

$$
\begin{array}{ll}
H \text { and } \mu(H) & \text { in Type (iv) } \\
K \text { and } \nu \mu(K) & \text { in Type (vi). }
\end{array}
$$

Note that $H$ and $K$ both have even length $e=2 d$. Therefore, by Lemma 8.13, a self-conjugate block cycle 4-tuple with one of the pairs of label sequences displayed in (8.18.2) can never be isomorphic to one with the other pair of label sequences. This shows that no pair of Type (iv) can be isomorphic to a pair of Type (vi), and completes our proof of the uniqueness of types.

We now proceed to the remaining uniqueness properties of each individual type. In view of the uniqueness of types, we may assume, for the rest of this proof that $p$ and $q$ are of the same type (i)-(vii).

Types (i)-(iii). Here we frequently use, without explicitly mentioning it, that two deleted cycle 4-tuples are isomorphic if an only if their pairs of label sequences are the same [Theorem 7.3].

Type (i): Nonreduced. Here $f(p) \cong g \oplus \bar{g}$ where $g$ is the deleted cycle 4tuple with label sequences $I$ and $J$, and hence $\bar{g}$ is isomorphic to the deleted cycle 4-tuple with label sequences $\mu(J)$ and $\mu(I)$ [Lemma 8.8]. Similarly $f(q) \cong h \oplus \bar{h}$ where the label sequences of $h$ are $I^{\prime}$ and $J^{\prime}$, and $\bar{h}$ is isomorphic to the deleted cycle 4 -tuple with label sequences $\mu\left(J^{\prime}\right)$ and $\mu\left(I^{\prime}\right)$. If $h \cong g$ then $I^{\prime}=I$ and $J^{\prime}=J$, the first possibility stated in part (i) of the theorem. Otherwise, since $g, \bar{g}, h, \bar{h}$ are all indecomposable 4-tuples and the KrullSchmidt Theorem holds for 4-tuples, we have $h \cong \bar{g}$; and therefore $I^{\prime}=\mu(J)$ and $J^{\prime}=\mu(I)$, the second possibility stated in part (i) of the theorem.

Conversely, if $p$ and $q$ are nonreduced pairs with $I^{\prime}=I$ and $J^{\prime}=J-$ or with $I^{\prime}=\mu(J)$ and $J^{\prime}=\mu(I)$ - then reversing the above argument easily yields $p \cong q$.

Types (ii) and (iii): Bottom-reduced and top-reduced, respectively. In both types $f(p)$ is a single deleted cycle 4-tuple, with label sequences $\{I, \mu(J)\}$ and $\{J, \mu(I)\}$. A similar statement holds for $f(q), I^{\prime}$ and $J^{\prime}$.

Suppose $p \cong q$, and hence $f(p) \cong f(q)$. Since isomorphic deleted cycle 4 -tuples have the same pairs of label sequences, we have $\{I, \mu(J)\}=$ $\left\{I^{\prime}, \mu\left(J^{\prime}\right)\right\}$, which is equivalent to $I=I^{\prime}$ and $J=J^{\prime}$, as desired. 
Conversely, since $f(p)$ and $f(q)$ are each a single deleted cycle 4-tuple, if $I^{\prime}=I$ and $J^{\prime}=J$, then $f(p) \cong f(q)$ and hence $p \cong q$.

We now turn to the types involving block cycle 4-tuples. Let $F$ and $F^{\prime}$ be such 4-tuples, where $F$ has label sequences $H$ and $K$, and $F^{\prime}$ has label sequences $H^{\prime}$ and $K^{\prime}$. Recall [Theorem 7.3] that $F \cong F^{\prime}$ if and only both 4 -tuples have similar blocking matrices and, for some $t$,

$$
\nu^{t}(H)=H^{\prime} \quad \text { and } \quad \nu^{t}(K)=K^{\prime} .
$$

Type (vii): Cycle pairs. Here, $f(p) \cong g \oplus \bar{g}$, where $g$ is the block cycle 4-tuple with label sequences $I$ and $J$ and blocking matrix $U$. Therefore $\bar{g}$ is isomorphic to the block cycle 4-tuple with label sequences $\mu(J)$ and $\mu(I)$ and blocking matrix $\bar{U}^{-1}$ [Lemma 8.12]. Similarly, $f(q) \cong h \oplus \bar{h}$ where $h$ has label sequences $I^{\prime}$ and $J^{\prime}$ and blocking matrix $V$, and $\bar{h}$ has label sequences $J^{\prime}$ and $I^{\prime}$ and blocking matrix $\bar{V}^{-1}$

As in the the analysis of Type (i) above, we have either $h \cong g$ or $h \cong \bar{g}$. In the situation $h \cong g$, we have $I^{\prime}=\nu^{t}(I)$ and $J^{\prime}=\nu^{t}(J)$ (for some $t$ ) and $V$ is similar to $U$ (see (8.18.3)). Similarly, in the situation $h \cong \bar{g}$ we have $I^{\prime}=\nu^{t} \mu(J)$ and $J^{\prime}=\nu^{t} \mu(I)$ (for some $t$ ) and $V$ is similar to $\bar{U}^{-1}$. As in Type (i), the converse follows easily by reversing the argument.

Types (iv)-(vi): Bottom-bottom-reduced, bottom-top-reduced, and toptop-reduced, respectively. Here $f(p)$ is isomorphic to a single block cycle 4-tuple. Its label sequences are

$$
\begin{aligned}
& \{I, \mu(J)\} \quad \text { and } \quad\{J, \mu(I)\} \\
& \{J, \mu(I)\} \text { and } \nu(\{I, \mu(J)\})
\end{aligned}
$$

and the blocking matrix is $U \bar{U}^{-1}$ in all three types. Similar remarks apply to $f(q)$.

Suppose that $p \cong q$. Then $f(p) \cong f(q)$ and hence their blocking matrices $V \bar{V}^{-1}$ and $U \bar{U}^{-1}$ are similar, as desired. Let $f(p)$ have label sequences $H$ and $K$; and let $f(q)$ have label sequences $H^{\prime}$ and $K^{\prime}$. Then (8.18.3) holds. Let $d$ be the number of terms in the sequence $I$. We now consider each of these three types separately.

Type (v): Bottom-top reduced. Here, the number of terms in $(\{I, \mu(J)\}$ is $2 d-1$, because $j_{d}$ is missing in $J$. In view of (8.18.4), relations (8.18.3) become

$$
\nu^{t}(\{I, \mu(J)\})=\left\{I^{\prime}, \mu\left(J^{\prime}\right)\right\} \quad \text { and } \quad \nu^{t}(\{J, \mu(I)\})=\left\{J^{\prime}, \mu\left(I^{\prime}\right)\right\} .
$$

The second equation in (8.18.5) can be rewritten as $\nu^{t} \mu(\{I, \mu(J)\})=\mu\left(\left\{I^{\prime}\right.\right.$, $\left.\left.\mu\left(J^{\prime}\right)\right\}\right)$. By (8.6.1), we can substitute $\nu^{t} \mu=\mu \nu^{-t}$, so after canceling $\mu$, we obtain the equation $\nu^{-t}(\{I, \mu(J)\})=\left\{I^{\prime}, \mu\left(J^{\prime}\right)\right\}$. Combining this with the first equation in (8.18.5) then yields $\nu^{-t}(\{I, \mu(J)\})=\nu^{t}(\{I, \mu(J)\})$, and hence $\nu^{2 t}(\{I, \mu(J)\})=\{I, \mu(J)\}$. By the "repetition-free" condition 
(8.10.2)(ii), the permutation $\nu^{2 t}$ must act as the identity on the sequence $\{I, \mu(J)\}$, from which it follows that $2 d-1$ (the length of this sequence) divides $2 t$. But then $2 d-1$ divides $t$, so that $\nu^{t}$ is the identity permutation in (8.18.5). This completes the proof that $q$ satisfies the conditions for Type $(\mathrm{v})$.

Type (iv): Bottom-bottom reduced. Here $I$ and $J$ have the same length $d$, and therefore the concatenated sequence $\{I, \mu(J)\}$ has length $2 d$. The same argument as for Type (v) above still applies, with one twist: When we reach the relation $\nu^{2 t}(\{I, \mu(J)\})=\{I, \mu(J)\}$, the "repetition-free" condition implies that $2 d$ (rather than $2 d-1$ ) divides $2 t$, and hence $d$ divides $t$, say $t=x d$. This yields the two possibilities for Type (iv) as follows. If $x$ is even, then $2 d$ divides $t$, in which case $\nu^{t}$ is the identity permutation in (8.18.5), and therefore $I=I^{\prime}$ and $J=J^{\prime}$.

Otherwise $x$ is odd, say $x=2 y+1$ and hence $\nu^{t}=\nu^{(2 y+1) d}=\nu^{d}$. Then the second equation of (8.18.5) yields $\mu(I)=J^{\prime}$ and $J=\mu\left(I^{\prime}\right)$. The second of these is equivalent to $I^{\prime}=\mu(J)$. This completes the proof that $q$ satisfies the conditions for Type (iv).

Type (vi): Top-top reduced. This is similar to Type (iv). $I$ and $J$ have the same length $d$, and therefore the two concatenated sequences in the second row of (8.18.4) have length $2 d$. Substituting these concatenated sequences into (8.18.3), and then canceling $\nu$ from the second of equation, again yields the pair of equations (8.18.5). As in Type (v) this implies $\nu^{2 t}(\{I, \mu(J)\})=\{I, \mu(J)\}$. Since the concatenated sequences have length $2 d$ we finish exactly as in Type (iv), reaching the same conclusion as in Type (iv), as desired.

This completes the proof that $q$ satisfies the conditions for Type (vi), and hence for all types.

The converse parts of (iv)-(vi) are easily verified by using the fact that isomorphism of block cycle 4-tuples is implied by the equations in (8.18.3), for some $t$, together with similarity of blocking matrices.

We conclude this section by noting the structure of those matrix pairs that fail the "nonsymmetry conditions" in (8.7.2)(ii) or (8.10.5)(i).

Proposition 8.19. Let $p$ be a matrix pair. Suppose that either:

(i) $p$ would be a nonreduced pair except that its pair of label sequences has the form $I$ and $\mu(I)$; or

(ii) $p$ would be a cycle pair except that its pair of label sequences has the form $I$ and $\nu^{t} \mu(I)$ for some cycle $\nu^{t}$, and its blocking matrix $L$ is similar to $\bar{L}^{-1}$.

Then $p \cong p^{\prime} \oplus p^{\prime}$ for some indecomposable matrix pair $p^{\prime}$.

Proof. (ii) By (8.14.5) we have $f(p) \cong g \oplus \bar{g}$ where $g$ is the block cycle pair whose blocking matrix is $L$ and whose label sequences are the same 
as the pair used to define $p$, that is, $I$ and $\nu^{t} \mu(I)$. By Lemma 8.12, $g$ is self-conjugate, and therefore $f(p) \cong g \oplus g$. Since $g \cong \bar{g}$, the 4-tuple $g$ is a package [Theorem 8.15]; say $g \cong f\left(p^{\prime}\right)$. Then $f(p) \cong f\left(p^{\prime} \oplus p^{\prime}\right)$ and therefore $p \cong p^{\prime} \oplus p^{\prime}$ [Theorem 8.3]. Moreover, $g \cong f\left(p^{\prime}\right)$ is indecomposable since it is a block cycle 4-tuple. Therefore $p^{\prime}$ is also indecomposable.

(i) This is essentially the same as the proof of (ii), except that one uses the deleted cycle analogues, (8.9.6) and Lemma 8.8, of the corresponding block cycle results in that proof.

\section{Proofs: Indecomposable modules, unsplit case.}

In this section $(\Lambda, \mathfrak{m}, k)$ denotes an unsplit Dedekind-like ring with normalization $(\Gamma, \mathfrak{m}, F)$. We prove the structure theorems about indecomposable $\Lambda$-modules stated in $\S 2$.

9.1. Connection with matrices (Brief review). Recall that a "matrix setup" $\mathcal{X}$ is a finite external direct-sum decomposition $X=\oplus_{\nu} \Gamma / \mathfrak{m}^{t_{\nu}}(1 \leq$ $t_{\nu} \leq \infty$, where $\left.\mathfrak{m}^{\infty}=0\right)$, the " $\Gamma$-module associated with $\mathcal{X}$ " [Notation 5.6]. Corresponding to certain pairs $(A, B)$ of matrices over $F$, with $A$ invertible, we define three finitely generated $\Lambda$-modules,

$$
\begin{gathered}
M=M(A, B)=S(A) / K(B) \quad \text { where } \\
S(A)=\left\{\boldsymbol{x} \in X \mid \rho(\boldsymbol{x}) \in k^{(n)} \cdot A\right\} \quad \text { and } \\
K(B)=k^{(q)} \cdot B .
\end{gathered}
$$

The matrix $A$ has one column for each uniserial summand $\Gamma / \mathfrak{m}^{t}$ of $X$, and $B$ has one column for each of these uniserial summands that has finite length. Thus each column of $A$ has an associated uniserial summand of $X$ and an associated column of $B$. We attach a "length label" to each column of $A$ and of $B$, namely the length of its associated uniserial summand of $X$ [Definition 5.8]. We always view $B$ as being placed under $A$, with each column of $B$ written under the corresponding column of $A$ and no column of $B$ under each column of $A$ whose corresponding summand of $X$ has infinite length.

Given matrix setups $\mathcal{X}$ and $\mathcal{X}^{\prime}$ and associated matrix pairs $(A, B)$ and $\left(A^{\prime}, B^{\prime}\right)$, we defined $(A, B) \cong\left(A^{\prime}, B^{\prime}\right)$ to mean that either matrix pair can be obtained from the other by means of two types of matrix operations that we call "display operations" [Definition 5.9] and " $k-F$ sweeping-similarity operations" [Theorem 5.11]. Then we proved that $(A, B) \cong\left(A^{\prime}, B^{\prime}\right)$ if and only if $M(A, B) \cong M\left(A^{\prime}, B^{\prime}\right)$ [Theorem 5.15].

Since the $\Lambda$-module corresponding to the direct sum of two matrix pairs $(A, B)$ (computed with respect to the direct sum of their associated matrix setups) is obviously isomorphic to the direct sum of the associated modules $M(A, B)$, and since every finitely generated $\Lambda$-module is isomorphic to some 
$M(A, B)$ [Theorem 5.10], we conclude that $M(A, B)$ is an indecomposable $\Lambda$-module if and only if $(A, B)$ is an indecomposable matrix pair.

Fix an element $\varepsilon \in F-k$, and note that we then have $F=k[\varepsilon]$.

9.2. Proof of Theorem 2.7 (Indecomposable modules come from standard diagrams and conversely). In Definitions 8.7 and 8.10 we defined seven canonical forms of matrix pairs $(A, B)$. Each of these canonical forms has the same name as one of our seven types of standard diagrams [Definitions 2.4 and 2.6].

The supplementary statements at the end of Theorems 8.9 and 8.14 state that $M(A, B)$ is indecomposable whenever $(A, B)$ is one of these pairs. Conversely, Corollary 8.17 states that every indecomposable finitely generated $\Lambda$-module is isomorphic to one of this form.

Thus is now suffices to prove:

(9.2.1) Let $(A, B)$ be a matrix pair of one of the seven canonical types. Then there is an associated standard diagram $\mathcal{D}$ (described below), whose type has the same name as the type of $(A, B)$ and such that $M(A, B) \cong M(\mathcal{D})$. Moreover, the set of diagrams that arise in this way is precisely the set of standard diagrams.

Construct the diagram $\mathcal{D}$ associated with a canonical pair $(A, B)$ as follows. This pair is associated with a matrix setup $\mathcal{X}$ whose associated $\Gamma$ modules is decomposed as displayed in (2.2.1). We call the integer $m$ in this decomposition the block size of $\mathcal{X}$ and of $(A, B)$. Thus the pairs arising from deleted cycle 4-tuples [see Definition 8.7] all have block size 1. And the block size of any pair arising from a block cycle 4-tuple [Definition 8.10] equals the number of rows and columns in the blocking matrix of the pair.

First construct a diagram $\mathcal{D}_{0}$ (not a standard diagram!), which consists of one vertical bar for each block of $m$ uniserial summands of $X$, with the top of each bar labeled by the common length of the uniserial summands in that block. When we refer to column blocks of $A$ or $B$, we always mean the $m$-column blocks associated with blocks of uniserial summands of $X$. Thus every basic tile that occurs in $A$ or $B$ has two associated vertical bars in $\mathcal{D}_{0}$, corresponding to the two column blocks that pass through it. Similarly, every "exceptional tile" (that is, nonzero $m \times m$ block $U, U^{-1}$, or $I_{m}$, that is not part of a basic tile) of $A$ or $B$ has exactly one associated vertical bar in $\mathcal{D}_{0}$.

Form $\mathcal{D}$ by attaching edges to $\mathcal{D}_{0}$ according to the following rules.

(9.2.2) (i) For each basic tile that occurs in $A$, connect its two corresponding vertical bars with a top-gluing edge. If $U$ occurs in this basic tile (and hence in the first of the two column blocks of the tile), label the lefthand end of the edge with $U$. 
(ii) For each basic tile that occurs in $B$, connect the two corresponding vertical bars with a bottom-gluing edge.

(iii) For each exceptional tile that occurs in $A$ attach a top-reduction edge to the corresponding vertical bar. If the tile consists of the blocking matrix $U$, label the top-reduction edge with $U$.

(iv) For each exceptional tile that occurs in $B$, attach a bottom-reduction edge to the corresponding vertical bar. If the tile consists of the inverse of the blocking matrix, label the bottom-reduction edge with $U^{-1}$.

It is immediate that $\mathcal{D}$ is the standard diagram with the same name, label sequences and (when relevant) blocking matrix as the canonical form $(A, B)$.

Next we show that $S(A)=S(\mathcal{D})$. Choose any basic tile $T$ that occurs in $A$. This tile interacts with precisely two uniserial summand-blocks of $X$, namely those corresponding to the column blocks containing $T$, and $T$ is the only tile contained in $A$ that interacts with either of these summand-blocks. Let the length labels of the first and second column blocks of $T$ be $i$ and $j$ respectively, and denote the corresponding direct sum of uniserial summands of $X$ by $X_{i}$ and $X_{j}$ respectively (ignoring the slight abuse of notation that results when $X_{i}$ and $X_{j}$ have the same length; that is, $i=j$ and $X_{i} \neq X_{j}$ ). Thus $X_{i}=\left(\Gamma / \mathfrak{m}^{i}\right)^{(m)}$ and $X_{j}=\left(\Gamma / \mathfrak{m}^{j}\right)^{(m)}$. If $\boldsymbol{x} \in X_{i}$ and $\boldsymbol{y} \in X_{j}$ and $(\boldsymbol{x}, \boldsymbol{y}) \in S(A)$, then Formula (9.1.1) implies that $\rho(\boldsymbol{x}, \boldsymbol{y}) \in\left(k^{(m)} \oplus k^{(m)}\right) T$. Writing this out, using the form of a basic tile given in (8.4.1), yields

$$
(\rho(\boldsymbol{x}), \rho(\boldsymbol{y}))=((\boldsymbol{a}+\boldsymbol{b} \varepsilon) U, \boldsymbol{a}+\boldsymbol{b} \bar{\varepsilon}) \quad\left(\boldsymbol{a}, \boldsymbol{b} \in k^{(m)}\right)
$$

where, to save space, we use $U$ to denote either the blocking matrix or $I$, whichever is appropriate.

Writing $\boldsymbol{\alpha}=\boldsymbol{a}+\boldsymbol{b} \varepsilon$ we see that the ordered pair in (9.2.3) equals $(\boldsymbol{\alpha} U, \overline{\boldsymbol{\alpha}})$ where $\boldsymbol{\alpha}$ is an arbitrary element of $F^{(m)}$. Therefore $\rho(\boldsymbol{x})=\bar{\rho}(\boldsymbol{y}) U$; in other words, $X_{i}$ and $X_{j}$ are top-glued as in (2.2.4).

On the other hand, consider any exceptional tile that occurs in $A$. This block equals $U$ or $I$; we write $U$ in either case. This tile interacts with the uniserial summand $X_{i}$ of $X$ that corresponds to the column block containing the tile, and is the only tile in $A$ that interacts with $X_{i}$. Take $\boldsymbol{x} \in X_{i}$. If $\boldsymbol{x} \in S(A)$ then (9.1.1) implies that $\rho(\boldsymbol{x}) \in k^{(m)} U$. Thus $X_{i}$ is top-reduced in the sense of (2.2.7). This completes the proof that $S(A)=S(\mathcal{D})$.

$K(B)=K(\mathcal{D})$. The analysis here is very similar to the analysis of $S(A)$ except that the basic and exceptional tiles (whichever occur) result in bottom gluing and bottom reduction, respectively.

Thus we now have $M(A, B)=M(\mathcal{D})$. It is clear that all standard diagrams occur in this way.

9.3. Proof of Theorem 2.8 (Uniqueness of standard diagrams). See Theorem 8.18. 
9.4. Proof of Proposition 2.9 (Moving $U$ ). Statement (i) was proved in (2.2.12). Let $M=M(\mathcal{D})$, where $\mathcal{D}$ is a standard diagram, except that the blocking matrix $U$ labels an arbitrary gluing or reduction edge (to be specified).

Statement (ii). We want to show that moving $U$ to the opposite end of its attached vertical bar and then changing it to $U^{-1}$ does not change the isomorphism class of $M$. If this move can be done, then it can also be done in reverse, so there is no loss of generality in assuming that we start at the top of a vertical bar. We need to consider seven situations separately (see (9.4.1)). In each situation we wish to move $U$ from position 1 to position 2 , replacing it by $U^{-1}$. In most situations we place a matrix $U$ at position 3 and then compare the effects of moving it to position 1 and position 2 , and determine how $U$ must then be changed to preserve the isomorphism class of $M$. We consider the seven situations in the order in which they are displayed, doing the first in more detail than the others.
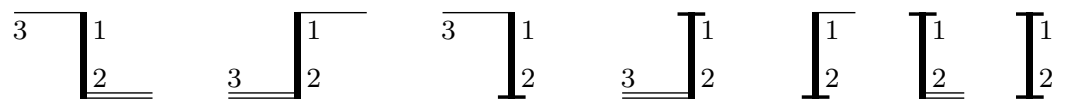

Let $\mathcal{D}$ be the diagram before the blocking matrix is moved. Thus $\mathcal{D}$ is a standard diagram except that $U$ is in a position that we shall specify. In the notation of Subsection 9.1 , we have $M=S(A) / K(B)$ for the matrix pair $(A, B)$ over $F$, where $(A, B)$ is in one of our standard canonical forms - the form with the same name as $\mathcal{D}$ - except that the blocking matrix is located in a basic tile to be specified, instead of the standard place.

Our proof makes use of the matrix 4-tuple $(A, \bar{A}, B, \bar{B})$ corresponding to the given matrix pair. Recall that $(A, B) \cong\left(A^{\prime}, B^{\prime}\right)$ if and only if $(A, \bar{A}, B, \bar{B}) \cong\left(A^{\prime}, \overline{A^{\prime}}, B^{\prime}, \overline{B^{\prime}}\right)$ [Theorem 8.3]. We always assume that $M(A, B) \cong M(\mathcal{D})$ before the blocking matrix is moved, and then prove that the isomorphism continues to hold afterwards. Our proofs make use of the fact that sweeping-similarity operations do not change the isomorphism class of $(A, \bar{A}, B, \bar{B})$ [Theorem 6.7]. But recall that we use $F-F$ sweepingsimilarity instead of $k$ - $k$-similarity since our matrices have entries in $F$.

Top-gluing edge at left (of vertical bar) to bottom-gluing edge. This is the first situation displayed in (9.4.1). We assume that $U$ is placed in position 3 . By statement (i) we can move $U$ to position 1, changing it to $\bar{U}^{-1}$. Therefore it suffices to prove that we can move $U$ from position 3 to position 2 , changing it to $\bar{U}$.

The matrix 4-tuple in (9.4.2) shows the portion of $(A, \bar{A}, B, \bar{B})$ that we wish to alter: We wish to move $\mathrm{U}$ from the two top tiles to the two bottom tiles and replace it with $\bar{U}$. 


$$
\begin{aligned}
& {\left[\begin{array}{ccc}
U & I & \\
U \varepsilon & I \bar{\varepsilon} &
\end{array}\right]\left[\begin{array}{ccc}
\bar{U} & I & \\
\bar{U} \bar{\varepsilon} & I \varepsilon &
\end{array}\right]} \\
& {\left[\begin{array}{ccc} 
& I & I \\
& I \varepsilon & I \bar{\varepsilon}
\end{array}\right]\left[\begin{array}{ccc} 
& I & I \\
& I \bar{\varepsilon} & I \varepsilon
\end{array}\right] .}
\end{aligned}
$$

The first step is to reduce the basic tiles, using Lemma 8.4 twice. This changes them to the form shown in the 4-tuple in (9.4.3).

$$
\begin{aligned}
& {\left[\begin{array}{lll}
U & 0 & \\
0 & I &
\end{array}\right]\left[\begin{array}{ccc}
0 & I & \\
\bar{U} & 0 &
\end{array}\right]} \\
& {\left[\begin{array}{lll} 
& I & 0 \\
& 0 & I
\end{array}\right]\left[\begin{array}{lll} 
& 0 & I \\
& I & 0
\end{array}\right] .}
\end{aligned}
$$

Next, left multiplication of the first row-block of the upper two matrices by $U^{-1}$ and the second row-block by $\bar{U}^{-1}$ yields the form shown in (9.4.4).

$$
\begin{aligned}
& {\left[\begin{array}{ccc}
I & 0 & \\
0 & \bar{U}^{-1} &
\end{array}\right]\left[\begin{array}{ccc}
0 & U^{-1} & \\
I & 0 &
\end{array}\right]} \\
& {\left[\begin{array}{ccc} 
& I & 0 \\
& 0 & I
\end{array}\right]\left[\begin{array}{ccc} 
& 0 & I \\
& I & 0
\end{array}\right] .}
\end{aligned}
$$

Then, right multiplication of the second column-block of the first upperlower pair by $\bar{U}$ and the second column-block of the second upper-lower pair by $U$ yields the form shown in (9.4.5).

$$
\begin{aligned}
& {\left[\begin{array}{lll}
I & 0 & \\
0 & I &
\end{array}\right]\left[\begin{array}{lll}
0 & I & \\
I & 0 &
\end{array}\right]} \\
& {\left[\begin{array}{ccc} 
& \bar{U} & 0 \\
& 0 & I
\end{array}\right]\left[\begin{array}{ccc} 
& 0 & I \\
& U & 0
\end{array}\right] .}
\end{aligned}
$$

Finally, note that this matrix 4-tuple in (9.4.5) can also be obtained by starting with the 4-tuple in (9.4.6) and applying Lemma 8.4 to each of the two pairs of basic tiles:

$$
\begin{aligned}
& {\left[\begin{array}{lll}
I & I & \\
I \varepsilon & I \bar{\varepsilon} &
\end{array}\right]\left[\begin{array}{ccc}
I & I & \\
I \bar{\varepsilon} & I \varepsilon &
\end{array}\right]} \\
& {\left[\begin{array}{ccc} 
& \bar{U} & I \\
& \bar{U} \varepsilon & I \bar{\varepsilon}
\end{array}\right]\left[\begin{array}{ccc} 
& U & I \\
& U \bar{\varepsilon} & I \varepsilon
\end{array}\right] .}
\end{aligned}
$$

This completes the proof that we can move $U$ from position 3 to $\bar{U}$ in position 2 .

Top-gluing edge at right (of vertical bar) to bottom-gluing edge. This is done similarly. 
Top-gluing edge at left (of vertical bar) to bottom-reduction edge. We want to change the first 4-tuple displayed in (9.4.7) to the second. The procedure is the same as the first situation we considered.

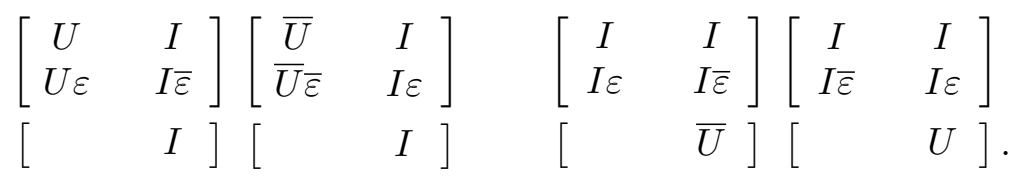

Top-reduction edge to bottom-gluing edge at left (of vertical bar). Interchange the roles of $A$ and $B$ in the previous case.

Top-gluing edge at right (of vertical bar) to bottom-reduction edge. Here we ignore position 3 , and move $U$ directly from position 1 to position 2 . Moreover, it is easier to ignore the 4-tuple and work directly with $(A, B)$, transforming the first matrix pair in (9.4.8) to the second by means of the $k-F$ sweeping-similarity operations of Theorem 5.11. In fact, we need only right-multiply the first column block of the first matrix pair by $U^{-1}$ in order to obtain the second matrix pair.

$$
\begin{array}{lll}
{\left[\begin{array}{cc}
U & I \\
U \varepsilon & I \bar{\varepsilon}
\end{array}\right]} & {\left[\begin{array}{cc}
I & I \\
I \varepsilon & I \bar{\varepsilon}
\end{array}\right]} \\
{\left[\begin{array}{ll}
I & ]
\end{array}\right.} & {\left[U^{-1}\right.} & ] .
\end{array}
$$

Top-reduction edge to bottom-gluing edge at right (of vertical bar). Reverse the roles of $A$ and $B$ in the previous case.

Top-reduction edge to bottom-reduction edge. This again is the same, but replace the two basic tiles ( $A$-matrices) in (9.4.8) by the exceptional tiles $U$ and $I$, respectively.

Supplementary statement. Now suppose that $\mathcal{D}$ is one of the standard diagrams with a pair of reduction edges. Then the similarity invariant of $\mathcal{D}$ is the similarity class of $U \bar{U}^{-1}$ rather than of $U$ itself [Theorem 2.8]. Suppose that $U$ is attached to some top gluing edge. Then replacing $U$ by $\bar{U}^{-1}$, and not moving it, leaves the similarity invariant of $\mathcal{D}$ unchanged. Now, by (i), moving $\bar{U}^{-1}$ to the other end of the gluing edge replaces it by $U$ again. Thus, moving $U$ from the top of one vertical bar to the top of an adjacent vertical bar does not change the isomorphism class of $M(\mathcal{D})$. After doing this, we can move $U$ to the bottom of that bar, replacing it by $U^{-1}$. If that bar has an attached gluing edge, the same reasoning as before allows us to move $U^{-1}$ to the other end of that edge without changing the isomorphism class of $M(\mathcal{D})$. Since all standard diagrams are connected diagrams, a combination of these moves enable us to move $U$ from top to top (or, similarly, bottom to bottom) of any pair of vertical bars.

Proposition 9.5. Suppose that one of the following holds.

(i) $\mathcal{D}=\mathcal{D}_{\mathrm{Nrd}}$, except that Condition (2.4.2)(iii) fails (that is, $J=\mu(I)$ holds); or 
(ii) $\mathcal{D}=\mathcal{D}_{\mathrm{Cy}}$, except that Condition (2.6.4)(ii) fails (that is, we have both $U \sim \bar{U}^{-1}$ and $J=\nu^{t} \mu(I)$ for some cycle $\left.\nu^{t}\right)$.

Then $M(\mathcal{D})$ is the direct sum of two isomorphic indecomposable $\Lambda$-modules.

Proof. As at the end of Subsection 9.2, we have $M(A, B)=M(\mathcal{D})$ for an appropriate $(A, B)$. Therefore this proposition is a module-theoretic translation of Proposition 8.19.

9.6. Proof of Theorem 2.11 ( $\Lambda$-homomorphisms versus $\Gamma$-homomorphisms). Recall that a "separated $\Lambda$-module" is a $\Lambda$-submodule of some $\Gamma$ module, and that a "separated cover", the fundamental structure on which this paper is built, is defined in Definition 4.6.

Now let $\phi: S(\mathcal{D}) \rightarrow M(\mathcal{D})$ be the natural homomorphism. We claim that $\phi$ is a separated cover. We already observed that $M(\mathcal{D}) \cong M(A, B)=$ $S(A) / K(B)$ for a suitable matrix pair $(A, B)[(9.1 .1)]$, and, at the end of Subsection 9.2, we showed that $S(A)=S(\mathcal{D})$ and $K(B)=K(\mathcal{D})$. Therefore the claim follows from Theorem 5.10(ii). Similarly, $\phi^{\prime}: S\left(\mathcal{D}^{\prime}\right) \rightarrow M\left(\mathcal{D}^{\prime}\right)$ is a separated cover.

Our main "almost functorial property" of separated covers [Theorem 4.12] states that any homomorphism $f$ of finitely generated $\Lambda$-modules can be lifted to a $\Lambda$-homomorphism $f^{*}$ of their separated covers, and this lifting preserves monomorphisms and surjections. Thus it now suffices to further extend $f^{*} S\left(\mathcal{D}^{\prime}\right) \rightarrow S(\mathcal{D})$ to a $\Gamma$-homomorphism $f^{* *}: X\left(\mathcal{D}^{\prime}\right) \rightarrow X(\mathcal{D})$.

First note that $X(\mathcal{D})=\Gamma \cdot S(\mathcal{D})$ and $X\left(\mathcal{D}^{\prime}\right)=\Gamma \cdot S\left(\mathcal{D}^{\prime}\right)$, by the second equation in (9.1.1), because the matrices $A$ and $A^{\prime}$ are invertible. Now $\Gamma \cdot S(\mathcal{D}) \cong \Gamma \otimes_{\Lambda} S(\mathcal{D})$ and $\Gamma \cdot S\left(\mathcal{D}^{\prime}\right) \cong \Gamma \otimes_{\Lambda} S\left(\mathcal{D}^{\prime}\right)$, by Lemma 5.2, so that $f^{* *}=1 \otimes f^{*}$ is the desired extension of $f^{*}$.

Remark 9.7 (on repetition-freeness). When the condition on repetitionfreeness (2.6.4)(iv) fails for cycle diagrams, it is easy to see that a larger block size can be used, with a correspondingly different blocking matrix. The new, larger blocking matrix can fail to be indecomposable under similarity, in which case $M(\mathcal{D})$ decomposes.

When the repetition-freeness condition (2.6.3)(iii) fails for one of the other three diagrams that occur with arbitrary block size, a similar (but slightly more complicated) thing happens. Let $p$ be the matrix pair for which the condition fails. Then, in the associated 4-tuple $f(p)$, a larger block size can be used. Let its (larger) blocking matrix be $L$. Our desire for a canonical form then allows us to disregard the smaller block size. Changing to the larger blocking matrix $L$ can also change the type of diagram that we are dealing with. Moreover, the new, larger blocking matrix might be decomposable under similarity, which easily shows that $f(p)$ decomposes. If this decomposition of $f(p)$ contains a pair of mutually conjugate summands or a single self-conjugate summand, and contains additional terms, then $f(p)$ is 
a direct sum of two packages, and hence $p$ decomposes. In this case, $M(p)$ decomposes as well.

\section{Proofs: Indecomposable modules, split case.}

In this section $(\Lambda, \mathfrak{m}, k)$ denotes a strictly split Dedekind-like ring with normalization $\Gamma$. The proofs in this section are minor modifications of the corresponding similar — but not identical — results in the unsplit case. The purpose of the truncated proofs that follow is to give a directory to the needed earlier results in this paper.

10.1. Connection with matrices (Brief review). Let $M$ be any finitely generated $\Lambda$-module. Then $M \cong M\left(A_{1}, A_{2}, B_{1}, B_{2}\right)$ where $\left(A_{1}, A_{2}, B_{1}, B_{2}\right)$ is a matrix 4-tuple (over $k$ ) associated with some matrix setup $\mathcal{X}$ [Notation 6.3 - Theorem 6.6]. Moreover, two such modules are isomorphic if and only if their corresponding matrix 4-tuples can be obtained from each other by means of $k$ - $k$ sweeping-similarity operations and display operations [Theorem 6.10].

Now suppose that $M$ is indecomposable. Then, after suitable $k$ - $k$ sweeping-similarity operations and display operations, $\left(A_{1}, A_{2}, B_{1}, B_{2}\right)$ becomes either a deleted cycle 4-tuple or a block cycle 4-tuple [Theorem 7.4], and each 4-tuple of either of these types yields an indecomposable $M$ [Theorem 7.3].

10.2. Proof of Theorem 3.5 (Indecomposable modules versus standard diagrams). The first step is to show that each deleted cycle or block cycle 4-tuple yields, respectively, a module $M\left(\mathcal{D}_{\mathrm{DCy}}\right)$ or $M\left(\mathcal{D}_{\mathrm{BCy}}\right)$, and this is an easy consequence of the definitions of these two types of 4-tuples [Definitions 7.2] and diagrams [Definitions 3.3]. Thus it now suffices to prove the uniqueness assertions of Theorem 3.5, and these follow from Theorem 7.3.

10.3. Proof of Proposition 3.6 (Moving $U$ ). This proof refers to the block cycle 4-tuple displayed in diagram (7.2.4), where the blocking matrix is called $L$ instead of $U$. The matrix operations we use are among the $k-k$ sweeping-similarity operations listed in Theorem 6.7.

We can simultaneously left multiply the first $m$ rows of $A_{1}$ and $B_{1}$ by $L^{-1}$, thus replacing the $L$ in the upper left corner of $A_{1}$ by $I$ and replacing the $I$ in the upper left corner of $A_{2}$ by $L^{-1}$. Therefore, we have moved $L$ from the left side of a top-gluing edge in $\mathcal{D}$ to the right side of that edge, replacing $L$ by $L^{-1}$, as desired.

Once $L^{-1}$ is in this new position, we simultaneously right multiply the $j_{1}$-labeled columns of $A_{2}$ and $B_{2}$ by $L$, thus replacing $L^{-1}$ by $I$ and replacing the $I$ below it (in $B_{2}$ ) by $L$. Therefore, we have moved $L^{-1}$ from the top 
of the $j_{1}$-labeled bar in $\mathcal{D}$ to the bottom of that bar, replacing it by $L$, as desired. Note that $L$ is now attached to the bottom-gluing edge attached to the $j_{1}$-labeled vertical bar.

Analogous simultaneous row operations in $B_{1}$ and $B_{2}$ now move $L$ from one end of its attached bottom-gluing edge to the other, replacing it by $L^{-1}$. Continuing in the fashion around the entire cycle completes the proof of the proposition.

10.4. Proof of Theorem 3.8 ( $\Lambda$-homomorphisms versus $\Gamma$-homomorphisms). We have $M=S / K$ where $S=S(\mathcal{D})$ and $K=K(\mathcal{D})$, as in (3.2.9). If we can show that the natural homomorphism $S \rightarrow S / K$ is a separated cover of $S / K \cong M$, then we can just repeat the proof, in Subsection 9.6, of the corresponding theorem in the unsplit case. For this, see Theorem 6.6.

\section{Klein rings and homomorphic images of Dedekind-like rings.}

In this section we complete our commutative noetherian tame-wild theorem (complete local case) by describing all indecomposable finitely generated modules over Klein rings (defined below). We also show how to use our description of indecomposable modules over Dedekind-like rings [§2, §3] to describe modules over homomorphic images of these rings. The point here is that our construction of indecomposable $\Lambda$-modules given in $\S 2$ and $\S 3$ involves both $\Lambda$ and its normalization $\Gamma$, and so we need to describe how to deal with $\Gamma$ when passing to homomorphic images of $\Lambda$.

We use the commutative case of the following well-known result. (For the finitely generated case, which is all we need, see for example [CR1, Theorem 6.30].)

Lemma 11.1. Let $A$ be a quasi-Frobenius (i.e., artinian self-injective) ring with left socle $H$. Then every left $A$-module is the direct sum of a projective $A$-module and and an $\mathrm{A} / \mathrm{H}$-module.

Definition 11.2 (Klein rings). For any module $M$ over a ring $\Omega$, we let $\mu_{\Omega}(M)$ denote the minimal number of generators required by $M$.

We call the artinian local ring $(\Omega, \mathfrak{n}, k)$ a Klein ring if $\mu_{\Omega}(\mathfrak{n})=2, \mu_{\Omega}\left(\mathfrak{n}^{2}\right)=$ $1, \mathfrak{n}^{3}=0$, and every element of $\mathfrak{n}$ has square 0 . (See [KL1, Introduction, Theorem 2.10, and §5] for more about these rings.)

The next result reduces the description of modules over Klein rings to modules over homomorphic images of Dedekind-like rings.

Theorem 11.3. Suppose that $(\Omega, \mathfrak{n}, k)$ is a Klein ring; then the following hold.

(i) $\Omega$ is a quasi-Frobenius ring with simple socle $\mathfrak{n}^{2}$, and $k$ has characteristic 2 . 
(ii) $\Omega / \mathfrak{n}^{2}$ is a homomorphic image of a strictly split Dedekind-like ring $(\Lambda, \mathfrak{m}, k)$ which is $\mathfrak{m}$-adically complete; in fact, $\Omega / \mathfrak{n}^{2} \cong \Lambda / \mathfrak{m}^{2}$.

(iii) Every $\Omega$-module is the direct sum of a free module and an $\Omega / \mathfrak{n}^{2} \cong$ $\Lambda / \mathfrak{m}^{2}$-module.

Proof. (i) Since $\mathfrak{n}^{3}=0$ we have $\mathfrak{n}^{2} \subseteq \operatorname{soc} \Omega$. We can write $\mathfrak{n}=(x, y)$. From the definition, we conclude that $x^{2}=y^{2}=0$, but $x y \neq 0$ since $\mu_{\Omega}\left(\mathfrak{n}^{2}\right)=1$, so in fact $\mathfrak{n}^{2}=(x y)$. Thus $\mathfrak{n}^{2}$ is a simple submodule of $\operatorname{soc} \Omega$. To see that $\mathfrak{n}^{2}$ is the entire socle of $\Omega$ suppose that $(a x+b y) \mathfrak{n}=0$. Then $\mathfrak{n}=(x, y)$ shows that $a x y=0=b x y$, and hence both $a$ and $b$ are nonunits, that is, elements of $\mathfrak{n}$. Therefore, $a x+b y \in \mathfrak{n}^{2}$, as desired.

Also from the definition of Klein ring, it follows that $0=(x+y)^{2}=$ $x^{2}+2 x y+y^{2}=2 x y$, from which we conclude that $2 \in \mathfrak{n}$, and hence the residue field $k$ has characteristic 2 . Since $\Omega$ is artinian and local with simple socle, $\Omega$ is quasi-Frobenius [F, Theorem 3.1].

(ii) $\Omega / \mathfrak{n}^{2}$ is a homomorphic image of a Dedekind-like ring $(\Lambda, \mathfrak{m}, k)$ of the claimed form, by [KL1, Proposition 3.4]. Say $\Omega / \mathfrak{n}^{2} \cong \Lambda / I$. Since the maximal ideal of $\Omega / \mathfrak{n}^{2}$ has square zero, we have $I \subseteq \mathfrak{m}^{2}$. To see that equality holds it suffices to show that $\Omega / \mathfrak{n}^{2}$ and $\Lambda / \mathfrak{m}^{2}$ both have composition length 3 . This holds because $\mu_{\Omega}(\mathfrak{n})$ and $\mu_{\Lambda}(\mathfrak{m})$ both equal 2 , and hence each maximal ideal modulo its square has $k$-dimension 2 .

(iii) This follows from statements (i) and (ii), together with Lemma 11.1 and the fact that projective modules over local rings are free.

The rest of this section deals with modules over homomorphic images of unsplit or strictly split Dedekind-like rings.

Lemma 11.4. Let $I \neq 0$ be an ideal of an unsplit or strictly split Dedekindlike ring $(\Lambda, \mathfrak{m}, k)$ with normalization $\Gamma$. Then exactly one of the following holds.

(i) $\mu_{\Lambda}(I)=1, I$ contains regular elements of $\Lambda$, and $\Lambda(\Gamma I) / I \cong k$.

(ii) $\mu_{\Lambda}(I)=2$, I contains regular elements, and $\Gamma I=I$.

(iii) $\mu_{\Lambda}(I)=1, I$ contains no regular elements of $\Lambda$, and $\Gamma I=I$.

The module $\Lambda(\Lambda / I)$ has finite length in situations (i) and (ii), but not (iii). In situation $(\mathrm{i}),(\Gamma I) / I=\operatorname{soc}_{\Lambda}(\Lambda / I)$.

Proof. Recall that $\Gamma / \mathfrak{m}$ is a 2 -dimensional $k$-vector space in both the unsplit and strictly split cases [Notation 1.1]. It follows from Nakayama's lemma that $\mu_{\Lambda}\left({ }_{\Lambda} \Gamma\right)=2$. Again by Nakayama's lemma, we have

$$
0 \neq I /(\mathfrak{m} I) \subseteq(\Gamma I) /(\mathfrak{m} I) .
$$

Note that the $\Lambda$-modules on both sides of the inclusion in (11.4.1) are annihilated by the ideal $\mathfrak{m}$ and are therefore $k$-vector spaces.

Consider the case that $I$ contains regular elements of $\Lambda$. These are also regular elements of the principal ideal ring $\Gamma$, and therefore $(\Gamma I) /(\mathfrak{m} I) \cong$ 
$\Gamma / \mathfrak{m}$ as $\Lambda$-modules, hence has dimension 2 as a $k$-vector space. Suppose $\Gamma I \neq I$. Then the inclusion in (11.4.1) is strict. Since the right-hand side has $k$-dimension $2, I /(\mathfrak{m} I)$ therefore has $k$-dimension 1 , and therefore, by Nakayama's lemma, $\mu_{\Lambda}(I)=1$. Moreover, these dimensions show that $(\Gamma I) / I$ has $k$-dimension 1 as claimed. Thus all of the assertions in situation (i) hold in this case. On the other hand, suppose that $\Gamma I=I$. Then the inclusion in (11.4.1) becomes equality, showing that $I /(\mathfrak{m} I)$ has $k$-dimension 2 , and hence $\mu_{\Lambda}(I)=2$. Thus situation (ii) holds.

This leaves the case that $I$ consists of zero divisors, and hence the Dedekindlike ring $\Lambda$ is strictly split. It follows that $I$ is contained in one of the two coordinate rings of $\Gamma$. In this situation situation (iii) is easily seen to hold, and $\Lambda / I$ does not have finite length.

Now we claim that, in situation (i),

$$
\operatorname{soc}_{\Lambda}(\Lambda / I)=(\Gamma I) / I \text {. }
$$

We already have the inclusion $(\supseteq)$ by (i). Let $\operatorname{soc}(\Lambda / I)=Y / I$. Since $\mathfrak{m}$ annihilates the simple $\Lambda$-module, we have $\mathfrak{m} Y \subseteq I$. Note that the lefthand side is a $\Gamma$-module while the right-hand side is not (since $\Gamma I \neq I$ in situation (i)). Therefore strict inequality holds. Moreover $\mathfrak{m} I$ is the unique maximal $\Lambda$-submodule of $I$, since $I$ is principal and generated by a regular element. It follows from these two observations that $\mathfrak{m} Y \subseteq \mathfrak{m} I$. Substituting $\mathfrak{m}=\pi \Gamma$, and canceling the regular element $\pi$ then shows that $Y \subseteq \Gamma Y \subseteq \Gamma I$, completing the proof of the claim.

The finite length assertion about situations (i) and (ii) follows from the facts that $\Lambda$ has Krull dimension 1 and $I$ contains regular elements.

Definition 11.5. An AVR - artinian valuation ring - is an artinian local principal ideal ring. These are precisely the artinian rings whose ideals are totally ordered by inclusion. Note that a field is an AVR but (by our convention in Notation 1.1) not a DVR.

Lemma 11.6. Let $(\Lambda, \mathfrak{m}, k)$ be an unsplit or strictly split Dedekind-like ring with normalization $\Gamma$, and let $I$ be an ideal of $\Gamma$ such that $0 \neq I \subseteq \mathfrak{m}$. Then $\Lambda / I$ is the pullback of the following commutative square analogous to (1.1.1).

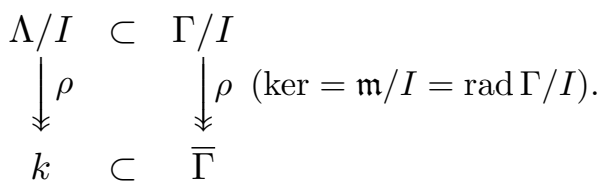

Moreover, $\Gamma / I$ is an AVR or the direct sum of two AVRs or the direct sum of one DVR and one AVR.

Proof. The fact that $\Lambda / I$ is the pullback of (11.6.1) is an immediate consequence of the fact that $\Lambda$ is the pullback of (1.1.1). The statements about 
$\Gamma / I$ are immediate consequences of the fact that $\Gamma$ is either a DVR or the direct sum of two DVRs.

The next theorem reduces the description of modules over homomorphic images of the Dedekind-like rings in this paper to the situation in diagram (11.6.1).

Theorem 11.7. Let $I$ be an ideal of an unsplit or strictly split Dedekindlike ring $(\Lambda, \mathfrak{m}, k)$ with normalization $\Gamma$, and suppose that $0 \neq I \subseteq \mathfrak{m}$. Then exactly one of the following holds.

(i) $I \neq \Gamma I$. Then every $\Lambda / I$-module is the direct sum of a free $\Lambda / I$-module and $a \Lambda /(\Gamma I)$-module, and $\Lambda /(\Gamma I)$ is a pullback as in (11.6.1).

(ii) $I=\Gamma I$. Then $\Lambda / I$ is the pullback of commutative square (11.6.1).

Proof. (i) By Lemma 11.4, the ring $\Lambda / I$ is an artinian ring with simple socle $(\Gamma I) / I$ and (obviously) has no nontrivial idempotents. Therefore $\Lambda / I$ is quasi-Frobenius [F, Theorem 3.1]. The desired decomposition of $\Lambda$-modules therefore follows from Lemma 11.1.

(ii) By hypothesis $I$ is an ideal of $\Gamma$, and so Lemma 11.6 applies.

It now suffices to describe all indecomposable finitely generated $\Lambda / I$ modules in the situation of diagram (11.6.1), where $0 \neq I \subseteq \mathfrak{m}$ and $I$ is an ideal of $\Gamma$. Our structure theorems in $\S 2$ and $\S 3$ all construct $\Lambda$-modules from $\Gamma$-modules, using standard diagrams. The key to moving between $\Lambda$ and $\Gamma$ is given in the next lemma, and the final answer is given in Theorem 11.9.

Lemma 11.8. Let $(\Lambda, \mathfrak{m}, k)$ be an unsplit or strictly split Dedekind-like ring with normalization $\Gamma$, and let $I$ be an ideal of $\Gamma$ such that $0 \neq I \subseteq \mathfrak{m}$. Let $M=M(\mathcal{D})$ be any finitely generated indecomposable $\Lambda$-module constructed from a diagram $\mathcal{D}$ and $a \Gamma$-module $X$, as in $\S 2$ and $\S 3$.

Then $I M=0$ (i.e., $M$ is a $\Lambda / I$-module) if and only if $I X=0$ (i.e., $X$ is a $\Gamma / I$-module).

Proof. We have $M=S(\mathcal{D}) / K(\mathcal{D})$ by (2.2.11) or (3.2.9) respectively, according to whether $\Lambda$ is unsplit or strictly split. Moreover, as shown in Subsection 9.6 and Theorem 6.6, the natural surjection $\phi: S=S(\mathcal{D}) \rightarrow M$ is a separated cover of $M$ in both cases; and $X=\Gamma S$ (in the unsplit case, by (9.1.1) and the fact that $A$ is invertible, in the strictly split case, by (6.4.3) and the fact that $A_{1}$ and $A_{2}$ are invertible). It therefore suffices to prove the following more general result:

(11.8.1) Let $\phi: S \rightarrow M$ be a separated cover of a $\Lambda$-module, and let $X=\Gamma S$ be any $\Gamma$-module generated by $S$. Then $I M=0$ if and only if $I X=0$.

To prove the nontrivial "only if" statement, supose that $I M=0$. Then $I S \subseteq \operatorname{ker} \phi$. Since $S$ is a $\Lambda$-submodule of some $\Gamma$-module, it follows that $I S$ 
is a $\Gamma$-submodule of ker $\phi$. Since $\phi$ is a separated cover, it follows that $I S=0$ [Lemma 4.9]. Since $I$ is an ideal of $\Gamma$ we therefore have $I X=I \Gamma S=I S=0$ as claimed.

Theorem 11.9. Let $(\Lambda, \mathfrak{m}, k)$ be an unsplit or strictly split Dedekind-like ring with normalization $\Gamma$, and $I$ an ideal of $\Gamma$ such that $0 \neq I \subseteq \mathfrak{m}$. Let $\mathcal{D}$ be a standard diagram, as in $\S 2$ or $\S 3$, constructed from some $\Gamma$-module $X$.

Then $M(\mathcal{D})$ is a $\Lambda / I$-module if and only if:

(i) If $\Lambda$ is unsplit, then all length-labels in $\mathcal{D}$ are less than or equal to the length of the AVR $\Gamma / I$ (as a $\Gamma / I$-module).

(ii) If $\Lambda$ is strictly split (so that $\Gamma=\Gamma_{1} \oplus \Gamma_{2}$, and $I=I_{1} \oplus I_{2}$, with each $\left.I_{\nu} \subset \Gamma_{\nu}\right)$, then all $\Gamma_{\nu}$-length labels $(\nu=1,2)$ in $\mathcal{D}$ are less than or equal to the length of the DVR or AVR $\Gamma_{\nu} / I_{\nu}$.

Proof. As shown in Lemma 11.8, we have $I M=0$ if and only if $I X=0$. Thus the question reduces to finding when $I X=0$.

Suppose first that $\Lambda$ is unsplit, hence $\Gamma$ is a DVR. Then $\Gamma / I$ is an AVR, and hence $X$ is a direct sum of uniserial $\Gamma$-modules whose length is at most the length of $\Gamma / I$. In other words, all length labels in $\mathcal{D}$ are at most the length of $\Gamma / I$, as claimed.

Suppose next that $\Lambda$ is strictly split. Then $\Gamma=\Gamma_{1} \oplus \Gamma_{2}$ where each $\Gamma_{\nu}$ is a DVR, and $I=I_{1} \oplus I_{2}$ where each $I_{\nu}$ is an ideal of $\Gamma_{\nu}$ contained in the maximal ideal of $\Gamma_{\nu}$. In this situation $\Gamma_{i} / I_{i}$ is either an AVR (if $I_{\nu} \neq 0$ ) or a DVR (if $I_{\nu}=0$ ). The rest of the proof is exactly as in the unsplit case.

Remark 11.10. It is possible to know that a commutative complete local ring $(\Upsilon, \mathfrak{p}, k)$ is a homomorphic image of a complete Dedekind-like ring $(\Lambda, \mathfrak{m}, k)$ without knowing $\Lambda$ and its normalization $\Gamma$ in advance. For example, $\Upsilon$ might be equal $\Omega / \mathfrak{n}^{2}$ where $\Omega$ is a Klein ring with radical $\mathfrak{n}$ [Theorem 11.3].

Assume that $\Upsilon$ is known to be a homomorphic image of a complete Dedekind-like (local) ring. We may assume that the socle of $\Upsilon$ has $k$ dimension 2 (for if it equals 1 , we apply the reduction in the supplementary statement of Theorem 11.7). Thus we are now in the situation shown in (11.6.1): $I$ is an ideal of $\Gamma$ contained in $\mathfrak{m}$. In this situation, one must know something about $\Gamma$ in order to obtain the structure of indecomposable $\Lambda$ modules from Theorem 11.9. A careful reading of the results in Sections 2 and 3 to which Theorem 11.9 refers shows that it suffices to know $\Gamma / I$, rather than $\Gamma$ itself.

We remark that the proofs of [KL1, Propositions 3.4 and 3.6] give constructions of $\Lambda$ and $\Gamma$ in the strictly split and unsplit cases, respectively, and this can presumably be used to find the structure of $\Gamma / I$. 


\section{Examples.}

This section gives several naturally occurring examples of unsplit and strictly split Dedekind-like rings, both complete and not complete. We repeatedly use the fact that tensoring a pullback diagram with a flat module yields a pullback diagram (use the idea in e.g. [K, Proposition 2.10] or [L3, Lemma 6.1]). We also use Lemma 2.21 of [KL1], which reads: If $(\Lambda, \mathfrak{m}, k)$ is an unsplit or strictly split Dedekind-like ring with normalization $\Gamma$, then the $\mathfrak{m}$-adic completion $(\hat{\Lambda}, \hat{\mathfrak{m}}, k)$ is, respectively, an unsplit or strictly split Dedekind-like ring with normalization $\hat{\Gamma}$, and $\operatorname{rad} \hat{\Gamma}=\hat{\mathfrak{m}}$.

Examples 12.1 (Simplest examples). The ring $\mathbb{R}+X \mathbb{C}[[X]]$ of formal power series over the complex numbers, with real constant term is a complete unsplit Dedekind-like ring [KL1, Examples 2.18].

The ring $k[[X, Y]] /(X \cdot Y)$, with $k$ any field, is complete strictly split Dedekind-like, and so is $\hat{\mathbb{Z}}_{p}[[X]] /(p \cdot X)$, where $p$ is any prime number and $\hat{\mathbb{Z}}_{p}$ is the $p$-adic completion of the integers [KL1, Examples 2.17].

Example 12.2. Let $\Lambda=\mathbb{Z} G$, the integral group ring of any finite cyclic group of squarefree order. Then, for every maximal ideal $\mathfrak{m}$ of $\Lambda$, the $\mathfrak{m}$ localization $\Lambda_{\mathfrak{m}}$ is either strictly split Dedekind-like or a DVR. (See [L2], where slightly different terminology is used.) Hence by [KL1, Lemma 2.21], this is also true of the $\mathfrak{m}$-adic completion $\hat{\Lambda}_{\mathfrak{m}}$.

Example 12.3. (In the language of algebraic geometry:) The coordinate ring of the union of the two lines $X=0$ and $Y=0$ over the field $k$ is the prototypical strictly split Dedekind-like ring $k[X, Y] /(X Y)$. More generally, let $\Lambda$ be the coordinate ring of the union of finitely many distinct lines, where each pair of lines intersect in a point, but no three of the lines intersect in a point. Then each of the localizations of $\Lambda$ at one of the maximal ideals corresponding to one of these points of intersection is a strictly split Dedekind-like ring. By [KL1, Lemma 2.21], the same is true of the completions.

Example 12.4 (Quadratic orders). Consider the quadratic order $\Lambda=$ $\mathbb{Z}[\sqrt{n}]$ for some square-free integer $n$. We show that the p-adic completion $\hat{\Lambda}_{p}$ of $\Lambda$ is strictly split Dedekind-like, unsplit Dedekind-like, or a DVR for every rational prime $p$.

It is well-known ([We, Theorem 6-1-1], for example) that $\Lambda$ is a Dedekind domain if and only if $n$ is congruent to 2 or 3 modulo 4 . Thus, we can suppose that $n$ is congruent to 1 modulo 4 . In this situation it is well-known that $\Gamma=\mathbb{Z}[(1+\sqrt{n}) / 2]$ is the normalization of $\Lambda$. (See, for example, [We, Theorem 6-1-1].) If $p$ is an odd prime, the $p$-adic completions of $\Lambda$ and $\Gamma$ are obviously the same, and hence $\hat{\Lambda}_{p}$ is a DVR, so we need only consider the 2 -adic completion $\hat{\Lambda}_{2}$. 
Let $C=2 \Gamma$; clearly $C \subseteq \Lambda$, so that $C$ is contained in the conductor of $\Gamma$ into $\Lambda$. By [We, Theorem 6-2-1], the ideal $C$ is maximal in $\Gamma$ if $n$ is congruent to 5 modulo 8 , while $C$ is the product of two distinct maximal ideals in $\Gamma$ if $n$ is congruent to 1 modulo 8. We consider these two cases individually.

Consider the case $n \equiv 5(\bmod 8)$. Here, the residue ring $\Gamma / C$ is a field with exactly four elements, and $\Lambda / C$ is a proper subring of $\Gamma / C$, so that $k=\Lambda / C$ is a field with exactly two elements. Therefore, $C$ is also a maximal ideal of $\Lambda$ and so is the conductor from $\Gamma$ into $\Lambda$. Moreover, the rings $\Lambda$ and $\Gamma$ fit into a conductor square as in (1.1.1) (except for the kernels of the vertical maps being the radical), in which $F=\Gamma / C$ is a (separable) quadratic field extension of $k$. Since tensoring a pullback diagram with a flat module again yields a pullback diagram, tensoring this diagram with $\hat{\mathbb{Z}}_{2}$ shows that $\hat{\Lambda}_{2}$ is unsplit Dedekind-like. In fact, the localization $\Lambda_{2}$ is also unsplit Dedekindlike.

Consider the case $n \equiv 1(\bmod 8)$. Here the residue $\operatorname{ring} \Gamma / C$ is the product of two fields each with exactly two elements, and $\Lambda / C$ is a proper subring of $\Gamma / C$. Again it follows that $k=\Lambda / C$ is a field with exactly two elements, so that $C$ is a maximal ideal of $\Lambda$ and is the conductor from $\Gamma$ into $\Lambda$. Moreover, the rings $\Lambda$ and $\Gamma$ fit into a conductor square as in (1.1.1) (except for the kernels of the vertical maps being the radical), in which the inclusion in the bottom row can be taken to be the diagonal inclusion of $k$ into $\Gamma / C \cong k \times k$. As before, tensoring with $\hat{\mathbb{Z}}_{2}$ shows that $\hat{\Lambda}_{2}$ is strictly split Dedekind-like. Here the localization $\Lambda_{2}$ is never strictly split Dedekind-like, because it is an integral domain. (However it is nonstrictly split Dedekind-like, as defined in [KL1, Definitions 2.5].)

Example 12.5. Let $\Lambda$ be a subring of square-free index (say, $n$ ) in $\Gamma=\mathbb{Z}^{s}$ (direct product of $s$ copies of the integers). Then we claim that, for every maximal ideal $\mathfrak{m}$ of $\Lambda$, the localization $\Lambda_{\mathfrak{m}}$ is a strictly split Dedekind-like ring or a DVR. (Hence, by [KL1, Lemma 2.21], the same is true of the $\mathfrak{m}$-adic completion $\hat{\Lambda}_{\mathfrak{m}}$.) In fact, $\Lambda_{\mathfrak{m}}$ is strictly split Dedekind-like precisely for the finite number of $\mathfrak{m}$ that contain a prime factor of $n$.

We may assume that $n>1$. Since $|\Gamma / \Lambda|=n$ we have

$$
\Gamma n \subset \Lambda \subset \Gamma \text {. }
$$

Choose a maximal ideal $\mathfrak{m}$ of $\Lambda$. We want to show that $\Lambda_{\mathfrak{m}}$ is strictly split Dedekind-like with normalization $\Gamma_{\mathfrak{m}}$, or else is a DVR.

If $\mathfrak{m} \nsupseteq \Gamma n$, then some element of $\Gamma n$ becomes a unit in $\Lambda_{\mathfrak{m}}$, and we have $\Lambda_{\mathfrak{m}}=\Gamma_{\mathfrak{m}}$, a local principal ideal domain, equivalently, a DVR. Thus we suppose, from now on, that $\mathfrak{m} \supseteq \Gamma n$. There are only finitely many such $\mathfrak{m}$ since $\Gamma / \Gamma n$ is a finite ring.

Since $n \in \mathfrak{m}$, the prime ideal $\mathfrak{m}$ contains some prime number $p$ that divides $n$. Since localizing at $\mathfrak{m}$ can be done by first localizing at $p$, we may replace 
the three terms in (12.5.1) by their $p$-localizations. The effect of this is that, after a change of notation, we may assume that $n=p$, a prime number. Moreover the original aditive group $\Gamma / \Lambda$ of square-free order $n$ has now been replaced by its $p$-localization, hence is a group of order $p$. We now have (after our change of notation) that $\Lambda$ is the pullback of the following diagram.

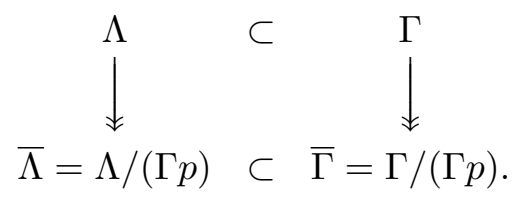

Note that $\bar{\Lambda}$ and $\bar{\Gamma}$ are finite rings. Now localize the whole diagram at $\mathfrak{m}$. Then $\Lambda_{\mathfrak{m}}$ remains the pullback of the localized diagram. Thus, after a further change of notation, we have that $\Lambda$ is a local ring. We now prove that $\Lambda$ is strictly split Dedekind-like with normalization $\Gamma$.

Think of the original rings $\Lambda$ and $\Gamma$ as module-finite $\mathbb{Z}$-algebras. Then our first localization - at $p$ - converted $\Lambda$ and $\Gamma$ to module-finite $\mathbb{Z}_{p^{-}}$-algebras. In particular, it converted $\mathbb{Z}^{s}$ (the original $\Gamma$ ) to $\mathbb{Z}_{p}^{s}$. The effect of the further localization at $\mathfrak{m}$ on each coordinate ring $\mathbb{Z}_{p}$ is to either leave it unchanged or replace it with 0 . Thus we now have $\Gamma=\mathbb{Z}_{p}^{t}$ for some $t \leq s$. Therefore $\bar{\Gamma}=\overline{\mathbb{Z}}_{p}^{t}$, the direct product of $t$ copies of the field of $p$ elements.

Now consider the finite, therefore artinian ring $\bar{\Lambda}$. Since $\bar{\Lambda} \subseteq \bar{\Gamma}, \bar{\Lambda}$ has radical zero and is therefore a direct product of fields. On the other hand $\bar{\Lambda}$ is a local ring, because the local ring $\Lambda$ maps onto it. Therefore $\bar{\Lambda}$ is a field (of characteristic $p$ ). Since each of the coordinate rings $\overline{\mathbb{Z}}_{p}$ of $\bar{\Gamma}$ is a $\bar{\Lambda}$-vector space, we see that $\bar{\Lambda}=\overline{\mathbb{Z}}_{p}$.

The abelian group $\bar{\Gamma} / \bar{\Lambda} \cong \Gamma / \Lambda$ has order $p$. On the other hand its order is $|\bar{\Gamma}| /|\bar{\Lambda}|=p^{t} / p=p^{t-1}$. Therefore $t=2$. We now have that $\Gamma=\mathbb{Z}_{p}^{2}$, $\bar{\Gamma}=\overline{\mathbb{Z}}_{p}^{2}$, and $\bar{\Lambda}=\overline{\mathbb{Z}}_{p}$. Therefore the local ring $\left(\Lambda, \Gamma p, \overline{\mathbb{Z}}_{p}\right)$ is strictly split Dedekind-like, as desired.

Example 12.6. It is easy to see, by continuing the reasoning in the previous proof, that the rings $\Lambda$ in Example 12.5 can be explicitly constructed as follows.

Choose any square-free positive integer $n=p_{1} p_{2} \ldots p_{u}>1$ and any positive integer $s>1$. For each prime $p_{\nu}$ choose a pair of coordinate rings, say coordinates $i_{\nu} \neq j_{\nu}$ of $\mathbb{Z}^{s}$. Let $f_{\nu}, g_{\nu}$ be the natural map of coordinate rings $i_{\nu}, j_{\nu}$ respectively, onto $\overline{\mathbb{Z}}_{p_{\nu}}$. Then let

$$
\Lambda=\left\{\left(x_{1}, \ldots, x_{s}\right) \in \mathbb{Z}^{s} \mid(\forall \nu) f_{\nu}\left(x_{i_{\nu}}\right)=g_{\nu}\left(x_{j_{\nu}}\right)\right\} .
$$

For some amusing module-theoretic parlor tricks over these rings - that is, many examples of nonuniqueness of direct-sum decompositions of finitely generated $\Lambda$-modules — see $[\mathbf{L} \mathbf{4}]$. 


\section{Terminological index.}

\section{Item}

associated

4-tuple $f(p)$ of matrix pair $p$

$\Gamma$-module (split case)

$\Gamma$-module (unsplit case)

$\Lambda$-module (split case)

$\Lambda$-module (unsplit case)

AVR (artinian valuation ring)

block size, in

diagram (split case)

diagram (unsplit case)

matrix 4-tuple

matrix pair

blocking matrix

split case (block cycle 4-tuple)

split case (module)

unsplit case (matrix pair)

unsplit case (module)

concatenation (of sequences)

conjugate

element, matrix, or 4-tuple

self-conjugate 4 -tuple

corresponding

column (split case)

column (unsplit case)

summand (split case)

summand (unsplit case)

cycle

Dedekind-like ring

direct sum of matrix 4-tuples

display operation

$\begin{array}{ll}\text { (split case) } & 6.5 \\ \text { (unsplit case) } & 5.9 \\ \text { DVR } & 1.1 \\ \text { efficient diagram } & \end{array}$

efficient diagram

split case

3.7

unsplit case

2.10

glue

split case

3.2

unsplit case

2.2

11.2

Klein ring

Krull-Schmidt theorem

(Dedekind-like rings)

label sequence, in

diagram (split case)

diagram (unsplit case)

matrix 4-tuple (deleted cycle)

matrix 4-tuple (block cycle)

matrix pair (no blocking matrix)

1.3

8.7

matrix pair (with blocking matrix) 8.10

length label, in

\section{Item}

diagram (split case)

diagram (unsplit case)

matrix (split case)

matrix (unsplit case)

local ring

matrix

4-tuple

canonical forms (split case)

canonical forms (unsplit case)

operations theorem (split case)

operations theorem (unsplit case)

pair

setup (split case)

setup (unsplit case)

$\mathcal{X}$ triangular form

mirror image (permutation)

moving $U$

split case

unsplit case

package (matrix 4-tuple)

reduce (top or bottom)

repetition-free sequence

separated cover

almost functorial

definition

earlier work

uniqueness

separated module

connotation of words

definition

examples of nonseparated

simultaneous cyclic permutations

standard

copy of $F$

copy of $k$

diagram (split case)

diagram (unsplit case)

generator

image (of element of $F$ )

image (of element of $k$ )

sweeping-similarity operations

$k-k$ (split case)

$k-F$ (unsplit case)

tame

Theorem 90 for matrices

tile

basic

exceptional

unit forward rotation

\section{Section}

3.2

2.2

6.4

5.7

1.1

6.4

7.2

$8.7,8.10$

6.7

5.11

5.7

6.3

5.6

5.12

2.3, 8.6

3.6

2.9

8.1

2.2

4.12

4.6

4.8(i)

4.13

4.17

4.3

4.16

3.4

$2.1,5.6$

$3.1,6.3$

3.3

$2.4,2.6$

1.1

5.6

6.3

6.7

5.11

1 ( 91$)$

8.11

2.1, 8.6 


\section{References}

[Ba] C.S. Ballantine, Products of complic cosquares and pseudo-involutory matrices, Lin. and Multilin. Alg., 8 (1979), 73-78, MR 0i:15017, Zbl 417.15011.

[CR1] C.W. Curtis and I. Reiner, Methods of Representation Theory, with Applications to Finite Groups and Associative Algebras, Volume 1, Wiley-Interscience, New York, 1981, MR 82i:20001, Zbl 469.20001.

[F] K. Fuller, On indecomposable injective modules over artinian rings, Pacific J. Math., 29 (1969), 115-135, MR 40 \#186, Zbl 182.05702.

[K] L. Klingler, Modules over the integral group ring of a nonabelian group of order pq, Mem. Amer. Math. Soc., 59 (1986), MR 7g:16015, Zbl 598.16014.

[KL0] L. Klingler and L.S. Levy, Sweeping-similarity of matrices, Lin. Alg. Appl., 75 (1986), 67-104, MR 87k:15015, Zbl 585.15001.

[KL1] _ Representation type of commutative noetherian rings I: Local wildness, Pacific J. Math., 200 (2001), 345-386.

[KL3] - Representation type of commutative noetherian rings III: Global wildness, in preparation.

[KL4] - Representation type of commutative noetherian rings IV: Global tameness, in preparation.

[L] L.S. Levy, Modules over pullbacks and subdirect sums, J. Algebra, 70 (1981), 50-61, MR 82j:16052a, Zbl 508.16008.

[L1] Mixed modules over $Z G, G$ cyclic of prime order, and over related Dedekind pullbacks, J. Alg., 71 (1981), 62-114, MR 82j:16052b, Zbl 508.16009.

[L2] $\quad Z G_{n}$-modules, $G_{n}$ cyclic of square-free order n, J. Alg., 93 (1985), 354375, MR 87k:20017b, Zbl 596.13010.

[L3] - Modules over Dedekind-like rings, J. Alg., 93 (1985), 1-116, MR 87k:20017a, Zbl 564.13010.

[L4] Krull-Schmidt uniqueness fails dramatically over subrings of $\boldsymbol{Z} \oplus \cdots \oplus \boldsymbol{Z}$, Rocky Mountain J. Math., 13 (1983), 659-678, MR 85c:13008, Zbl 539.13005.

[LO] L.S. Levy and C.J. Odenthal, Krull-Schmidt theorems in dimension 1, Trans. Amer. Math. Soc., 348 (1996), 3391-3455, MR 96m:16006a, Zbl 858.16016

[NR] L.A. Nazarova and A.V. Roiter, Finitely generated modules over a dyad of two local rings and finite groups with an abelian normal divisor of Index $p$, Zap. Nauch. Sem. Leningrad Odtel. Mat. Inst. Steklov (LOM1), Izv. Akad. Nauk SSSR Ser mat., 33(1) (1969); English transl: USSR-Izv, 3(1) (1969), MR 41 \#5479, Zbl 207.04803.

[NRSB] L.A. Nazarova, A.V. Roiter, V.V. Sergeichuk and V.M. Bondarenko, Application of modules over a dyad for the classification of finite p-Groups that have an abelian subgroup of index $p$ and of pairs of mutually annihilating operators (Russian), Zap. Nauch. Sem. Leningrad Odtel. Mat. Inst. Steklov (LOM1), 28 (1972), 69-92; English transl: J. Soviet Math., 3 (1975), 636-653, MR 48 \#11288, Zbl 346.13005.

[We] E. Weiss, Algebraic Number Theory, Chelsea Publishing Company, New York, 1963, MR 28 \#3021, Zbl 115.03601.

Received September 9, 1999 and revised November 29, 2000. Levy's research was partially supported by an NSA grant. 
Mathematics Department

Florida AtLANTiC University

Boca Raton, Florida 33431-0991

E-mail address: klingler@fau.edu

Mathematics Department

UNIVERSITY OF WISCONSIN

MADISON, Wisconsin 53706-1388

E-mail address: levy@math.wisc.edu 